\title{
GLOBAL DESIGN OPTIMIZATION FOR AERODYNAMICS AND ROCKET PROPULSION COMPONENTS
}

\author{
Wei Shyy. Nilay Papila. Rajkumar Vaidyanathan \& Kevin Tucker ${ }^{*}$
}

Department of Aerospace Engineering, Mechanics and Engineering Science

University of Florida, Gainesville, FL

*NASA Marshall Space Flight Center, AL 


\begin{abstract}
Modem computational and experimental tools for aerodynamics and propulsion applications have matured to a stage where they can provide substantial insight into engineering processes involving fluid flows, and can be fruitfully utilized to help improve the design of practical devices. In particular, rapid and continuous development in aerospace engineering demands that new design concepts be regularly proposed to meet goals for increased performance, robustness and safety while concurrently decreasing cost. To date, the majority of the effort in design optimization of fluid dynamics has relied on gradient-based search algorithms. Global optimization methods can utilize the information collected from various sources and by different tools. These methods offer multi-criterion optimization, handle the existence of multiple design points and trade-offs via insight into the entire design space, can easily perform tasks in parallel, and are often effective in filtering the noise intrinsic to numerical and experimental data. However, a successful application of the global optimization method needs to address issues related to data requirements with an increase in the number of design variables, and methods for predicting the model performance. In this article, we review recent progress made in establishing suitable global optimization techniques employing neural network and polynomial-based response surface methodologies. Issues addressed include techniques for construction of the response surface, design of experiment techniques for supplying information in an economical manner, optimization procedures and multi-level techniques, and assessment of relative performance between polynomials and neural newworks. Examples drawn from wing aerodynamics, turbulent diffuser flows, gas-gas injectors, and supersonic turbines are employed to help demonstrate the issues involved in an engineering design context. Both the usefulness of the existing knowledge to aid current design practices and the need for future research are identitied.
\end{abstract}

Keywords: Global Optimization. Response Surtace Methodology, Design of Experiments. Neural Networks. 


\section{TABLE OF CONTENTS}

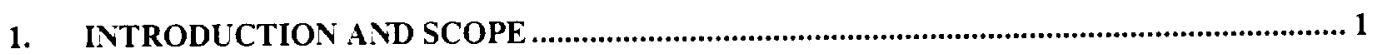

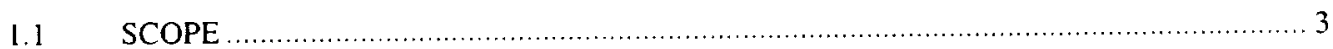

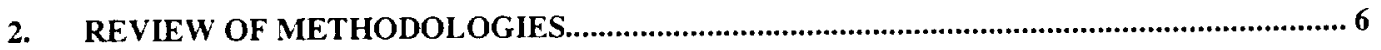

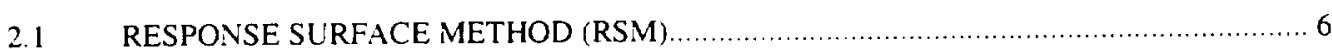

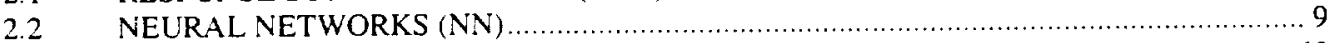

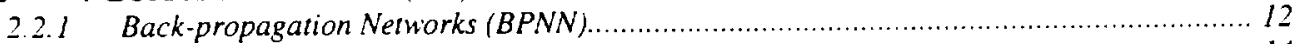

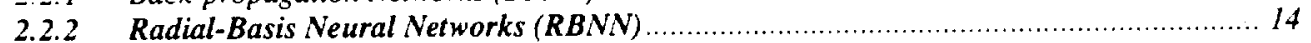

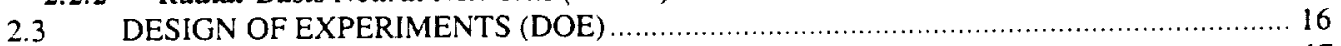

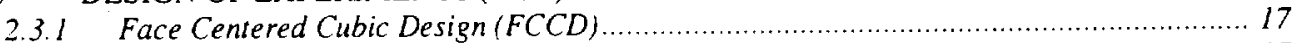

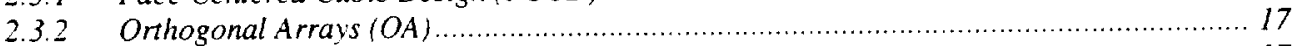

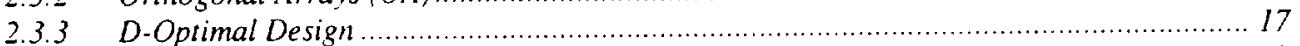

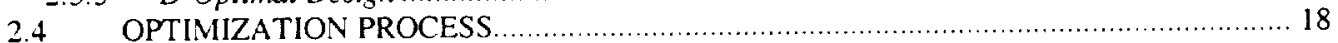

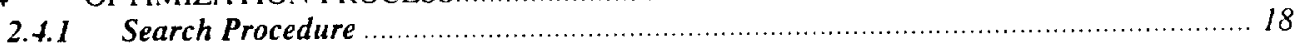

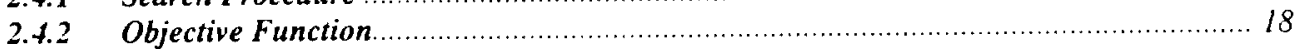

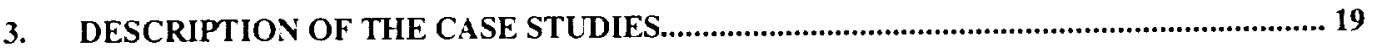

3.1 GAS-GAS INJECTOR ELEMENT FOR ROCKET PROPULSION ........................... 19

3.1.1 Shear Co-axial Injector Element.............................................................. 20

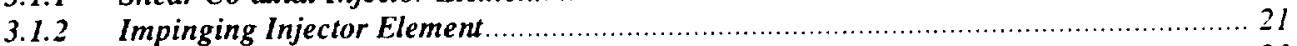

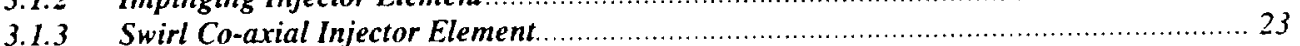

3.2 SUPERSONIC TURBINE FOR RELSABLE LAUNCH VEHICLES ........................ 26

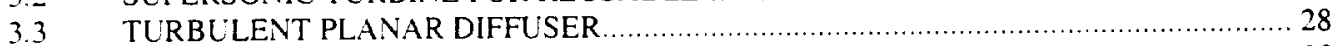

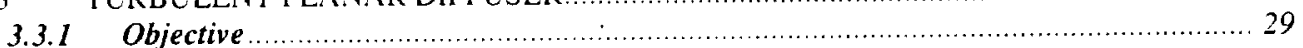

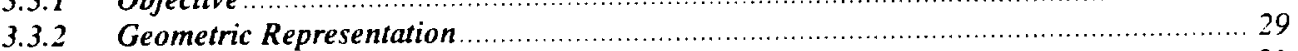

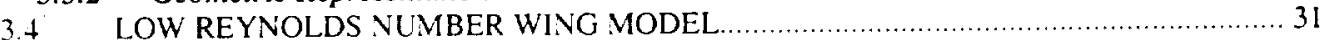

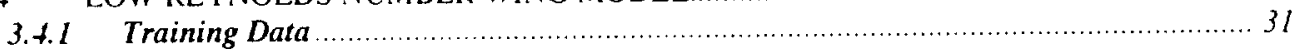

4. ASSESSMENT OF DATA PROCESSING AND OPTIMIZATION CAPABILITIES ............. 32

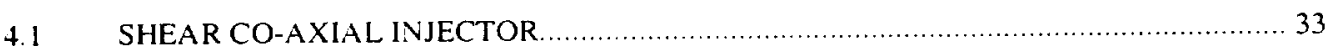

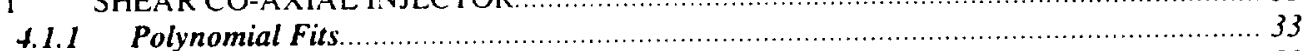

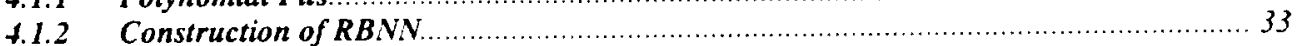

4.1.3 Evaluation of Polynomial and NN for Data Processing ........................................ 34

4.1.t Polynomial-based RSM for Design Optimization ............................................ 36

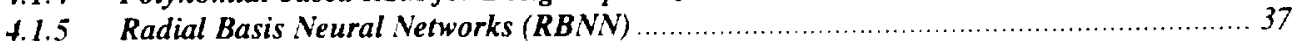

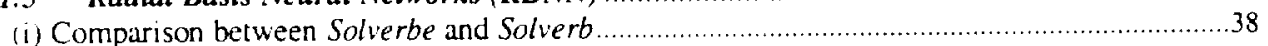

(ii) Comparison of RBNN Predictions with Polynomial Based RSM .........................................38

4.1.6 RBNN-Enhanced Polynomial Based Response Surface ..................................... 39

(i) Comparison of Fits with the Original Response Surfaces..................................................39

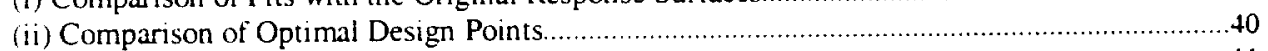

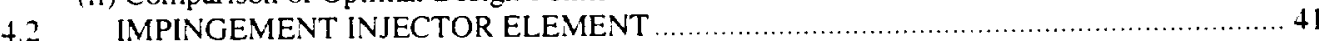

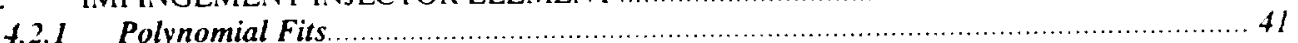

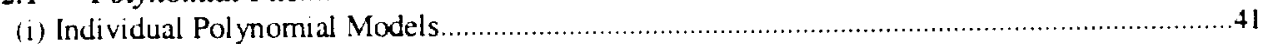

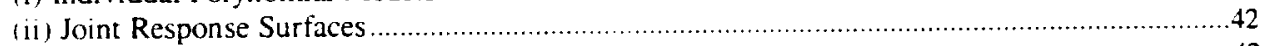

4.2.2 Optimization Results \& Discussion .......................................................... 42

(i) Effect of Each Variable on the Design Using Original Constraints \& Equal Weights ..............42

(ii) Emphasis on Lile \& Performance Issues Lsing Original Constraints \& Unequal Weights .......43 
(iii) Extraction of Last Performance \& Weight Increments (Modified Constraints \& Unequal Weights)

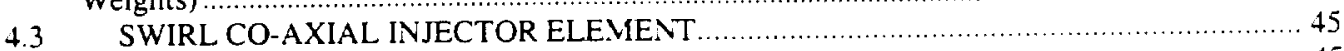

4.3.1 Effect Of Each Variable On Element Design ............................................... 45

4.3.2 Emphasis on Life and Performance Issues ................................................ 46

4.4 SUPERSONIC TURBINE FOR RELSA BLE LAUNCH VEHICLES ............................47

4.4.1 Polynomial-Based RSM Results for 1-, 2- and 3-Stage Turbines ........................... 47

4.4.2 Higher Order Polynomials and V.V-Based RSM for Single-Stage Turbine ................. 48

4.4.3 Orthogonal Arrays For 2-Stage Turbine ................................................... 50

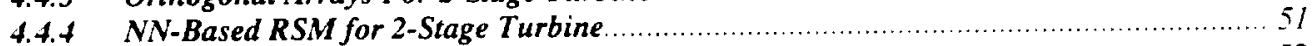

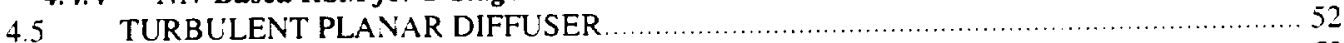

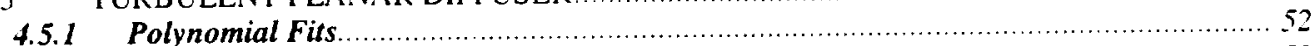

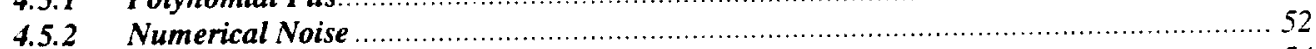

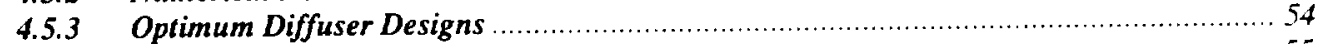

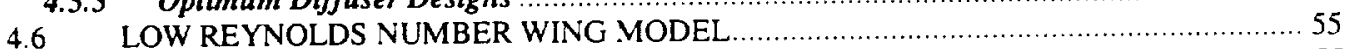

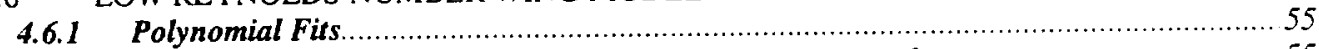

4.6.2 Comparison of Radial-Basis and Back-propagation Networks............................. 55

4.6.3 Comparison of Radial-Basis Neural Network and Polynomial-Based Techniques......... 57

5. CONCLUSION AND FUTURE DIRECTIONS.............................................................57

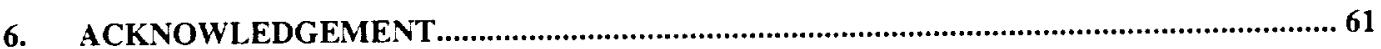

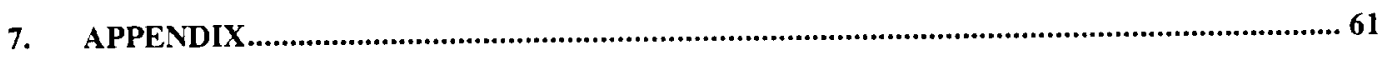

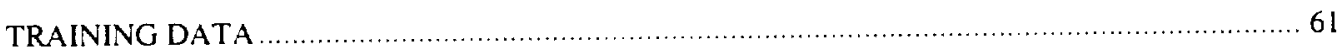

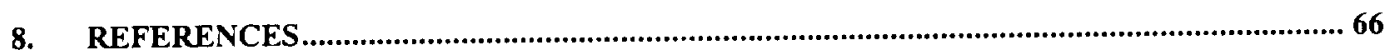

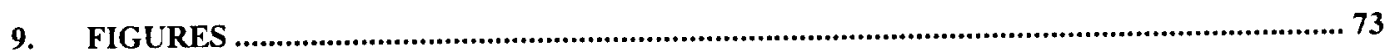

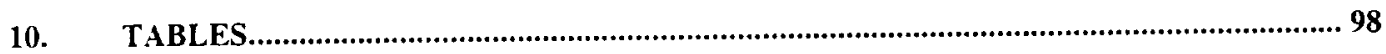




\title{
GLOBAL DESIGN OPTIMIZATION FOR AERODYNAMICS AND ROCKET PROPULSION COMPONENTS
}

\author{
Wei Shyy, Nilay Papila, Rajkumar Vaidyanathan and Kevin Tucker* \\ Department of Aerospace Engineering, Mechanics and Engineering Science University of Florida \\ NASA Marshall Space Flight Center, AL
}

\section{INTRODUCTION AND SCOPE}

Modern computational and experimental fluid dynamics tools have matured to a stage where they can provide substantial insight into engineering processes involving fluid flows. This can help analyze the fluid physics as well as improve the design of practical devices. In particular, rapid and continuous development in the technology of fluid machinery demands that new design concepts be regularly proposed to meet goals for increased performance, robustness and safety while concurrently decreasing cost.

Most aerospace system and component designs are conducted as open loop. feed-forward processes. For example, for rocket engines, currently, one design iteration for a given set of engine balance conditions takes up to several weeks with the blade geometry design sub-iteration phase taking several days each. The quest for an acceptable blade surface velocity distribution is accomplished with many ad hoc rules in what is really a manual trial-and-error process. A systematic approach capable of identifying optimum design and comparing possible trade-offs can significantly improve the productivity and shorten the design cycle

Objective and efficient evaluation of advanced designs can be facilitated by development and implementation of systematic optimization methods. To date, the majority of the effort in design optimization of fluid dynamics has relied on gradient-based search algorithms (Baysal and Eleshaky [1], Lambert et al.[27]. Reuther et al. [54]). These methods work iteratively through a sequence of local subproblems, which approximate objective and constraint functions for a sub-region of the design space, e.g., by linearization using computed sensitivities. Major challenges for these optimization approaches are the robust and speedy computation of sensitivity coefficients (Elbanna and Carlson [10], Dadone et al..66]).

Local optimization methods based on derivatives are commonly used in such engineering system design optimization problems (Sobieszczanski-Sobieski and Haftka [59|). On the other hand, global 
optimization techniques also have been commonly used for engineering design optimization problems especially for multidisciplinary ones. In its current practice, the global design optimization method involves three primary steps (Figure 1): (a) Generation of individual data sets within the design space; (b) Interpolation among these data sets via some continuous functional representation; and (c) Optimization of the objective function via a certain search strategy. Yet despite recent research advances, formal design optimization has yet to see practical use in real design scenarios. The reasons are five-fold:

(1) Engineering design, even within a single discipline, typically involves many parameters (and hence many degrees of freedom) rather than the handful demonstrated in most research papers. This renders unrestricted "brute force" search schemes too resource-intensive.

(2) The objective functions are likely to be multi-modal or discontinuous over the broad design space, rendering gradient search methods insufficient by themselves. Furthermore, the usual practice to combine multiple goals into a single quantitative objective function is too restrictive. Qualitative goals are often required to correctly characterize a problem. (E.g. maximizing a turbine blade's aerodynamic efficiency with a smooth, monotonic surface velœcity distribution. while spreading heat load as uniformly as possible.) Furthermore, these goals may have arisen from diverse disciplines and are usually treated sequentially by different groups

(3) It is inadequate to think of the final product of a design process as a mere geometry. A "design" really encompasses a whole set of operating, manufacturing and project level decisions.

(4) As the interaction between numerical simulation and physical test data becomes stronger, the future engineering knowledge base is likely to consist of all sorts of heterogeneous data sources including test data, experimental data, past product experiences, semi-empirical modeling, and high fidelity simulations. Some data are anecdotal; others cover only small "patches" of the physical domain but are still useful for "reality checks". A unified tramework needs to be constructed for representation, capturing and mining of all these data types so the response functions can be continuously improved.

With the above observations, global optimization methods are attractive because they have several advantages when compared to local gradient-based methods (Shyy et al. [581):

(1) They do not require calculation of the local sensitivity of each design variable,

(2) They can utilize the information collected from various sources and by different tools. 
(3) They offer multi-criterion optimization,

(4) They can handle the existence of multiple design points and trade-offs,

(5) They easily perform tasks in parallel, and

(6) They can often effectively filter the noise intrinsic to numerical and experimental data.

Among global approximation techniques, the Response Surface Methodology (RSM) has gained the most attention since it consists of a simple way of connecting codes from various disciplines (Sobieszczanski-Sobieski and Haftka [59]). The RSM is a collection of mathematical and statistical tools used in investigative experimentation by scientists and engineers (Bauer Jr. et al. [2]). The RSM approach replaces the objective and constraint functions by simple functions, often polynomials, which are fitted to the carefully selected points. Since RS.M can utilize information collected from various sources and by different tools, it can also offer multi-criterion optimization, handle the existence of multiple design selections and related trade-offs. and address the noises intrinsic to numerical and experimental data. A main advantage of RSM is its robustness and intelligibility. Robustness and the smoothness of approximations in noisy environments are achieved by performing extra analyses, compared to the number of regression coefficients. This is a distinct advantage over derivative-based search algorithms, which may encounter difficulties in the presence of spurious local optima (Madsen et al. [32]).

\subsection{SCOPE}

In this article, we first review the basic concepts and methodologies, then assess the current statue, via case studies, of the global optimization techniques. Particular attention is paid to two different techniques to generate information to construct the response surface (RS) namely; Neural Network (NN) and polynomial-based Response Surface Methodology. Veural Networks are models that contain many simple linear and non-linear elements operating in parallel and connected in patterns (Greenman [14]). Polynomial-based RSM models the system with polynomials of assumed order and unknown coefficients. The solution for the set of coefficients that best fits the training data is a linear least square problem, making it trivial compared to the solution for $\mathrm{NN}$, which involves a nonlinear training process. In this article, two neural network types, namely, Back-Propagation NN (BPNN) and Radial Basis NN (RBNN). are investigated. 
The BPNN consists of multi-layer networks with differentiable activation function. The BPNN is the most employed $\mathrm{NN}$ type in the optimization literature (Carpenter and Barthelemy [4], Faller and Schereck [11], Fan et al. [11], Greenman [14], Greenman, and Roth [15] \& [16], Huang et al. [20], Illi et al. [21], Kangas et al. [24], Lavretsky [28], Lawrence et al [29] \& [30], Madavan et al.[31], Maghami and Sparks [34] \& [35], Nikolaidis et al. [39], Norgaard et al. [40], Rai and Madavan [51], [52] \& [53], Ross et al. [55], Sparks and Maghami [60], Stepniewski and Jorgenson [61], and Stepniewski et al. [62]).

RBNN is a more recently developed multi layer network with a linear regression process that makes the mathematics simpler and computational costs lower (Orr [41], [42] \& [43]). RBN.V tends to have many more neurons than BPNN but can be configured faster for the same training data. The basic reason for this is that back-propagation neurons can have outputs over a large region of the input space, while radial-basis neurons respond to relatively small regions of the input space. Thus, larger input spaces require more radial-basis neurons for training. More detailed evaluation of RB.VN and BPNN will be given in the following sections.

Polynomial-based response surfaces and linear regression techniques have been originally developed to filter noise from experimental data. Sophisticated statistical tools are available for these purposes. One class of tools, design of experiments, is otten used to select points for training that minimize the effect of noise on the fitted polymomial. A second set of tools, analysis of variance, is routinely used to identify polymomial coefficients that are not well characterized by the data, and are therefore overly sensitive to noise. Analysis of variance helps avoid overtitting of the data, which otherwise would result in the mapping of the noise. On the other hand, neural networks are much more flexible in tunctional form, which means that they can be better suited to fit complex functions that are not easily approximated by polynomials. For example, when the physical system changes from one regime to another due to the presence of critical parameters, NN performs better than RSM. This advantage is particularly useful when there is very little numerical noise, and it is possible to obtain very accurate approximations to the underlying function (Papila et al.[49]). The relative strengths and weaknesses of NN and polynomial-based RSM are summarized in Table 1.

Table 2 summarizes the existing literature evaluating the relative performance of $N N$ and polynomial-based RSM approximation. For example, Carpenter and Barthelemy [4] used NN and 
polynomial-based approximations to develop RS for several test problems. It is demonstrated that two methods perform comparably based on the number of undetermined parameters. Rai and Madavan [51] investigated the feasibility of applying neural networks to the design of turbomachinery airfoils. Neural network approach is used for both function approximation and prediction. It is found that neural networks are quite efficient in both tasks. An aerodynamic design procedure that employs a strategy called parameter-based partitioning incorporating the advantages of both traditional RSM and NiNs to create a composite response surface is described by Rai and Madavan [52] \& [53]. It is shown that such method can handle design problems with higher dimensional problems than would be possible using NN alone. Nikolaidis et al. [39] used NNs and response surface polynomials to predict the performance characteristics of automotive joints using geometrical parameters. It is shown that both methods performed comparably. NN-based aerodynamic design procedure is applied to the redesign of a transonic turbine stage to improve its unsteady aerodynamic performance by Madavan et al. [31]. It is illustrated that using an optimization procedure combining the advantages of NN and polynomial-based RSM can be advantageous. Papila et al. [49] investigated the relative merits of polynomial-based RSM, RBNN and BPNN in handling different data characteristics. It is demonstrated that using RBNN rather than BPNN has certain advantages as data size increases. Also, it is shown that RBNN gives more accurate results than polynomila-based RSM as the nature of the experimental data becomes complex. Shyy et al. [57] have integrated neural network techniques and polynomial-based RSM to obtain improved optimization tools. In Rai and Madavan [53]. a composite NN \& polynomial-based RS methodology is applied for a transonic turbine and it is demonstrated that a systematic application of such method can enhance the effectiveness of the overall optimization process. In the study by Vaidyanathan et al. [71], the application of NN and polynomial-based RSM in preliminary design of two rocket engine components, gas-gas injector and supersonic turbine, with modest amounts of data are discussed and it is demonstrated that $\mathrm{NN}$ and polynomial-based approximations can perform comparably for modest data sizes.

In this article, we tocus on the recent efforts in developing, improving. and optimizing appropriate techniques for design optimization of airfoils and rocket engine components capable of being used in applications like Reusable Launch Vehicles. Some of the physical components used as case studies are low 
Re aerodynamics, 2-D turbulent planar diffuser, the injector and the supersonic turbine for rocket propulsion.

Specifically, the following issues are discussed:

(1) The capability of the NN and polynomial-based RSM for handling data with variable sizes and noise,

(2) The selection of NN configuration that is suitable for given design problems,

(3) The effect of the design parameters on the performance of the $\mathrm{NN}$,

(4) The effect of distribution of the data over the design space in the construction of the global model,

(5) The merit of employing a multi-level optimization strategy to perform the task adaptively and efficiently,

(6) Possible trade-offs between capacity design objectives and their impact on design selections.

\section{REVIEW OF METHODOLOGIES}

In response-surface-based global optimization, there are several key technical elements:

(1) Response surface with polynomials and statistical analysis

(2) Neural networks with BPNN and RBNN

(3) Design of experiments with face centered composite design (FCCD), orthogonal arrays (OA) and DOptimal designs

(4) Optimization procedure including the multilevel approach In the following, we review these elements in sequence.

\subsection{RESPONSE SURFACE METHOD (RSM)}

The approach of RSM is to perform a series of experiments, based on numerical analyses or physical experiments, for a prescribed set of design points, and to construct a global approximation of the measured quantity over the design space (Figure 1). The polynomial-based RSM, used in all the case studies referred to, constructs polynomials of assumed order and unknown coefficients based on regression analysis. The number of coefficients to be evaluated depends on the order of polynomial and the number of design parameters involved. For instance, a second-order polynomial of $N$ design variables has $(N+1)(N+2) / 2 !)$ coefficients. A cubic model has $(N+1)(N+2)(N+3) /(3 !)$ coefficients. In this article, the 
polynomial approximations are constructed by standard least square regression using JMP [23], a statistical analysis sottware that provides a variety of statistical analysis functions in an interactive manner.

In the practical application of RSM, it is necessary to develop an approximate model for the true response surface. The approximate model is based on observed data from the process or system and is an empirical model. The second order (quadratic) response surface model for response variable $y$ with $k$ regressors can be written as

$$
y=\beta_{0}+\sum_{i=1}^{k} \beta_{i} x_{i}+\sum_{i=1}^{k} \beta_{i n} x_{i}^{2}+\sum_{i=1}^{k-1} \sum_{i=2}^{k} \beta_{i j} x_{i} x_{i}+\varepsilon
$$

The above equation can be written in matrix notation as follows

$$
y=X \beta+\varepsilon
$$

where $\boldsymbol{y}:(n \times l)$ vector of observations, $X:\left(n \times n_{p}\right)$ matrix of the levels of the independent variables, $\beta:\left(n_{p} \times I\right)$ vector of the regression coefficients, $\varepsilon:(n \times l)$ vector of random error, $n$ : the number of observations, and $n_{p}$ : the number of terms in the model.

The purpose is to find the vector of least square estimators, $b$, that minimizes

$$
L=\sum_{i=1}^{n} \varepsilon_{i}^{2}=\varepsilon^{l} \varepsilon=(y-X \beta)^{r}(y-X \beta)
$$

which yields to the least squares estimator of $\beta$

$$
b=\left(X^{T} X\right)^{-1} X^{r} y
$$

The global fit and prediction accuracies of the response surfaces are assessed through statistical measures such as the t-statistic, or t-ratio, root mean square error (rms-error), variation (Myers and Montgomogery [37]). The t-statistic is determined by

$$
t=\frac{b}{\operatorname{se}\left(b_{1}\right)}
$$

where $b_{j}$ is the least squares estimators of $j^{\text {th }}$ regression coefficient and $s e\left(b_{j}\right)$ is the standard error of $b_{j}$ and it is given by

$$
\operatorname{se}\left(b_{j}\right)=\sigma_{a} \sqrt{C_{j j}}
$$


where $C_{J}$ is the diagonal element of $\left(X^{\tau} X\right)^{-1}$ corresponding to $b_{j}$. Here $\sigma_{a}$ is the adjusted rms-error (or rmserror predictor) incurred while mapping the surface over the data set. The quality of the tit of the different surfaces can be evaluated by comparing the adjusted rms-error value that is defined as:

$$
\sigma_{\mathrm{s}}=\sqrt{\frac{\sum \mathrm{e}_{\mathrm{i}}^{2}}{\mathrm{n}-\mathrm{n}_{\mathrm{i}}}}
$$

where $e_{i}$ is the error at $i^{\text {th }}$ point of the training data.

The accuracy of the models in representing the design space is gauged by comparing the values of the objective function at test design points, different from those used to generate the fit, with the empirical solution. The prediction rms-error, $\sigma$, for the test set is given by:

$$
\sigma=\sqrt{\frac{\varepsilon_{i}^{2}}{m}}
$$

In this equation $\varepsilon_{i}$ is the error at the $i^{\text {th }}$ test point and $m$ is the number of test points.

The coefficient of multiple determination $R^{2}$ measures the proportion of the variation in the response around the mean that can be attributed to terms in the model rather than to random error and it is determined by

$$
R^{2}=\frac{S S_{K}}{S S_{v \mathrm{v}}}=1-\frac{S S_{k}}{S S_{w}}
$$

$S S_{t:}$ is the sum of squares of the residuals $\left(=\sum_{i=1}^{n}\left(y_{i}-\hat{y}_{1}\right)^{2}\right)$ where $\hat{y}$ is the predicted value by the fitted model. $S S_{R}$ is the sum of squares due to regression $\left(=\sum_{i=1}^{n}\left(\hat{y}_{1}-\bar{y}\right)^{2}\right)$ where $\bar{y}$ is the overall average of $y_{i}$. $S S_{v}$ is the total sum of squares about the mean given by

$$
S S_{w}=S S_{t}+S S_{z}=\sum_{i=}^{n}\left(y_{1}-\bar{y}\right)^{2}
$$

where $\bar{y}$ is overall average of $y_{1}$.

$$
\begin{aligned}
& R_{u}^{2} \text { is an } R^{2} \text { value adjusted to account for the degrees of freedom in the model and is given by } \\
& R_{u}^{2}=1-\frac{S S_{t:} /(n-p)}{S S_{v v} /(n-1)}=1-\left(\frac{n-1}{n-p}\right)\left(1-R^{2}\right)
\end{aligned}
$$


Since $R^{2}$ increases as terms are added to the model, the overall assessment of the model may be better judged from $R_{u}^{2}$.

The polynomial-based response surface techniques are effective in representing the global characteristics of the design space. It can filter the noise associated with design dara. Since, the solution for the set of coefficients that best fits the training data is a linear least square problem, it is trivial compared to the solution for the NN coefficients, which is often a non-linear least square problem. The linearity of the polynomial-based RSM also allows us to use statistical techniques known as design of experiments (DOE) to find efficient training sets. On the other hand, depending on the order of polynomial employed and the shape of the actual response surface, the RSM can introduce a substantial error in certain region of the design space. An optimization scheme requiring large amounts of data and a large evaluation time to generate meaningful results is hardly useful.

\section{$2.2 \quad$ NEURAL NETWORKS (NN)}

Neural networks are massively parallel computational systems comprised of simple nonlinear processing elements with adjustable interconnections. Neural networks simulate human functions such as learning from experience, generalizing from previous to new data, and abstracting essential characteristics irom inputs containing irrelevant data (Greenman [14]). The processing ability of the network is stored in the inter-unit connection strengths or weights obtained by a process of adaptation to, or learning from, a set of training patterns. Training of a network requires repeated cycling through the data, each time adjusting the values of the weights and biases to improve performance. Each pass through the training data is called an epoch and the $\mathrm{NN}$ learns through the overall change in weights accumulating over many epochs. Training continues until the error target is met or until the maximum number of neurons is reached. In Figure 2, a neuron model with multiple inputs and bias is shown.

Accordingly, the input is transmitted through a connection that multiplies it with the weight related to that connection. The bias is similar to a weight except that it has a constant input of 1 . The effect of the product weight and input and the bias are added at the summing junction to form the net input for the transfer (or activation) function. In Figure 3. a multi-layer network is shown. A layer of network includes the combination of weights, the multiplication and summing operations. the biases and the transfer 
functions. In a layered neural network, neurons in every layer are associated with neurons in the previous layer in such a way that the outputs of an intermediate layer are the inputs to the following layer. The layer that produces the network output is called an output layer. All other layers are known as hidden layers.

Even though research on neural network started in early 1940s. NN became quite popular around 1980s with the introduction of multi-layered NN (Rumelhart et al. [56]) in a wide range of disciplines, including engineering. Over the last decade, NN approach has been used in the aerospace related industry. Illi et al. [21] examined the application of $\mathrm{NN}$ technology to an automated diagnostic and prognostic system for turbine engine maintenance. Preliminary results indicated that using NN to maintain diagnostics saves time and improves performance. Kangas et al. [24] used Back-Propagation NNs (BPNN) to monitor turbine engine performance and diagnose failures in real-time. The application of $\mathrm{NN}$ technology appears to hold great promise for enhancing the effectiveness of army maintenance practices. Huang et al.[20] developed and evaluated a multi-point inverse airfoil design method using NNs. It is shown that neural network predictions are acceptable for lift and moment coefficient predictions. Time dependent models that predict unsteady boundary layer development, separation, dynamic stall and reattachment are developed by Faller and Schereck [11] using VNs. It is demonstrated that NNs can be used to both predict and control unsteady aerodynamics effectively. Fan et al. [12] introduced a new approach for active laminar flow control that incorporates BPN. into a smart wall interactive flow control system. Convergence of the BPNN is investigated with respect to the complexily of the required function approximation, the size of the network in relation to the size of optimal solution and the degree of noise in the training data by Lawrence et al. [29]. The techniques and principles for the implementation of neural network simulators are also presented by Lawrence et al.[30]. Methods for ensuring the correctness of results avoiding duplication, automating common tasks, using assertions liberally, implementing reverse algorithms, employing multiple algorithms for the same task, and using extensive visualization are discussed. Efficiency concerns, including using appropriate granularity object-oriented programming, and pre-computing information whenever possible, are also studied. Norgaard et al. [40] used BPNN tor more effective aerodynamic designs during wind tunnel testing. Four different NNs are trained to predict coefficients of lift, drag, moment of inertia, and lift drag ratio $\left(C_{L}, C_{D}, C_{V}\right.$ and $\left.L D\right)$ from angle of attack and flap settings. Hybrid neural network optimization method is successtully applied to produce fast and reliable predictions of 
aerodynamic coefficients and to find optimal flap settings, and flap schedules. Ross et al. [55] applied BPNN to minimize the amount of data required to completely define the aerodynamic performance of a wind tunnel model. It is shown that the trained $\mathrm{V}$ has a predictive accuracy equal to or better than the accuracy of the experimental measurements using only $50 \%$ of the data acquired during the wind tunnel test. BPNN is employed for rapid and efficient dynamics and control analysis of flexible systems by Sparks and Maghami [60]. It is demonstrated that $\mathrm{NN}$ can give very good approximations to nonlinear dynamic components, and by their judicious use in simulations, allow the analyst the potential to speed up the analysis process considerably once properly trained. The high-lift performance of a multi-element airfoil is optimized by using neural-net predictions by Greenman [14].

BPNN have been successfully integrated with a gradient-based optimizer to minimize the amount of data required to completely define the design space of a three-element airfoil. It is shown that using NN reduced the amount of computational time and resources needed in high-lift rigging optimization. Greenman and Roth [15] also applied BPNN for high-lift performance of a multi-element airfoil and it is demonstrated that the trained NN predicted the aerodynamic coefficients within an acceptable accuracy defined to be the experimental error. Stepniewski and Jorgenson [6!] used a Singular Value Decomposition based node elimination technique and enhanced implementation of the Optimal Brain Surgeon algorithm to choose a proper $\mathrm{NN}$ architecture. It is demonstrated that combining both methods creates a powerful pruning scheme that can be used for tuning feed-forward connectionist models. Maghami and Sparks [34] \& [35] also demonstrated that the methodology they developed based on statistical sampling theory guarantees that the trained networks provide a designer-specified degree of accuracy in mapping the functional relationship. BPNN is used to fill in a design space of computational data in order to optimize flap position for maximum lift for a multi element airfoil by Greenman and Roth [16]. A Genetic Algorithm (GA) and gradient-based optimizer are used together with NN and it is found that the demonstrated method has a higher fidelity and a reduction in CPU time when compared to an optimization procedure that excludes GA. Approximation abilities of BPNN is addressed by Lavretsky [28]. A novel matrix method for multi-input-multi-output $\mathrm{VN}$ is introduced and it is shown that by allowing inner layer connections as well as connections between any layers, ordered $N \mathrm{~N}$ has superior interpolation ability when compared to conventional feed-forward VN. Stepniewski et al. [62] presented a new hybrid method that 
combines a bootstrap technique and a collection of stochastic optimization method such as GA for designing a NN. The method minimizes generalization error. It is demonstrated that the solutions produced by this method improve the generalization ability on the average of five to six times when compared to pruned methods.

All of the above listed references preferred to use BPNN among the other NN choices (Dernuth and Beale[8], Kosko[25], and Jang et al. [22]). This is due to the fact that BPNN attempts to have small number of neurons when compared to the other NNs. However, since BPNN is usually slower because at each step the error is propagated back to all the weights in the system, other NNs could be more efticient than BPNN for specific problems. This article reviews the works focusing on Radial-Basis NN (RBNN) and BPNN models developed by using Matlab (Dernuth and Beale [8]). A comparative study for radialbasis and back-propagation approaches is also included. Brief summaries of the two approaches are given in the following sections

\subsubsection{Back-propagation Networks (BPNN)}

Back-propagation networks are created by generalizing the Widrow-Hoff learning rule (Dernuth and Beale [8] and Kosko[25]) to multiple-laver networks and nonlinear differentiable transter functions. These networks are multi-layer networks with hidden layers of sigmoid transfer function and a linear output layer. The transfer tunction in the hidden layers should be differentiable and thus, either log-sigmoid or tansigmoid functions are commonly used. In this article, a single hidden layer with a tan-sigmoid transfer function, tansig (Figure 4), given as $\tanh (n)$, if $n$ is the input is considered. The maximum and minimum outputs of the function are 1 and -1 , respectively

The output of the function is given by

$$
a=\operatorname{tansig}(w \times X+b)
$$

where tansig is the transfer function, $\boldsymbol{w}$ is the weight vector, $\boldsymbol{X}$ is the input and $b$ is the bias. For BPNN, the initial weights and biases are randomly generated and then the optimum weights and biases are evaluated through an iterative process. The weights and biases are updated by changing them in the direction of down slope with respect to the sum-squared error of the network, which is to be minimized. The sum-squared error is the sum of the squared error between the network prediction and the actual values of the output. In 
BPNN (Figure $4 a$ ) the weights, $w_{l}$, and biases, $b_{i}$, in the hidden tansig layer are not fixed as in the case of RBNN. Hence, the weights have a nonlinear relationship in the expression between the inputs and the outputs. This results in a nonlinear regression problem, which takes a longer time to solve than RBNN Depending upon the initial weights and biases, the convergence to an optimal network design may or may not be achieved. Due to the randomness of the initial guesses, if one desires to mimic the process exactly for some purpose, it is impossible to re-train the network with the same accuracy or convergence unless the process is reinitiated exactly as before. The initial guess of the weights is a random process in Matlab. Hence to re-train the network the initial guess has to be recorded.

The number of neurons in the hidden layer of a back-propagation network is a design parameter. It should be large enough to allow the network to map the functional relationship, but not too large to cause overfitting. As a rule of thumb to choose the number of neurons in the hidden layer, Greenman [14] used $2 s+1$ where $s$ is the summation of total number of inputs and total number of outputs and Carpenter and Barthelemy [4] used $\mathrm{m}+\mathrm{l}$ where $\mathrm{m}$ is the number of nodes in the output layer. Once the number of neurons in the hidden layer is decided, the network design is reduced to adjusting the weighting coefficient matrices and the weighting bias vectors. These parameters tor BPNN are usually adjusted using a gradient method such as the Levenberg-Marquardt technique (Greenman [14], Norgaard et al. [40], Ross et al. [55], Sparks and Maghami [60]. Stepniewski et al. [62]). In Matlab, BPNV can be trained by using three different training functions, trainbp, trainbpx and trainlm. The first two are based on the steepest descent method. Simple back-propagation with trainbp is usually slow since it requires small learning rates for stable learning. Trainbpx, applying momentum or adaptive learning rate, can be considerably faster method than trainbp but trainlm, applying Levenberg-Marquardt optimization, is the most efficient since it includes improvement techniques to increase the speed and reliability of simple back-propagation networks. The Levenberg-Marquardt update rule is

$$
\Delta W=\left(J^{i} J+\mu I\right)^{-1} J^{r} e^{2}
$$

where $\Delta W$ is the change in weight, $J$ is the Jacobian matrix of the derivatives of each error with respect to each weight, i.e., $\frac{\partial e}{\partial w_{1}}, l$ is the identity matrix, $\mu$ is a scalar and $e$ is the error vector. If the scalar $\mu$ is large, the above expression approximates the steepest descent. while if it is small then the method reduces 
to the Gauss-Newton method. The Gauss-Newton method is taster and more accurate near an error minimum, so the aim is to shift towards the Gauss-Newton method as quickly as possible. Therefore, $\mu$ is decreased after each successful step and increased only when a step increases the error. The design parameters for trainlm are the number of neurons in the hidden layer, $S_{l}$, a user defined sum square error goal, and the maximum number of epochs. The training continues until either the error goal is reached, the minimum error gradient occurs, the maximum value of $\mu$ occurs, or the maximum number of epochs has been met.

\subsubsection{Radial-Basis Neural Networks (RBNN)}

Radial-basis neural networks are two-layer networks with a hidden layer of radial-basis transfer function and a linear output layer. The main advantage of this approach is the ability of keeping the mathematics simple and computational costs low due to linear nature of RBNN (Orr [41]). Outline of supervised learning, main application area for RBNNs and the least squares method used together with supervised learning with linear models are explained in detail in Orr [41]. Optimum of the regularization parameter of RBNN is also searched in this paper. A computational method for re-estimating the regularization parameter of RBNN, based on generalized cross-validation, is explained by Orr [42]. RBNN is designed in such a way that it can adapt the width of the basis function, and it is found that it can predict better than a similar RBNN with the fixed width basis function. Orr [43] explains improvements made for to forward selection and ridge regression methods. A methodology that is a cross between regression trees and RBNN is described. The size of RBNN is also optimized based on regularization parameter in Orr [42].

The transfer function for radial-basis neuron is radbas, which is shown in Figure 5. Radbas, given

as $e^{-n^{2}}$, where $n$ is the input, has maximum and minimum outputs of 1 and 0 respectively. The output of the function is given by

$$
a=\operatorname{radbas}(\operatorname{dist}(w, X) \times b)
$$

where radbus is the transter function, dist is the vector distance between the network's weight matrix, $w$, and the input vector, $X$ and $b$ is the bias. Radial-basis transter function radbas calculates its output according to $a=e^{-n^{2}}$ 
In a radial basis network (Figure 5a) each neuron in the radbas hidden layer is assigned weights, $w_{1}$ which are equal to the values of one of the training input design points. Therefore, each neuron acts as a detector for a different input. The bias for each neuron in that layer, $b_{t}$ is set to $0.8326 / s c$, where $s c$ is the spread constant, a value defined by the user. This defines the region of influence by each neuron. The training process is then reduced to the evaluation of the weights, $\boldsymbol{w}_{2}$, and biases, $b_{2}$, in the output linear layer, which is a linear regression problem. If the input to a neuron is identical to the weight vector, the output of that neuron is 1 , since the effective input to the transfer function is zero. When a value of 0.8326 is passed through the transfer function the output is 0.5 . For a vector distance equal to or less than $0.8326 / b$, the output is 0.5 or more. The spread constant defines the radius of the design space over which a neuron has a response of 0.5 or more. Small values of $s c$ can result in poor response in a domain not closely located to neuron positions, that is, for inputs that are far from the training data as compared to the defined radius, the response from the neurons will be negligible. Large values will result in low sensitivity of neurons. Since the radius of sensitivity is large, neurons whose weights are different from the input values by a large amount will still have high output thereby resulting in a flat network. The best value of the spread constant for some test data can be found by comparing $\sigma$ for networks with different spread constants.

In Matlab, radial-basis networks can be designed using two different design procedures, solverbe and solverb. Both procedures require a spread constant. sc, as a design parameter; i.e., the radius of the basis in the input space to which each neuron responds. Solverbe designs a network with zero error on the training vectors by creating as many radial-basis neurons as there are input vectors. Therefore, solverbe may result in a larger network than required and may fit the numerical noise. A more efficient design in terms of network size is obtained from solverb, which creates one neuron at a time to minimize the number of neurons required. At each epoch, neurons are added to the network until it satisties a user specified error goal. The design parameters for solverb are the spread constant, error goal, and the maximum number of epochs whereas it is only the spread constant for solverbe.

Radial-basis networks may require more neurons than a comparable BPNN. However, RBNN can be designed in a fraction of the time it takes to train the standard BPNN due to non-linear regression process of back-propagation networks. Theretore, RBVN are more efficient to train when there is a large 
amount of training data available. In Papila et al. [49], an effort is made to compare the accuracy and computing requirements between the radial-basis and back-propagation approaches with different sizes of training data. Vaidyanathan et al. [71] also investigated relative performances of RBNN and BPNN for gasgas injector and supersonic turbine. As will be discussed in the following sections, it is illustrated that among all the $\mathrm{NN}$ configurations, RBNN designed with solverb seems to be more consistent in performance for different data sets and RBNN, even when designed efficiently with solverb, tend to have many more neurons than a comparable BPNN with tan-sigmoid or log-sigmoid neurons in the hidden layer. The basic reason for this is the fact that the sigmoid neurons can have outputs over a large region of the input space, while radial-basis neurons only respond to relatively small regions of the input space. Configuring a RBNN often takes less time than that required for a BPNN because the training process of RBNN is a linear in nature.

\subsection{DESIGN OF EXPERIMENTS (DOE)}

In RSM, selecting the representation of the design space is a critical step because it dictates the distribution of the information available for constructing the response surface. It is well established that the predictive capability of RSM is greatly influenced by the distribution of sampling points in design space (Unal et al. [69] \& [70]). In order to select design points for training that minimizes the effect of noise on the fitted polynomial, design of experiment $(D O E)$ techniques can be applied. There are different types of design of experiments techniques in the literature as reported by Haftka et al. [19]. For example, Unal et al. [70] discussed the D-optimal design for the representation of the design space for a wing-body configuration of a launch vehicle. They showed that $D$-optimal design provides an efficient approach for approximating model building and multidisciplinary optimization. Papila and Haftka [48] also applied face centered composite design ( $F C C D$ ) to select the experiment points in the design space when approximating wing structural weight. Unal et al. [68] \& [69] studied response surtace modeling using orthogonal arrays $(O A)$ in computer experiments for reusable launch vehicles and illustrated that using this technique can minimize design, development, test and evaluation cost. Unal and Dean [67] studied the robust design method based on the Taguchi method (Unal and Dean [66] and Dean [7]) to determine the optimum configuration of design parameters for performance, quality and cost. They demonstrated that using such a 
robust design method for selection of design points is a systematic and efficient approach for determining the optimum configuration. Brief summaries of $F C C D, O A$, and D-Optimal designs are given below.

\subsubsection{Face Centered Cubic Design (FCCD)}

Face centered cubic design ( $F C C D$ ) creates a design space composed of eight corners of a cube, centers of the six faces and the center of the cube. Figure 6 shows FCCD selections for three design variables. The $F C C D$ yields $\left(2^{N}+2 N+1\right)$ points, where $N$ is the number of design variables. It is more effective when the number of design variables is modest, say, not more than 5 or 6 . The FCCD is used for fitting second-order response surface.

\subsubsection{Orthogonal Arrays (OA)}

An orthogonal array $(O A)$ is a fractional factorial matrix that assures a balanced comparison of levels of any factor or interaction of factors. Consider $\boldsymbol{A}$, a matrix with elements of $a_{1}^{l}$ where $j$ shows the row $\left(j=1,2 \ldots n_{r}\right)$ and $i$ shows the column $(i=1,2 \ldots n c)$ that $a_{1}^{\prime}$ belongs to, supposing that each $a_{1}^{\prime} \in Q=$ $\{0,1 \ldots q-1\} . A$ is called an orthogonal array of strength $t \leq n_{i}$ if in each $n_{r}$-row-by-t-column sub-matrix of

all $q^{\prime}$ possible distinct rows occur $\lambda$ times (Owen [4+]). Such an array is denoted by $O A\left(n_{r}, n_{r}, q, t\right)$ by Owen $[4]$.

Since the points are not necessarily at the vertices, the $O A$ can be more robust than the $F C C D$ in interior design space and are less likely to fail the analysis tool. Based on the design of experiment theory, orthogonal arrays can significantly reduce the number of experimental configurations.

\subsubsection{D-Optimal Design}

A D-Optimal design minimizes the generalized variance of the estimates, which is equivalent to maximizing the determinant of the moment matrix, $\boldsymbol{M}$ (Myers and Montgomery [37]).

$$
|M|=\frac{\left|X^{\tau} X\right|}{n^{n}}
$$


where $n$ is the number of observations and $n_{p}$ is the number of terms in the model.

The D-Optimal Design approach makes use of the knowledge of the properties of polynomial model in selecting the design points. This criterion tends to emphasize the parameters with the highest sensitivity (Haftka et al. [19]).

\subsection{OPTIMIZATION PROCESS}

\subsubsection{Search Procedure}

The entire optimization process can be divided into two parts: (1) RS/NN phase for establishing an approximation, and (2) Optimizer phase.

In the first phase, polynomials or NN models are generated with the available training data set. In the second phase the optimizer uses the RS/NN during the search for the optimum until the final converged solution is obtained. The initial set of design variables is randomly selected from within the design space. The flowchart of the process is shown in Figure 7.

The optimization problem at hand can be formulated as $\min \{f(x)\}$ subject to $l b \leq \mathrm{x} \leq u b$, where $l b$ is the lower boundary vector and $u b$ is the upper boundary vector of the design variables vector $x$. If the goal is to maximize the objective function then $f(x)$ can be written as $-g(x)$, where $g(x)$ is the objective function. Additional linear or nonlinear constraints can be incorporated if required. The optimization toolbox in Matab is used here employs a sequential quadratic-programming algorithm.

\subsubsection{Objective Function}

When attempting to optimize two or more different objective functions, conflicts between them arise because of the different relationships they have with the independent parameters. An equation expressing the relationship between opposing effects of pertormance and weight can be employed as a criterion to guide the optimization task. Both $\therefore N$ and polynomial-based RS techniques can handle such multi-criteria optimization tasks in a straightforward manner by building a composite response surface from individual response surtaces. Such a task would have been impossible without response surface. This composite response surface is referred to as the desirability function. The maximization of the composite 
function effectively provides a compromise between the individual functions. An average of some form is normally used to represent the composite function. A geometric mean is a solution, which gives a composite function of the form:

$$
D=\left(\prod_{i=1}^{i} d_{i}\right)^{1 / 1}
$$

where $D$ is the composite objective function, $d_{1}$ 's are normalized values of the objective functions and $l$ is the number of objective functions. Each of the $d_{i}$ are weighted depending upon the importance of the specific objective function. Figure 8 shows a typical trend for a desirability function with respect to the weighting factors.

Another way of constructing a composite function is to use a weighted sum of the objective functions. The composite function can then be expressed as:

$$
D=\sum_{i=1}^{1} \alpha_{i} f_{i}
$$

where $D$ is the composite objective function and $f_{i}$ 's are the non-normalized objective functions. The $\alpha_{1}$ 's are dimensional parameters that control the importance of each objective function.

\section{DESCRIPTION OF THE CASE STLDIES}

\subsection{GAS-GAS INJECTOR ELEMENT FOR ROCKET PROPLLSION}

Development of an optimization scheme for injector design called method $i$ (Methodology for Optimizing the Design of Injectors) has been reported by Tucker et al. [64] \& [65] Method $i$ is used to generate appropriate injector design data and then guide the designer toward an optimum design subject to his specified constraints. As reported, method $i$ uses the polynomial based RSM to facilitate the optimization. The RSM approach is to conduct a series of well-chosen experiments (empirical, numerical, physical or some combination of the three) and use the resulting information to construct a global approximation (response surface) of the measured quantity (response) over the design space. A standard constrained optimization algorithm is then used to interrogate the response surface for an optimum design. Neural network was also used in the design of shear co-axial injector element by Shyy et al. [57], and Tucker et al [64] \& [65] along with the polynomial-based RSM.. Three different injector types are 
considered, namely, shear co-axial injector element, impinging injector element, and swirl co-axial injector element.

\subsubsection{Shear Co-axial Injector Element}

The initial demonstration of method $i$ by Tucker et al. [64] focused on a simple optimization of a shear co-axial injector element (Figure 9) with gaseous oxygen $\left(G O_{2}\right)$ and gaseous hydrogen $\left(G H_{2}\right)$ propellants. The goal is to maximize the energy release efficiency, ERE while minimizing the chamber wall heat flux, $Q$. This is achieved by maximizing a composite objective function given by:

$$
D=\left(d_{t: R E} d_{Q}\right)^{1 / 2}
$$

where the normalized functions are defined as in Eqns (19) and (20). In the case where a response should be maximized, such as $E R E$, the normalized function takes the form:

$$
d_{E R E}=\left(\frac{E R E-A}{B-A}\right)^{\prime} \text { for } A \leq E R E \leq B
$$

where $B$ is the target value and $A$ is the lowest acceptable value. $d_{E R E}$ is set to $l$ for any $E R E>B$ and $d_{E R F}=$ $O$ for $E R E<A$. The choice of $s$ is made based on the subjective importance of this objective in the composite desirability function. In the case where a response is to be minimized, such as $Q$, the normalized function takes on the form:

$$
d_{Q}=\left(\frac{E-Q}{E-C}\right)^{\prime} \text { for } C \leq Q \leq E
$$

where $C$ is the target value and $E$ is the highest acceptable value. $d_{12}$ is set to $l$ for any $Q<C$ and $d_{t 2}=0$ for $Q>E . A, B, C$, and $E$ are chosen according to the designer's priorities or, as in the present article, simply as the boundary values of the domain of $E R E$ and $Q$. The value of $t$ is again chosen to reflect the importance of the objectives in the design. In the study carried out, $A$ and $B$ are equal to 95.0 and 99.9 , respectively. Values of $C$ and $E$ are equal to 0.48 and 1.1 , respectively.

The design data was generated using an empirical design methodology developed by Calhoon et al. [3]. These researchers conducted a large number of cold-flow and hot-fire tests over a range of propellant mixture ratios, propellant velocity ratios and chamber pressure for shear co-axial, swirl co-axial. 
impinging, and premixed elements. The data were correlated directly with injector/chamber design parameters, which are recognized from both theoretical and empirical standpoints as the controlling variables. For the shear co-axial element, performance, as measured by energy release efficiency, ERE, is obtained using correlations taking into account combustor length, $L_{c m m b}$ (length from injector to throat) and the propellant velocity ratio, $V N_{0}$. The nominal chamber wall heat flux at a point just dounstream of the injector, $Q_{n o m}$, is calculated using a modified Bartz equation and is correlated with propellant mixture ratio, $O / F$, and propellant velocity ratio, $V_{f} V_{o}$ to yield the actual chamber wall heat flux, $Q$. The objective in the initial demonstration of method $i$ was to maximize injector performance while minimizing chamber wall heat flux (lower heat fluxes reduce cooling requirements and increase chamber life) and chamber length (shorter chambers lower engine weight). The data used to generate the polynomials and train the network is given in Table A3-A5. The quality of the response surface and neural networks are decided using 20 additional design points different from those used to generate the models (Table A6).

\subsubsection{Impinging Injector Element}

The empirical design methodology of Calhoon et al.[3] uses the oxidizer pressure drop, $\Delta P_{o}$, fuel pressure drop, $\Delta P_{f}$, combustor length, $L_{\text {iomi }}$, and the impingement half-angle, $\alpha$ as independent variables. For this injector design, the pressure drop range is set to $10-20 \%$ of the chamber pressure due to stability considerations. The combustor length, defined as the distance from the injector to the end of the barrel portion of the chamber, ranges from $2-8$ inches. The impingement half angle is allowed to vary trom $15-$ $50^{\circ}$. Dependent variables include $E R E$ (a measure of element performance), wall heat flux, $Q_{m}$, injector heat flux, $Q_{i n j}$, relative combustor weight, $W_{\text {rel, }}$ and relative injector cost, $C_{\text {rel }}$.

The conditions selected tor this example are:

$$
\begin{aligned}
& P_{i}=1000 \mathrm{psi} \\
& M R=6 \\
& m_{i, i_{2}}=0.25 \mathrm{lb} / \mathrm{sec} \\
& m_{i t:}=0.042 \mathrm{lb} \mathrm{sec}
\end{aligned}
$$

The gaseous propellants are injected at a temperature of $540 \mathrm{R}$. As noted above. the empirical design methodology used to characterize the $E R E$ and $Q_{w}$ was developed by Calhoon et al. [3]. This 
methodology uses a quantity called the normalized injection momentum ratio to correlate the mixing at the different design points for the triplet element. They define this quantity as

$$
M R_{n}=\frac{2.3 m_{0} u_{0}}{m_{t} u_{t} \sin \alpha}
$$

The maximum mixing, and thus maximum ERE, occurs at an $M R_{n l}$ of 2.0 . Since the propellant mass flow rates are fixed, only the propellant velocities and the impingement half-angle influence the normalized injection momentum ratio. The velocities are proportional to the square root of the respective pressure drops across the injector, $\Delta P_{o}$ and $\Delta P_{j}$. For the flow conditions and variable ranges considered in this problem, $M R_{n i}$ ranges from 3.2 to 17.8. Accordingly, lowering $\Delta P_{o}$, raising $\Delta P_{j,}$ increasing $\alpha$, or some combination of these actions will increase ERE. The wall heat flux is correlated with the propellant momentum ratio as defined by

$$
M R=\frac{m_{0} u_{0}}{m_{f} u_{f}}
$$

For the F-O-F triplet element, i.e. the impingement injector element, the maximum wall heat flux occurs at a momentum ratio of approximately 0.4 . High heat flux is the result of over-penetration of the fuel jet, which produces a high $O / F$ in the wall region. For the flow conditions and variable ranges considered in this effort, $M R$ ranges from 1.06 to 2.11 . Hence, increasing the value of this ratio by either increasing $\Delta P_{o}$ or decreasing $\Delta P_{i}$ lowers the wall heat flux.

The heat flux seen by the injector face. $Q_{n}$, is qualitatively modeled by the impingement height, $H_{\text {impinge. }}$ The notion being that, as the impingement height decreases, the combustion occurs closer to the injector face, causing a proportional increase in $Q_{i n^{\prime}}$. Thus, for the purposes of this exercise. $Q_{i n j}$ is modeled as the reciprocal of the $H_{\text {impinge. }}$ Impingement height is a function of $\alpha$ and $\Delta P_{f}$. Reference to Figure 10 shows that as $\alpha$ is increased, $H_{\text {imp:nge }}$ is shortened. The dependence of $H_{\text {inpinge }}$ on the fuel orifice diameter. $d_{f}$, and thus, $\Delta P_{f}$, results from making the freestream length of the fuel jet. $L_{j,}$, a function of $d_{f}$. For each $\Delta P_{l}, L_{i s}$ was set to six times $d_{\text {, }}$ for an impingement halt-angle of $30^{\circ}$. So. as $d_{i}$ increases (corresponding to

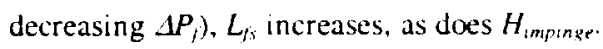

The models for $W_{r e l}$ and $C_{r e l}$ are simple but represent the correct trends. $W_{r e l}$ is a function only of $L_{c i m b}$, the combustor length from injector face to the end of the chamber barrel section. The dimensions of 
the rest of the thrust chamber assembly are assumed to be fixed. So, as $L_{c o m b}$ increases, $W_{\text {rel }}$ increases accordingly. The model for $C_{\text {rei }}$ is based on the notion that smaller orifices are more expensive to machine. Therefore, $C_{r e l}$ is a function of both propellant pressure drops. As the $\Delta P$ increases, the propellant velocity through the injector increases and the orifice area decreases. So, as either, or both, $\Delta P_{o}$ and $\Delta P_{f}$ increase, $C_{\text {rel }}$ increases.

The system variables given above and independent variables (constrained to the previously noted ranges) are used to generate the design data for element optimization studies. Since propellant momentum ratio is an important variable in the empirical design methodology, a matrix of momentum ratios was developed over the 100-200psi propellant pressure drop range. The matrix of 49 combinations of fuel and oxidizer pressure drops is shown in Table A7 where momentum ratios range from 1.06 to 2.11 . Nine pressure drop combinations, eight around the border and one in the middle, were selected for use in populating the design database. These nine points are highlighted in Table A 7 in bold type

Detailed design results for the case with both $\Delta P_{o}$ and $\Delta P_{f}$ at 200psi are shown in Table A8.

Similar data was generated for the other eight pressure drop combinations. There are 20 combinations of $L_{c u n t b}$ and $\alpha$ for each $\Delta P$ combination, making a total of 180 design points selected. Seventeen of these were outside the database embodied by the empirical design methodology, resulting in 163 design points actually being evaluated. The data trends are as expected. $E R E$, for a given $A P$ combination, increases with increasing $L_{\text {comb }}$ and $\alpha$. The increased $L_{c o m b}$ provides more residence time tor the propellants to mix and burn. Increasing $\alpha$ increases the radial component of the injected fuel, thus providing better mixing. The wall heat flux is constant for a given $\Delta P$ combination. Impingement height increases with increasing $\alpha$. Relative combustor cost increases with increasing $L_{\text {comb }}$ and the relative injector cost is constant for a given $\Delta P$ combination.

\subsubsection{Swirl Co-axial Injector Element}

The chamber pressure, mixture ratio, and propellant flow rates selected for this example are:

$$
\begin{aligned}
& P_{i}=1000 \mathrm{psi} \\
& M R=6 \\
& m_{i, j m_{m}}=0.25 l b_{m} / \mathrm{sec} \\
& m_{i ; m_{i}}=0042 l b_{m} \mathrm{sec}
\end{aligned}
$$


The gaseous propellants are injected at a temperature of $540 \mathrm{R}$. Reference to Figure 11 shows that the $\mathrm{GO}_{2}$, flowing in the center post of the element, exits the element with both radial and axial velocity components. This effect is achieved by introducing the $\mathrm{GO}_{2}$ tangentially into the center post through small slots. When the $\mathrm{GO}_{2}$, under hydrostatic head, is forced through the tangential slots, part of the pressure head is converted into a velocity head, causing a rotational velocity in the element. For a specified $\Delta P_{\text {, }}$ and swirl angle, $\Theta$, the number and size of tangential slots, the discharge coefficient, the $\mathrm{GO}_{2}$ center post diameter, $d_{o}$, and the radial and axial $G O_{2}$ velocity components, $V_{o r}$ and $V_{o a}$ are calculated. These quantities are then used to determine the dependent variables for each design condition.

The element ERE, calculated according to the empirical design methodology of Calhoon et al.[3], is a function of all four independent variables noted above. A cold flow mixing efficiency, $E_{m, 4 t}$, for $\Theta=90^{\circ}$, is correlated by:

$$
E_{m, 90}=100-5 \ln \left\lfloor\frac{K_{s}}{L_{\text {coid }} / d_{v}}\right\rfloor
$$

The cold flow mixing length, $L_{\text {toid }}$, is correlated from a known chamber length, $L_{c u m b}$. The $G \mathrm{O}_{2}$ post diameter, $d_{0,}$, is a function of $\Delta P_{1,}$ and $\Theta$. Smaller values of $d^{\prime}$, correspond to large values of $\Delta P^{\prime}$, and smaller swirl angles. The empirical swirl factor, $K_{j}$, is a function of the normalized differential injection velocity, $\left(V_{,}-V_{0}, V_{o} . K_{\text {i }}\right.$ increases with increasing normalized differential injection velocity for the range of propellant velocities considered in this effort. For fixed propellant mass flow rates, the velocities $V_{o}$ and $V_{i}$ are functions of their pressure drops across the injector, $\Delta P_{v}$ and $\Delta P_{f}$, respectively. For a given $\Delta P_{u}, V_{o}$ also depends on the swirl angle. Lower $V_{o}^{\prime}$ 's are a product of higher swirl angles. Cold flow mixing is thereby enhanced with higher values of $V_{0}$ (i.e. $\Delta P_{b}$ ) and $L_{r(m m b)}$. Lower values of $V_{i}$ (i.e. $\Delta P_{j}$ ) and $\Theta$ also tend to enhance cold flow mixing.

A tractional factor, $f_{s}$, is applied to $E_{m, 4}$ to account for the lower levels of cold flow mixing found with swirl angles less than $90^{\circ}$. The resultant measure of cold flow mixing, $E_{m}, \theta$ is a product of $E_{m,(w)}$ and $f_{s}$. This factor, for a given design, is a function of the normalized differential injection velocity and the ratio of radial to axial $\mathrm{GO}_{2}$ velocity, $V_{r,}, V_{\text {tru }}$. Increasing values of both quantities increase $f_{,}$, with a value of $f_{i}=1$ 
being found at $V_{o r}, V_{o u}=1\left(\Theta=90^{\circ}\right)$ for all values of $\left(V_{f}-V_{o}\right) / V_{o s}$. Larger values of $f_{s}$ increase cold flow mixing. These values are found at low $\Delta P_{D}$ and high $\Delta P_{f}$ and $\Theta$. There is no dependency of $f_{;}$on chamber length. These trends are opposite those noted above. Finally, ERE is proportional to $E_{m}, \theta$

The wall heat flux $Q_{w}$, is correlated with the propellant momentum ratio as defined by:

$$
M R=\frac{m_{0} u_{0}}{m_{f} u_{f}}
$$

The wall heat flux curve from the Calhoon et al. [3] methodology is fairly flat, varying only about $10 \%$ from high to low for the range of pressure drops considered in this effort. $Q_{w}$ decreases with increasing $V_{o}$ (high $\Delta P_{o}$ and low $\Theta$ ) and decreasing $V_{f}$ (low $\Delta P_{f}$ ). That $Q_{w}$ would decrease with increasing $V_{o}$ is counter to intuition. It seems that high values of $V_{o}$, for any $\Theta$, would result in higher mixture ratios in the wall region, as is the case for liquid $\mathrm{O}_{2}$. Calhoon et al. [3] do not discuss this effect.

The heat flux seen by the injector, $Q_{i n}$, is actually modeled by the distance from the injector at which the propellant streams intersect. This axial distance is measured at the radial position corresponding to the center of the co-axial fuel annulus, or gap. It is here that the streams begin to mix and burn. This measure is qualitative, but captures the trend that higher injector heat fluxes occur the nearer the injector that the combustion begins. The axial distance is affected directly by the swirl angle, and indirectly by the propellant pressure drops. $Q_{i n j}$ decreases with decreasing swirl angle, increasing $\mathrm{GO}_{2}$ pressure drop and decreasing $\mathrm{GH}_{2}$ pressure drop. Swirl angle has the largest effect, while $\Delta P_{\text {o }}$ is the least significant factor.

The relative combustor weight, $W_{r e l}$, is simply a function of the combustor length, $L_{c o m b}$, the distance from the injector to the end of the barrel portion of the chamber. The longer the combustor, the more it weighs.

The relative injector cost, $C_{r e l}$, is a function of the fuel gap width and the width of the tangential slots used to induce the swirl in the $\mathrm{GO}_{2}$ center post. Larger values of both variables result in lower machining costs, and thus lead to lower injector cost. The fuel gap width increases with increasing $\Delta P_{n}$, and decreasing values of $\Delta P_{. /}$and $\Theta$. Swirl slot width increases with lower values of $\Delta P_{o}$ and $\Theta$. Overall, $C_{\text {ret }}$ decreases with increasing $\Delta P_{o}$ and decreasing $\Delta P_{f}$ and $\Theta$. Fuel pressure drop and swirl angle are the most significant factors. 
A matrix of propellant pressure drop combinations was developed and nine combinations were selected for use in populating the design database. There are 20 combinations of $L_{c m m b}$ and $\Theta$ for each $\Delta P$ combination, making a total of 180 design points selected.

In the work by Tucker et al. [64] \& [65], method $i$ uses the Response Surface Method (RSM) to find optimal values of $E R E, Q_{m}, Q_{i n j}, W_{r e l}$ and $C_{r e l}$ for acceptable values of $\Delta P_{m}, \Delta P_{j}, L_{t r m b}$ and $\Theta$. The approach of RSM is to perform a series of experiments, or numerical analyses, for a prescribed set of design points, and to construct a response surface of the measured quantity over the design space. In the present context, the five responses of interest are $E R E, Q_{w}, Q_{i n j}, W_{r e t}$ and $C_{r e l}$. The design space for each element consists of the set of relevant design variables $\Delta P_{o}, \Delta P_{f}, L_{c u m b}$ and $\Theta$. The response surfaces are fit by standard least-squares regression with a quadratic polynomial using JMP [23]. A backward elimination procedure based on t-statistics is used to discard terms and improve the prediction accuracy. Five full quadratic response surfaces are constructed by using JMP.

In the current case, it is desirable to maximize ERE and while simultaneously minimizing $Q_{w}, Q_{n_{j}}$, $W_{\text {rei }}$ and $C_{\text {rel }}$.

\subsection{SUPERSONIC TURBINE FOR REUSABLE LAUNCH VEHICLES}

Supersonic turbines that drive fuel or oxidizer turbopumps in rocket engines are of great interest to the next generation space propulsion industry, including the Reusable Launch Vehicles (RLV). They are complex, high-speed devices that produce shaft power by ducting the flow of hot gasses over specially shaped blades on a wheel. For rocket engine applications, maximizing the vehicle payload for a given turbine operating condition is the ultimate goal. The flow path should be designed in such a way that it wastes less energy so that turbine temperatures or the mass flow rate can be reduced, or the turbine can be made smaller, increasing the etficiency (or specific impulse) of the rocket engine. Any gain in turbine efficiency will be reflected in reduced propellant consumption, resulting in an increase in the payload. However, higher turbine performance usually entails multistage designs, which are heavier. The design of a supersonic turbine often involves a considerable number of design variables with structural and aerodynamic constraints. With the number of design parameters involved, the overall procedure of design optimization of supersonic turbines becomes a challenging task. 
Papila et al. [50] have conducted a global optimization investigation to perform the preliminary design of the supersonic turbines, including the selection of the number of stages and design variables. From 1- to 2- to 3-stage turbines, the number of design variables increases substantially. In shape design, from vane to blade, from stage to stage, and from 2-D to 3-D, not only does the number of design variables increase, but also the interactions among design variables become more complicated. Papila et al. [50] intended to investigate the individual, as well as collective effects of design variables by varying the design scope systematically. Vaidyanathan et al. [71] have used the data of the 1-stage turbine to conduct a comparative study between RSM and NN.

For preliminary design stage, single-, two- and three-stage turbines are considered. The design variables can be separated into two categories, one related to geometry and the other to performance. They are summarized as follows:

(I) Geometric inputs: The geometric inputs are needed to layout the turbine meridional geometry, e.g., mean diameter, last rotor annulus area, blade height ratio between the $1^{\text {st }}$ vane and the last rotor blade (linear distribution of blade heights is assumed between the $1^{\text {st }}$ vane and the last rotor blade), vane and blade axial chords

(2) Performance inputs: The performance inputs are needed to calculate the turbine efficiency, e.g., speed $(R P M)$, number of stages, blade row reaction, and work split (if more than I stage is investigated).

For single-stage turbine. 6 design parameters (Table 3) are selected. These are (1) the mean diameter, (2) speed ( $R P M)$, (3) exit blade annulus area, (4) vane axial chord, (5) blade axial chord, (6) stage reaction.

For 2-stage turbine, there are 11 design parameters (Table 3), namely, (1) the mean diameter, (2) $R P M,(3)$ exit blade annulus area, (4) Ist blade height (\% of exit blade), (5) $1^{\text {st }}$ vane axial chord, (6) $1^{\text {st }}$ blade axial chord. (7) $2^{\text {nd }}$ vane axial chord, (8) $2^{\text {nd }}$ blade axial chord, (9) $1^{\text {st }}$ stage reaction, (10) $2^{\text {nd }}$ stage reaction, and (11) $1^{\text {st }}$ stage work fraction. Note that $2^{\text {nd }}$ stage work traction is not a design parameter since it can be calculated by using $1^{\text {st }}$ stage work fractions, i.e., $w_{f 2}=1-w_{f l}$.

There are 15 (Table 3) design parameters for 3-stage turbine. These are (1) mean diameter, (2) speed $(R P M),(3)$ exit blade annulus area, (4) $1^{\text {st }}$ blade height (\% of exit blade), (5) $1^{\text {st }}$ vane axial chord, (6) $1^{\text {st }}$ blade axial chord, (7) $2^{\text {nd }}$ vane axial chord, (8) $2^{\text {nd }}$ blade axial chord, (9) $3^{\text {rd }}$ vane axial chord, (10) $3^{\text {rd }}$ 
blade axial chord, (11) $1^{\text {st }}$ stage reaction, (12) $2^{\text {nd }}$ stage reaction, (13) $3^{\text {rd }}$ stage reaction, (14) $1^{\text {st }}$ stage work fraction, (15) $2^{\text {nd }}$ stage work fraction. Note that $3^{\text {rd }}$ stage work fraction is not a design parameter since it can be calculated by using $1^{\text {st }}$ and $2^{\text {nd }}$ stage work fractions, i.e., $w_{f 3}=1-\left(w_{f 1}+w_{f 2}\right)$.

The composite objective function chosen by Papila et al. [50] for design optimization corresponds to the payload increment, $\Delta p a y$, versus turbopump efficiency and weight. The relation between $\Delta p a y$ and these two parameters can be developed as follows based on mission profile studies, engine balance perturbation and some detailed turbopump layout and stress information gained from other proprietary programs.

$$
\Delta \text { pay }=c_{1} \times\left(\eta-\eta_{b}\right) \times 100-\left(W-W_{b}\right)
$$

where $\eta_{b}$ is the baseline efficiency and $W_{b}$ is the baseline weight. The constant $c_{\text {, }}$ indicates that for every point in efficiency gained, the amount of payload capacity of the RLV is increased $c_{t}$ per turbopump Therefore, Apay function represents the amount of increase in payload capacity. The results of both payload increment based and composite desirability function based optimization are illustrated for 1, 2, and 3-stage designs. The results of both payload increment based- and composite desirability function-based optimization are illustrated for 1,2, and 3-stage designs in the following chapters.

Two structural constraints are considered by Papila et al. [50]. In axial turbines the product of the blade exit annulus area and the $R P M$ square, i.e., $A N^{2}$ is an indication of the blade centrifugal stress, which should bind the speed of the turbine. In addition, the disk stresses are also a restriction. In turbomachinery industry, the maximum stress value due to disk burst is often represented by a pitchline velocity limit, i.e.. $V_{\text {purh }}$. The pitchline velocity can be calculated by muttiplying $R P M$ and the mean radius.

\subsection{TURBULENT PLANAR DIFFUSER}

The goal was to accomplish maximum pressure recovery by optimizing the wall contours. The flow is incompressible and fully turbulent with a Reynolds number of $10^{5}$, based on the inlet throat halfwidth, $D$. The overall geometry is defined by the ratio of inlet and outlet areas, and the diffuser length to height ratio. In this study the length to height ratio is fixed at 3.0, and the area ratio at 2.0. The shape of the diffuser wall is designed for optimum performance, with tive design variables represented by B-splines The CFD model is based on the full Reynolds-averaged Navier-Stokes equations, with the $k \cdot \varepsilon$ two-equation 
turbulence model in closure form. At the inlet of the flow domain, a uniform flow distribution is specified. Detailed discussion of this study can be found in Madsen et al. [33].

\subsubsection{Objective}

The dimensionless pressure recovery coefficient $C_{p}$ is introduced as the objective function to be maximized

$$
F=C_{p}=\frac{\Delta p}{\frac{1}{2} \rho u_{i n !}^{2}}
$$

Here $\Delta p$ is the static pressure difference between channel cross sections up- and downstream of the diffuser respectively, $\rho$ is the fluid density, and $u_{i m}$ is the inlet mean velocity. Inlet and outlet static pressures are averaged. even though the pressure distribution is nearly uniform due to well-developed flow at the considered cross sections. The CFD model uses a symmetry condition along the channel center axis, and has a computational mesh consisting of $120 \times 50$ cells including a long outlet section to establish a fully developed exit profile. The overall geometry of the two-dimensional planar diffuser, see Figure 12 , is defined by the ratio of inlet and outlet areas, $A R$, and the diffuser length/height ratio, $L=D$, where $L$ is the axial length of the diffuser. In this study the $L=D$-ratio fixed at 3.0. and the area ratio $A R$ at 2.0. Expressed in terms of the inlet half-width $\mathrm{D}$, the horizontal position of the inlet is $1 D$, while the horizontal position of the outlet is $10 D$. The shape of the diffuser wall is designed for optimum performance, and to this end two separate cases of wall parameterizations are tried: (1) a two design variable case, where a polynomial describes wall shapes, and (2) a five design variable case that uses B-splines. Even though two different curve descriptions are used in the two cases, the most noteworthy difference seen from the point of view of the RSM lies in the problem size.

\subsubsection{Geometric Representation}

For shape parameterization in more variables, $B$-splines were preferred to natural splines (piecewise polynomials), although the latter technique is closer to the polynomial representation. B-splines excel in the predictable way that control points influence curve shape, and in the local control, which 
prevents small changes in a control point position from propagating over the entire curve. Combined with low computational cost, these advantages have contributed to $B$-spline curves becoming a standard geometric modeling technique in computer-aided design.

A $B$-spline is given in a parametric form as $p(u)$ :

$$
p(u)=\left[\begin{array}{c}
x(u) \\
y(u)
\end{array}\right]=\sum_{i=0}^{n} P N_{i, k}(u)
$$

A set of blending functions $N_{t, k}$ combines the influence of $n+l$ control points $\boldsymbol{P}_{l}$, over the range of the parametric variable $u$. The blending functions $N_{t, k}$ are recursively determined polynomials with degree $k-l$, where the parameter $k$ dictates the order of continuity of the curve, and thus how many control points influence a curve segment. In this work $k$ is 8 , which corresponds to $C^{6}$-continuity. The number of control points is 8 as well-two endpoints, five design variables and one point used for prescribing the inlet slope.

$B$-splines have an approximating nature, in that they do not necessarily pass through control points, except for fixed curve endpoints. The slope at a curve endpoint is tangential to a straight line connecting the endpoint and the first control point, and may be prescribed by placing an additional fixed control point near the endpoint.

Experimental and numerical evidence indicates that maximum pressure recovery in diffusers occurs at the border of appreciable flow separation. For this reason, strongly separated diffuser tlows should be avoided, which makes it reasonable to restrict the design space to monotonic wall shapes. While the approximation accuracy does of course benefit from the reasonable design space approach, it is equally important in the present example that monotonicity constraints eliminate convergence problems associated with CFD-analysis of odd. non-monotonic designs.

The parametric form in which $B$-splines are defined makes it non-trivial to derive monotonicity constraints analytically, so instead a constraint approximation $\hat{G}$ was set up in the form of a response surface for the minimum wall slope $G$. Then, observing the inequality constraint $G \geq 0$ implies a positive wall slope and thus monotonicity throughout. Since $B$-splines are inexpensive to generate, $9^{5}(59049) B$ splines were computed (this took just a few seconds) and used for fitting a quadratic response surface. The approximation to the monotonicity constraint precludes some designs that satisfy the exact monotonicity 
requirement. However, the effect of these inaccuracies on the solution of the optimum design problem is negligible.

The regression analysis, to find 21 polynomial coefficients in five dimensions, is based on a 35 point D-optimal design. The surplus of analyses is generally required for reducing the sensitivity to numerical noise and to errors due to the simplified representation as a quadratic polynomial. Again, a pool of candidate points was created, this time using nine levels for each variable, (values ranging from 0.0 to 1.0), and then checking the monotonicity of the $B$-splines for each of the $9^{5}=59049$ designs. Observe that limiting the $y$-coordinate of the control points to a variation in the range $[0: 0 ; 1: 0]$ is a somewhat artificial requirement, as monotonic shapes exist with coordinates slightly outside this range. A total of 20864 points are monotonic in wall shape. This relatively large percentage of acceptable cases reflects the smoother nature of approximating curves. Had a non-segmented polynomial curve representation been used, the condition of monotonicity in the control points would alone have reduced the number of feasible design points to less than $1 \%$ of those inside a five dimensional box. As in the two-design-variable case, the subset of D-optimal points was found using the $J M P$-software.

\subsection{LOW REYNOLDS NUMBER WING MODEL}

\subsubsection{Training Data}

The aerodynamic model, a rectangular wing with a NACA 5405 airtoil cross-section (Figure 13) is designed for low Reynolds Number $\left(R e=10^{t}-10^{6}\right)$ flows. Since airfoil performance decreases at low Reynolds Number flights, attempts to shrink the overall aircraft size while trying to keep sufficient lifting areas, result in low aspect ratio wing planforms. As aspect ratio decreases, the percentage of the wing area affected by the tip vortices increases, creating a 3-D flow field over most of the field. Therefore, the analysis of such flows should consider the effects on performance and the effects of both the airfoil geometry (such as maximum camber) and the wing geometry (such as aspect ratio). In this study, the aerodynamic analysis is based on a 3-D potential tlow solver, PMARC, and a 2-D coupled inviscid-viscous flow solver, $X F O l L$ : The lift coefficients, $C_{l}$, and drag coefficients, $C_{D}$, for various maximum camber, $y_{C}$. aspect ratios. $A R$, and angles-of-attack, $\alpha$, at fixed Reynolds number, $R e=2 \times 10^{5}$, and thickness ratio, $y_{i}=5 \%$, are used to correlate the aerodynamic performance, measured by the power index, $C_{L}^{3 / 2} / C_{D}$, which 
appears explicitly in steady flight required-power equation. Aspect ratio and maximum camber form the input vector, $p$ and $C_{t}^{3 / 2} / C_{b}$ forms the target output vector, $a$, as shown below.

$\mathrm{p}=\left[\begin{array}{l}\mathrm{AR} \\ y_{\mathrm{c}}\end{array}\right]_{2 \times \mathrm{R}} \mathrm{a}=\left[\mathrm{C}_{\mathrm{L}}^{3 / 2} / \mathrm{C}_{\mathrm{D}}\right]_{\times \mathrm{R}}$

where $R$ is the number of input vectors of the training data.

For the 3-D wing case, the maximum camber varies between 0.0 and 0.1 and the aspect ratio varies between one and five. Three different training data sets are used out of the available data as shown in Table A1. Table A2 summarizes the test data sets used for prediction for this case. A simulation, referred in these tables, consists of two input variables: $A R$ and $y_{c}$ and the output variable: $C_{L}{ }^{3 / 2} / C_{D}$.

\section{ASSESSMENT OF DATA PROCESSING AND OPTIMIZATION CAPABILITIES}

Of all the cases considered in this article, the impingement injector element, swirl co-axial injector element, 2-stage supersonic turbine and turbulent flow diffuser help understand the effectiveness of using polynomial based RSM. Shear co-axial injector element, 1-stage turbine and two-dimensional wing model are used to carry out a comparative study between RSM and NN. The size of the data set used varies trom modest to large (from 9 to 2235 data).

In the following, we synthesize the studies of Papila et al. [49] \& [50], Madsen et al.[33], Shyy et al. [57], Tucker et al. [64]\& [65] and Vaidyanathan et al.(71]. We first review the data processing capabilities then evaluate the performance of the optimization techniques. For both NN and polynomials, one needs to first decide most appropriate constructions for a given data set. For the NN, the choices are usually (1) the number of neurons, and (2) the error goals. Furthermore, the spread constant (for RBNN) and the number of hidden layers (for BPNN) can be specified. In this article, the BPNN and RBNN will be limited to the 2layer form. 


\subsection{SHEAR CO-AXIAL INJECTOR}

\subsubsection{Polynomial Fits}

According to the injector model developed by Calhoon et al. [3], injector performance, as measured by $E R E$ depends only on the velocity ratio, $V, N_{0}$, and combustion chamber length, $L_{c o m b}$. Examination of the original data set in Table A3-A6 indicates 15 distinct design points for ERE. Since chamber wall heat flux is dependent only on velocity ratio, $V / N_{o}$, and oxidizer to fuel ratio, $O / F$, there are 9 distinct design points for $Q$. The design space for this effort is depicted in Figure 14. For ERE, the 5 distinct chamber lengths offer the potential for a fourth-order polynomial fit in $L_{c o m b}$, while the three different velocity ratios limit the fit in $V N_{o}$ to second order. Quadratic and cubic response surfaces for both $E R E$ and $Q$ have been generated for evaluation. These response surfaces represent reduced models accomplished by term elimination from the full surface using t-statistics as described earlier. The above-noted limitations on the data cause the cubic surfaces to be third order in $L_{\text {cumb }}$ only.

Based on the adjusted RMS error, Vaidyanathan et al.[71] have concluded that the cubic fit is more accurate than the quadratic fit for ERE. The adjusted RMS error for the quadratic and cubic response surfaces of $E R E$ are 0.211 and 0.083 , respectively. The cubic fit, by this measure, is superior for $E R E$. However, the error is almost identical in the case of $Q$ for both the quadratic $(0.039)$ and cubic $(0.040)$ surfaces, perhaps due to the very small number of points available for the curve fit. The additional terms in the cubic fit relative to the quadratic fit do not improve the mapping of the response surface for $Q$.

\subsubsection{Construction of RBNN}

In the case of the injector design there are two objectives, namely $E R E$ and $Q$. Figure 15 gives the variation of $\sigma$ for the network designed with solverbe for these objective functions. In case of solverb the error goal during training also defines the accuracy of the network. An objective of fitting a numerical model is to remove the noise associated with the data. A model, which maps exactly as solverbe does, will not eliminate the noise, whereas solverb will. Figure 15 shows that for low values of spread constant, the $\mathrm{NN}$ has a poor performance. As the spread constant increases $\sigma$ asymptotically decreases. However, as demonstrated by Figure 15 a the performance of the network can deteriorate for higher values of the spread 
constant. The region with a large variation in $\sigma$ is highly unreliable because this indicates a high sensitivity of the model to a small variation of spread constant and possibly the test data, in this region. Hence the desirable spread constant is selected from the region where the performance of the network is relatively consistent.

Figure 16 gives the variation of $\sigma$ for the network design with solverb for the objective functions of $E R E$ and $Q$. It also shows the influence of error goal on the network. Generally if a network maps the training data accurately it can be expected to perform efficiently with the test data. However, accurately mapping noisy data may result in poor prediction capabilities for the network. The variation in the performance is not significant except for the $E R E$ and $Q$ network (Figure 16), where the poor performance of the network at high values of spread constant improves for a larger error goal. This may indicate the presence of noise in the data for $E R E$, which solverb is able to eliminate with an appropriate error goal. Figure 17 shows variations in number of epochs and $\sigma$ with the variation of error goal for a given spread constant when RBNN is designed with solverb. The number of neurons in the network is one more than the number of epochs. One expects that as the error goal increases the number of epochs becomes smaller and the network performs less accurately as in Figure $17 \mathrm{a}$ and $\mathrm{b}$.

When choosing an appropriate network the above-mentioned features have to be considered. The performance of the constructed $N N$ is best judged by comparing the prediction error as given in Eqn. (8) for different networks. Using solverbe. networks are designed with varying spread constants and the one that yields the smallest error is selected. When solverb is used, networks are designed for different spread constants and error goals. The network that gives the smallest error for the test data is used. The details of the networks selected are discussed in later sections.

\subsubsection{Evaluation of Polynomial and NN for Data Processing}

The polynomial and NN-based RSM are constructed using the training data. The test data is then employed to select the best polynomial or NN. Specifically in polynomial-based RSM, the difference between the polynomial and the training data, as given by $\sigma_{a}$, is normally used to judge the performance of the fit. The additional use of the test data helps to evaluate the performance of different polynomials over 
design points not used during the training phase. This gives a complementary insight into the quality of the polynomial model over the design space. For both the rocket engine components, different polynomials were tried. Table 4 and 5 compare the performance of different polynomials used to represent the two objective functions of the injector case, $E R E$ and $Q$. Starting with all the possible cubic terms in the model, revised models are generated by removing and adding terms. Similar kind of analysis is also done for the turbine case. The best polynomial is selected based on a combined evaluation between $\sigma_{a}$ and $\sigma$.

For the NN, the test data helps evaluate the accuracy of networks with varying neurons in BPNN and varying spread constant in RBNN. Thus the test data are part of the evaluation process to help select the final NN. Based on the RSM or NN model, a search for optimum design is carried out using a standard, gradient-based optimization algorithm over the response surfaces represented by the polynomials and trained neural networks.

A reduced quadratic and an incomplete cubic response surfaces are used for the two objective functions. The first model in Table 4 and the sixth model in Table 5 are the selected cubic models for $E R E$ and $Q$, respectively. There is no noticeable improvement amongst the remaining cubic models for $E R E$. For $Q$, the selected model is the best in terms of $\sigma_{\mathrm{a}}$ although there are other models with identical values of $\sigma$.

The radial basis networks designed with solverbe are the largest with 15 neurons in the hidden layer for $E R E$ network and nine neurons for the $Q$ network. Solverb designs a network for $E R E$ with 14 neurons in the hidden layer and a network for $Q$ with eight neurons. Compared to RBNN. BPNN has fewer neurons, the number of neurons in the hidden layer are eight and four for the $E R E$ and $Q$ networks, respectively. Details of the networks used are listed in Table 6. The spread constant used for RBNN and the error goal of the training data is also given in this table. The spread constant values are selected from the region where the performance of the network is consistent with the variation of spread constant (Figure 15 and Figure 16). The error goal, in the case of solverb, is selected based on the network with the best performance for the ideal spread constant. (Figure 17).

The error in predicting the values of the objective function by different schemes is given in Table 7. Several observations can be readily made.

(1) Both NNs performs better than the RSM for this data set.

(2) Both solverbe and solverb are of comparable performance. 
(3) The BPNN helps generate smaller networks and hence performs at par in comparison to RBNN

(4) The cubic polynomial is more accurate than the quadratic one.

The various models generated are compared with test data shown in Figure 18 and Figure 19. The curves representing the NN predictions are closer to the data obtained from the injector model than the RS thereby demonstrating that NN models are able to predict better than the RS. BPNN performs as well as RBNN but tends to be flat. Due to its lower order, the quadratic polynomial is tlat. The cubic polynomial is able to perform better than quadratic.

\subsubsection{Polynomial-based RSM for Design Optimization}

This case study is used to perform a complete comparative study between polynomial and NNbased RSM. The comparison is carried out in three ways. Firstly, the predictive capabilities of the different models are compared. Secondly, $\mathrm{NN}$ is used to increase the population of the design space, which is then used for mapping by polynomial-based RSM. Thirdly, polynomials and NN are used individually to represent the design space and help in the optimization of the design.

An optimization was done for three different ranges of the independent variables using the quadratic tit. The three cases analyzed differ only in the constraints implemented on the design parameters.

The constraints are

Case $1: 4 \leq O / F \leq 6,4 \leq V / N_{0} \leq 6, L_{c o m b} \leq 7$.

Case $2: 4 \leq O / F \leq 6,5 \leq V, N_{n} \leq 7, L_{c o m b} \leq 7$,

Case $3: 4 \leq O / F \leq 6,6 \leq V / N_{n} \leq 8, L_{c u m b} \leq 7$

The optimization is repeated using the cubic fits. The combinations of weighting factors for $E R E$, $s$, and for $Q, t$, are selected as $(1,10),(1,1)$ and $(10,1)$ for these three cases. The optimum has been evaluated and tabulated for each case, as detailed in Table 8-Table 10. In this effort, injector element optimization means maximizing the performance, while minimizing heat flux and chamber length. The optimum value for $V / N$, obtained on the cubic response surface is quite different than that found on the quadratic surface for some cases (these particular cases are noted in bold in Table 8-10). Also, for selected cases where there are discrepancies between the quadratic and cubic results, the exact values from the 
injector model have been included in parentheses in the tables for comparison. In these cases, the cubic fit more closely matches the exact data than does the quadratic fit. Sample results for $E R E$ plotted in Figure $20 \mathrm{a}$ clearly show the data is better fit by the cubic surface for the case shown. Figure $20 \mathrm{~b}$ shows that the response surface predicted by cubic fit for $Q$ has a noticeable dip that is completely missed by the quadratic fit. This discrepancy results in the optimum for the cubic fit being considerably lower than that for the quadratic surface. The prediction trom cubic fit agrees well with the exact data, which also has a dip for this specific case.

The injector model was also used to produce additional design points to assess the capability of the different response surfaces to match the exact data. In Figure $21 \mathrm{a}$ and Figure 22a, the actual data obtained from the injector model for all the design points has been shown. The cubic and quadratic response surfaces obtained based on the original data is also shown. The RMS error for predicting the new $E R E$ data is 0.270 and 0.205 for the quadratic and cubic surfaces, respectively. For $Q$, it is 0.025 and 0.016 for the quadratic and cubic surfaces, respectively. Again. the performance of the cubic surface is superior to that of the quadratic surface.

\subsubsection{Radial Basis Neural Networks (RBNN)}

Radial Basis Neural Networks are trained by both Solverbe and Solverb tor each injector design response, $E R E$ and $Q$. using the original data set of 45 design points. Solverbe trained the network for $E R E$ with an error to the order of $10^{-13}$. The network trained by Solverbe for $Q$ has an error on the order of $10^{-16}$. Both networks represent the respective design spaces essentially exactly. Solverb, with an error goal of 0.001 , trained networks for both responses to represent the original data set adequately. Since the size of the data set considered for training the network is fairly modest, the number of neurons generated by solverbe is also small. Solverb would have been suited better for a larger data set where a reduction in the number of neurons might have appreciably reduced the computation time. The networks trained using Solverbe have been used for this article. The ability of the RBNN to tit the design data and to generate additional data for constructing a more accurate response surface is discussed in the following sections. 


\section{(i) Comparison between Solverbe and Solverb}

Since Solverbe trains with the same number of neurons ( 45 in this case) as data points, as seen above, it fits the training data set with negligible error. However, it can also create erratic behavior since it makes no attempt to filter noise generated by excess neurons in the network. Solverb, on the other hand, tends to reduce the potential for noise by controlling the number of neurons in the network. Table II shows that in the present article, for the spread constant value of 1.00 . Solverb performs slightly better than Solverbe based on the nominal error measure. However, when judged by the level of errors associated, both RBNNs are satisfactory from a practical standpoint. As expected, Solverb uses fewer neurons than Solverbe; in this case three less. It should be noted that, as investigated in detail by Papila et al. [49], the relative performance between Solverb and Solverbe is case dependent.

\section{(ii) Comparison of RBNN Predictions with Polynomial Based RSM}

Figure $21 \mathrm{~b}$ and Figure $22 \mathrm{~b}$ show that the RBNN trained by Solverbe is able to more accurately generate additional design data than either quadratic or cubic polynomial (shown for comparison in Figure

$21 \mathrm{a}$ and Figure 22a). In Figure $21 \mathrm{a}$, the ERE surface trained with the original data set is shown. The 10 extra design points calculated with the injector model for $V N_{o}$ of 5.00 and 7.00 are shown. The ability of the RB.N to accurately generate new design data can be seen by comparing the fit for ERE in Figure $21 \mathrm{~b}$ to that for the polynomials in Figure 21a. RBNN trains the network with more flexibility and learns the data trend, whereas polynomials provide only an approximate fit on the given data. Regarding the RMS error, $\sigma$. for $E R E$, it is 0.152 for RBNN predictions as compared to the values of 0.270 and 0.205 for quadratic and cubic surfaces, respectively. The four extra design points generated for $Q$, also at $V / V_{o}$ of 5.00 and 7.00 are shown compared to the polynomial surface in Figure $22 \mathrm{~b}$ and compared to the RBNN surface in Figure 22b. The RMS error in the case of $Q$ is 0.022 for RBNN as compared to 0.025 and 0.016 for quadratic and cubic surfaces respectively. Here the performance of the RBNN is better than the quadratic but slightly poorer than the cubic fit. Examination of Table 11 indicates it may be possible that using Solverb with a spread constant of 1.05 could further reduce the RMS for $Q$. However, the errors for $Q$ are low enough that turther reduction may not be practical. 


\subsubsection{RBNN-Enhanced Polynomial Based Response Surface}

Additional design points generated by the RBNN are added to the original data set to form the enhanced data set. This enhanced data set is used for further analysis to evaluate the performance of the RSM with the larger number of design points. The enhanced data set for $E R E$ has 15 points from the injector model and 10 from the RBNN, for a total of 25 points. The enhanced data set for $Q$ has 9 points from the injector model and 4 from the RBNN, for a total of 13 points. The entire optimization analysis was redone with the enhanced data set. On this enhanced data set, the full quadratic response surface seems already appropriately constructed and invoking the statistical analysis generates no reduced model. With the added data in the enhanced data set, it is now possible to obtain a fit for $E R E$ that is $4^{\text {th }}$ order in $V / N_{o}$ and $4^{\text {th }}$ order in $L_{\text {comb }} . Q$ can now be fit with a cubic in $V / V_{o}$ and a quadratic in $O / F$. This is now possible since a combination of 3 different values of O/F, 5 different values of $V / N_{o}$ and 5 different values of $L_{t}, m b$ are available.

\section{(i) Comparison of Fits with the Original Response Surfaces}

Comparison of the enhanced response surfaces with the original response surfaces indicates that the extra data produced with the RBNN generally improves the quality of the curve fit. The adjusted RiMS error for $E R E$ on the original set is 0.211 and 0.083 for quadratic and cubic fits, respectively. On the enhanced data set, it is 0.179 and 0.100 for the quadratic and cubic fits, respectively. The slight increase in the error in the case of the cubic fit may be due to noise related to the over-sensitivity of the polynomial. However, this phenomenon may reflect the fact that the level of the RMS is low enough in either case so that no further improvement is accomplished. The adjusted RMS error for $Q$ with the original set is 0.039 and 0.040 for the quadratic and cubic fits, respectively. On the enhanced set it was 0.027 and 0.026 for the quadratic and cubic, respectively. With the exception of the cubic fit for $E R E$, the fits from the enhanced surface are improved over those from the original surface. Also, when optimum design points are examined, there is less difference between the quadratic and cubic tits on the enhanced surfaces than there is on the original surfaces. 


\section{(ii) Comparison of Optimal Design Points}

The analysis for the three cases of optimization over the same three ranges of independent variables has been re-done. The results of the optimization on surfaces generated from the enhanced data set are tabulated in Table 12-Table 14. The predicted optimal design points using cubic and quadratic fits are generally close to each other. They are closer to each other on the enhanced data set than on the surfaces generated using the original data set. One case where the cubic and quadratic optimum points are somewhat different is analyzed further. The results shown in Figure 23 confirm the optimum value of velocity ratio on the quadratic fit to be lower than the cubic fit in this case. Given the weightings of 1.0 for $E R E$ and 10.0 for $Q$, the optimizer has selects the minimum of $Q$ for both fits. Since the curves exhibit different minimum points, the weightings force the selection of different optimum points. As already discussed, for the polynomial fits on the RBNN-enhanced data sets, the errors of both quadratic and cubic polynomials are more comparable than in the original analysis. At the upper limit of the design space for combustor length, the $E R E$ curves tend to flatten out. This causes some difficulty in locating the optimum and may cause more noticeable differences between the different polynomials. However, different optimal designs selected by different polynomials under such a circumstance are not important since these yield very similar injector performance.

The optimum solution obtained from various schemes is shown in Table 15 and Figure 24 and Figure 25. The aim is to maximize $E R E$ and minimize $Q$. The trend of the ubjective functions in the design space is monotonic and hence every model is able to select identical optimum design for the given constraints. The flatness of the quadratic polynomial results in less accurate values of the objective function for the optimum design. The cubic polynomial, while more flexible than quadratic, is not consistently better in predicting the optimal design point. For example, a $V / N_{v}$ constraint of 4 , the quadratic polynomial is more accurate but for higher values of $V N_{1,}$ the cubic polynomial is more accurate. In contrast, the NN models are able to perform well. Since the optimum design happens to be the same as one of the training points, solverbe is able to predict the values of the objective function accurately. Solverb performs equally well, thereby showing the capability of performance with fewer neurons. Performance of BPNN is not as satisfactory as suggested in Table 7. For lower constraints of $V, V_{i,}$, it performs poorly but for higher values of $V / N_{b}$ it is good. This may be due to the selection of fewer neurons in the hidden layers of the networks. 
Overall, it is still better than the polynomial-based RSM and demonstrates the flexibility of N.V over polynomials.

As stated by Papila et al.[49], when it comes to choosing between NN and polynomials, polynomials are easy to compute. The number of coefficients might be numerous but the linearity of the system expedites the process of coefficient evaluations. This is also the reason RBNV train fast. On the other hand, the weights of BPNN are evaluated through a nonlinear optimization. which slows the training process. Of all the NN presented here, the one designed with the help of solverbe is the fastest to train since the values of the weights are set to values of the input dependent variables. Solverb trains with the addition of one neuron at a time with weights similar to the input and hence is slower.

\subsection{IMPINGEMENT INJECTOR ELEMENT}

\subsubsection{Polynomial Fits}

In Tucker et al. [64], method $i$ uses the polynomial based RSM to find optimal values of $E R E, Q_{w}$, $Q_{i n j}, W_{\text {ret }}$ and $C_{\text {rel }}$ for acceptable values of $\Delta P_{0,}, \Delta P_{j}, L_{c o m b}$ and $\alpha$ The approach of RSM is to perform a series of experiments, or numerical analyses, for a prescribed set of design points, and to construct a response surface of the measured quantity over the design space. In the present context, the five responses of interest are ERE, $Q_{w}, Q_{n j}, W_{r e l}$ and $C_{r i}$. The design space consists of the set of relevant design variables $\Delta P_{o}, \Delta P_{t}, L_{c o m i b}$ and $\alpha$

\section{(i) Individual Polynomial Models}

When the JMP software is used to analyze the 163 design points, five individual tull response surfaces for the variables in the design space are approximated by quadratic polynomials that contain 15 terms each. Using the t-statistics approach noted above and detailed in Tucker et al. [64], unnecessary terms in each equation can be eliminated to give the reduced quadratic surfaces $\mathrm{A}$ survey of the reduced response surfaces indicates that the equations reflect the functionality used to construct the models for the dependent variables. 


\section{(ii) Joint Response Surfaces}

In the current article, it is desirable to attempt to maximize $E R E$ and while simultaneously minimizing $Q_{w}, Q_{i n j}, W_{r e l}$ and $C_{r e l}$. Theretore composite response surface for the present case is given by:

$$
D=\left(d_{E R E} d_{Q_{w}} d_{Q_{m j}} d_{w_{r e i}} d_{C_{m i}}\right)^{1 / 5}
$$

\subsubsection{Optimization Results \& Discussion}

Three sets of results are presented below to demonstrate the capability of method $i$ for the current injector design. These three examples illustrate the effect of each variable on the optimum design, the trade-offs between life and performance issues, and the effect on the design of extracting the last increment of performance.

\section{(i) Effect of Each Variable on the Design Using Original Constraints \& Equal Weights}

The results in this section were obtained by building the joint response surface with the addition of one dependent variable at a time. The results are shown in Table 16. Since current non-optimizer based design methods yield high-performing injector elements, simply maximizing the $E R E$ is not a challenge. Accordingly, the initial results (Case 1) are ubtained with a joint $E R E$ and $Q_{w}$ response surface. The results in Case 2 have the impingement height added. Case 3 adds the relative chamber weight and the relative cost is added in Case 4 . All results are obtained using the original independent variable constraints and all dependent variables have equal weights of one. The results for Case $\$ show that $E R E$ is at its maximum and $Q_{w}$ is very near its minimum desirability limit. Minimizing $Q_{w}$ requires a small $\Delta P_{f}$ relative to $\Delta P_{o}$ as evidenced by the values of 100 psi and 183 psi, respectively. Maximum ERE values are found at the longest chamber length, $L_{\text {rom }}=8$ inches. Even with the relatively high value of 183 psi for $\Delta P_{0}$ and low value of $\Delta P$, of $100 \mathrm{psi}, E R E$ is maximized to $99.9 \%$ with an impingement half-angle of $33.1^{\circ}$.

Addition of the impingement height to Case 2 to model the injector face heat flux, $Q_{i n j}$, forces $\alpha$ lower to increase $H_{i m p i n g e}$ and decrease $Q_{i n j}$. This decrease in the radial component of the fuel momentum has an adverse affect on $E R E$. This effect is mitigated to a degree by increasing the $\mathcal{A}_{f}$ by 32 psi to 132 psi. 
$E R E$ is still reduced by $1.6 \%$. Also, the increase in $\mathcal{A}$. causes increased penetration of the fuel jet, which results in a slightly higher $Q_{n}$.

Case 3 adds the relative combustor weight to the list of dependent variables modeled. Since $W_{\text {ret }}$ is only a function of $L_{\text {comb }}$, minimizing $W_{\text {rel }}$ shortens the combustor length from 8 to 6.6 inches. The shorter $L_{c o m b}$ tends to lower $E R E$. This effect is offset to a large degree by increases in $\Delta P_{j}$ and $\alpha$ both of which increase the radial component of the fuel momentum. The increase in $\Delta P$, also causes a slight increase in $Q_{w}$. The increase in $\alpha$ causes a significant decrease in $H_{\text {impinge }}$, which increases the injector face heat flux. Finally, the relative cost of the injector is added in Case 5. Since $C_{\text {rel }}$ is only a function of propellant pressure drops, both $\Delta P_{o}$ and $\Delta P_{f}$ are driven to their respective minimum values. This and a slight increase in $\alpha$ allow ERE to be maintained at $98 \%$, even with a slight decrease in $L_{c o m b}$. The largest effect of this fairly dramatic decrease in propellant pressure drops is on $Q_{w}$. Even though the values for $\Delta P_{1}$, and $\Delta P_{j}$ fell,

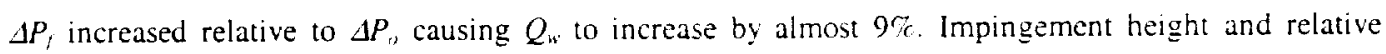
combustor weight are essentially unchanged.

Although several of the variables included in this exercise are qualitative, an important conclusion can still be drawn. The sequential addition of dependent variables to an existing design results in changes to both the independent and dependent variables in the existing design. The direction and magnitude of these changes depends on the sensitivity of the variables, but the changes may well be signiticant. The design in Case 4 is quite different that the one in Case 1 . Consideration of a larger design space results in a different design-the sooner the additional variables are considered. the more robust the final design will be.

\section{(ii) Emphasis on Life \& Performance Issues Using Original Constraints \& Unequal Weights}

The purpose of this section is to illustrate the effect of emphasizing certain design criterion on the optimization process. Method $i$ allows this emphasis via the weights applied to the desirability tunctions in the joint response surface. The results shown in Table 17 facilitate the illustration. The Case 1 (baseline) results are repeated from Case 4 in this table where the entire design space is considered with the original constraints and equal weights for the dependent variables. The results in the Case 2 column are obtained by emphasizing the minimization of the wall and injector face heat tluxes. Desirability functions for both of these variables are given a weight of five. Since lower heat tluxes tend to increase component life, 
weighting these two variables is equivalent to emphasizing a life-type issue in the design. As expected, $\alpha$ is decreased to increase $H_{\text {impinge }}$, thus decreasing $Q_{: n}$. Since the fuel pressure drop is already at the minimum, the oxidizer pressure drop is increased by $58 \%$ to decrease $Q_{w}$. Both of these changes tend to decrease ERE While $E R E$ does decrease, the effect is somewhat mitigated by an increase in $L_{c u m b}$. The increases in $L_{\text {comb }}$ and $\Delta P_{o}$ cause increases in $W_{r e t}$ and $C_{\text {rel }}$, respectively. The emphasis on life extracts the expected penalty on performance. Additionally, for the current model. there are also weight and cost penalties.

The results for Case 3 are obtained by emphasizing maximization of $E R E$ and minimization of $W_{\text {rel }}$ with desirability weightings of five. Increased weighting for these two variables is equivalent to emphasizing a thrust to weight goal for the injector/chamber. The relative chamber length is shortened to lower $W_{r e l} . E R E$ is maximized by increasing the radial momentum of the fuel jet. Both $\Delta P_{f}$ and $\alpha$ are increased to accomplish ERE maximization. As noted earlier, increasing $\mathcal{A} P_{f}$ and $\alpha$ lead to increased wall and injector heat fluxes, respectively. Table 17 indicates that to be the case here. For this case, emphasis on thrust and weight tend to have an adverse affect on both $Q_{*}$ and $Q_{n j}$. Relative cost, for the current model, is not significantly affected.

\section{(iii) Extraction of Last Performance \& Weight Increments (Modified Constraints \& Unequal}

\section{Weights)}

Here, the high marginal cost of realizing the last increment of thrust to weight is shown. This section illustrates the capability to modily the constraints on the independent variables and use unequal weights on the dependent variables at the same time. The results for Case 3 in Table 17 are carried over to Case 1 in Table 18 as the baseline for this example. Here the original constraints are used but increased weights have been applied to emphasize $E R E$ and $w_{\text {rei }}$. Cases 2 and 3 modify the constraints on the propellant pressure drops, raising the minimum pressure drop from 100 psi to 150 psi. For Case 2, both $\mathcal{A P}_{w}$ and $\Delta P_{f}$ are now at the minimum level for the modified constraints. $L_{r i m b}$ is increased slightly to maintain $E R E$. The decrease of $\Delta P_{l}$ relative to $\Delta P_{1}$ causes a decrease in $Q_{w}$. The slightly higher-pressure drops also cause $C_{r t}$ to increase somewhat. Other variables are not changed appreciably.

For Case 3, ERE and $W_{r e i}$ are further emphasized by increasing their desirability weights to 10 while decreasing the other weights to $0.1 . L_{c i m m}$ is shortened to respond to the increased emphasis on weight 
minimization. Maintaining the high level of $E R E$ requires large increases in $\Delta P_{f}$ and $\alpha$ to increase the radial component of the fuel jet momentum. The increase in $\Delta P_{f}$ causes over-penetration of the fuel jet, which results in an increase in wall heat flux. The large increase in $\alpha$ yields the expected decrease in $H_{\text {impinge }}$ which increases the injector face heat flux. The additional emphasis on $E R E$ and $C_{r e t}$ yields essentially no increase in $E R E$ in this range of $\triangle P$, although a small weight savings is seen. These marginal improvements are offset by fairly large increases in $C_{r e i}$ and $Q_{i n j}$.

\subsection{SWIRL CO-AXIAL INJECTOR ELEMENT}

Two sets of results are presented below to demonstrate the capability and flexibility of method $i$ for the current injector design. These examples illustrate the effect of each variable on the optimum design and the trade-offs between life and performance issues.

\subsubsection{Effect Of Each Variable On Element Design}

The results in this section were obtained by building the joint response surface with the addition of one dependent variable at a time. The results are shown in Table 19. Case 1 seeks the maximum performance without regard to the effect on the other dependent variables. ERE is a fairly strong function of $L_{t r m b}-$-longer chamber lengths allow more residence time for the propellant to mix and burn. The effect of $\Theta$ on $E R E$ is strongest at low values of $\Theta . E R E$ increases with increasing $\Theta$ until about $\Theta=80^{\circ}$ and then fall off slightly due to the competing influences noted earlier. These competing influences also cause the effect of both pressure drops on $E R E$ to be somewhat flat, although since $\Delta P_{0}$ affects more variables, its influence is slightly stronger. Maximum performance is found at high values of $\Delta P_{\alpha}, \Theta$, and $L_{\text {cumb }}$ and at low values of $\Delta P_{j}$. This trend is consistent with other works for similar injector elements. The predicted optimal value of 98.5 is indeed the highest predicted by this model.

The objective of Case 2 is to simultaneously maximize $E R E$ and the minimize $Q_{w}$ Table 19 shows that the exact same design point was chosen as for Case 1. Usually, the design, which yields the maximum $E R E$, also produces a high wall heat flux. That is not the case here; this issue has already been noted. The minimum $Q_{w}$ is found in the region of high $\Delta P_{n}$ and low $\Delta P_{f}$. In this area, $Q_{w}$ is almost independent of $\Theta$. 
Hence, the minimum $Q_{w}$ can still be found for a high value of $\Theta$ required to maximize $E R E$. It should be noted that in the low $\Delta P_{o}$, high $\Delta P_{f}$ region, $Q_{\star}$ is a function of $\Theta$. Here, as $\Theta$ is increased, $Q_{w}$ increases since the larger swirl angle forces $d_{o}$ to increase and thus decrease $V_{r}$. In the Calhoon et al. [3] model, this reduction in $\mathrm{GO}_{2}$ momentum causes an increase in $Q_{w}$.

The requirement to minimize $Q_{1 n j}$ is added in Case 3 . In order to minimize $Q_{2 n j}$, the swirl angle is decreased from $81^{\circ}$ to $37^{\circ}$, thus reducing the injector face heat flux by approximately a factor of 3 . This decrease in $\Theta$ also lowers $E R E$ which forces use of a longer chamber to offset some of the loss. Still, $E R E$ is reduced by over one percent.

Case 4 considers the desire to minimize the chamber weight, $W_{\text {ret, }}$ in addition to maximizing $E R E$ and minimizing $Q_{w}$ and $Q_{i n \text {. }}$. Since $W_{r e l}$ depends only on $L_{c o m b}$, the chamber length is shortened by over half. The weight is reduced, but so is ERE. To mitigate the adverse effect on $E R E, \Theta$ is increased by almost $10^{\circ}$, simultaneously increasing $Q_{i n}$. ERE drops again by over a percent, while $Q_{w}$ remains constant.

Finally, minimizing the injector cost. $C_{e_{i},}$ is added in Case 5. Decreasing each pressure drop approximately a factor of 2 lowers the relative injector cost. Decreasing $\Delta P_{f}$, results in a larger fuel gap and decreasing $\Delta P_{o}$ allows for a larger swirl slot. These factors combine to lower the cost by almost $10 \%$.

Although several of the variables included in this exercise are qualitative, an important conclusion can still be drawn. The sequential addition of dependent variables to an existing design results in changes to independent and dependent variables in the existing design. The direction and magnitude of these changes depends on the sensitivity of the variables, but the changes may well be significant. The design in Case 5 is quite different that the one in Case 1. Consideration of a larger design space results in a different designthe sooner the additional variables are considered, the more robust the final design.

\subsubsection{Emphasis on Life and Performance Issues}

Method $i$ allows this emphasis via the weights applied to the desirability functions in the joint response surface. The set of results shown in Table 20 facilitate the illustration. The baseline results Table 20 (repeated from Case 5 in Table 19) consider the entire design space using the original constraints and equal weights for the dependent variables. The results are obtained by emphasizing the minimization of the 
wall and injector face heat fluxes for Case 1. Desirability functions for both of these variables are given increased weights (5 and 10, respectively). Since lower heat fluxes tend to increase component life. weighting these two variables is equivalent to emphasizing a life-type issue in the design. Since $Q_{*}$ is already at its minimum value, it remains fixed. As expected, $\Theta$ is decreased, which decreases the value of $Q_{i n j}$ by almost $35 \%$. The lower value of $\Theta$ also produces a lower $E R E$. Both propellant pressure drops and the combustor length are increased to mitigate the drop in $E R E$. The increases in $L_{c o m i}$ and $A P$, cause increases in $W_{r e l}$ and $C_{\text {rel }}$, respectively. The emphasis on life extracts the expected penalty on performance. Additionally, for the current model, there are also slight weight and cost penalties.

The results for Case 2 are obtained by emphasizing maximization of $E R E$ and minimization of $W_{\text {rel }}$ with desirability weightings of 10 and 5 , respectively. Increased weighting for these two variables is equivalent to emphasizing a thrust to weight goal for the injector/chamber. The relative chamber length is shortened to slightly lower $W_{\text {rel. }}$ ERE is maximized by increasing the $\mathrm{GO}_{2}$ swirl angle by a factor of almost 2.5 and also increasing $\triangle P$, by over $35 \%$. The value of $E R E$ rises by over one percent. As noted earlier, increasing $\Theta$ leads to increased injector heat flux. For this case, emphasis on thrust and weight tends to have an adverse affect on $Q_{i n j}$. Relative cost, for the current model, is also increased significantly. Performance and weight trends for the swirl and impinging injector elements are shown in Figure 26. Figure 27 shows the heat flux and cost trends for the swirl and impinging injector elements.

\subsection{SUPERSONIC TURBINE FOR REUSABLE LAUNCH VEHICLES}

\subsubsection{Polynomial-Based RSM Results for 1-, 2- and 3-Stage Turbines}

There are 28-unknown coefficients needed for constructing the $2^{\text {nd }}$-order response surface for the single-stage case, 78 for the 2-stage and 136 for the 3-stage case. Different starting points are tried to avoid local maximum and the optimum values of $\eta, W$ and $\Delta p a y$ with the corresponding design parameters are determined. The results shown are comparable with the corresponding Meanline runs with the highest error of $5 \%$ for $\Delta$ pay for single-stage turbine. The percentage error is increased to $13.5 \%$ for $\Delta$ pay for 2 -stage turbine and to $14.6 \%$ for the 3-stage turbine for $\Delta$ pay indicating that the accuracies of the response surfaces constructed are poor for the 2- and 3-stage. 
Papila et al. [50] have reduced the size of the parameter space by $80 \%$ in each coordinates, based on the optimal design selected in the original design space, to improve the accuracy of the response surfaces for these cases. The intention is to improve the fidelity of the response surface. With these refined designed spaces, substantial improvement of the response surface fit accuracy is observed for both cases by Papila et al. [50].

Based on the results obtained, the following observations can be made:

To ascertain required predictive capability of the RSM, a two-level domain refinement strategy has been adopted by Papila et al. [50]. The accuracy of the predicted optimal design points based on this approach is shown to be satisfactory.

(2) According to the results obtained for $\Delta$ pay-based optimization, the 2-stage turbine gives the best $\Delta$ pay result.

(3) As the number of stages increases, it is observed that efficiency improves while the weight increases also but the improvement in efficiency can't compensate the penalty from higher weight.

(4) As shown in Figure 28, the mean diameter, speed, and the exit blade area exhibit distinct trends. Specifically, the diameter decreases, speed increases, and annulus area decreases with increasing number of stages. It is interesting to observe that none of these design parameters are toward the limiting values, indicating that the optimal designs result from compromises between competing parametric trends. For such cases, a formal optimizer such as the present response surface method is very useful.

Table 21 gives a summary of the optimization results for 1-, 2- and 3-stage turbines for $\Delta$ paybased optimization.

\subsubsection{Higher Order Polynomials and NN-Based RS.M for Single-Stage Turbine}

The generation of polynomial-based RS model and the training of the $\mathrm{VN}$ are done with 76 design points of the single-stage turbine. The analysis was initially done without the constraints and then with the constraints on $(A N)^{2}$ and $V_{\text {putch }}$

A quadratic polynomial model was initially generated. Then, cubic terms were included. Cubic terms that are products of three different variables were included because of the number of data available and the 
number of levels being three. The trend of the design data also suggests the presence of some of these terms. Therefore, the initial cubic equation has 45 terms. Reduced third order polynomial model for $\eta$ and $W$ were selected based on the relative performances of different polynomials obtained by removing terms from the initial cubic equation based on t-statistics. The cubic equation was selected based on the evaluated value of $\sigma_{a}$ and $\sigma$. Table 22 suggests that the reduced cubic polynomial is better than the quadratic polynomial since $\sigma_{a}$ is better for the former. The value of $\sigma$ is comparable.

When constructing the NN-based response surface, the design parameters of the NN should be selected carefully since the selection of the design parameters determines the learning characteristics of the NN. For the single-stage supersonic turbine case, the variation of $\sigma$ with respect to the only design parameter of solverbe network, spread constant, is plotted in Figure 29 for both objective functions of $\eta$ and $W$. Figure 30 shows that for low values of spread constant, the NN has a poor performance. As the spread constant increases $\sigma$ asymptotically decreases. Therefore, the appropriate spread constant is selected from the region where the performance of the network is relatively consistent. Figure 31 shows the influence of error goal on the network performance. Unlike the case of injector (Figure 17), a more stringent error goal for the training data does not necessarily result in better predictive capability against the test data for the single-stage turbine.

The networks designed with solverb have 37 and 75 neurons for $\eta$ and $W$, respectively in the hidden layer, while those designed with solverbe has 76 neurons each. The BPNN uses significantly less number of neurons by generating networks with five and 60 neurons for $\eta$ and $W$, respectively, in a single hidden layer. The NN architectures chosen are listed in Table 23.

The accuracy of the various models is tested with the 18 additional available data and the error is shown in Table 24. Solverbe has a poor prediction for $\eta$, which might be due to over fitting, but performs well for $W$. Solverb is most consistent among all methods evaluated.

The optimum solutions subjected to the constraints, of $(A N)^{2}$ limited to less than 1.132 (normalized with baseline value) and $V_{\text {puth }}$ is limited to less than 1.148 (normalized with baseline value), are presented in Table 25. Since $(A N)^{2}$ is proportional to the product of square of $R P M$ and $A_{\text {unn }}$, and $V_{\text {puch }}$ is proportional to $D$ times $R P M$. no NN or polynomial-based RSM is generated for them. By comparing the predicted optimal design by the various methods, one observes that solverbe and BPNN yield noticeably 
larger error in $\eta$ and $W$, respectively. Solverb and the response surface are more consistent with both $\eta$ and $W$. Judged by the error in predicting $4 p a y$, it seems that the polynomial based RSM is most accurate. However, since the real goal is to maximize $\Delta p a y$, it is important to note that the actual value of $\Delta p a y$ for the optimal design chosen by the RSM is the worst.

From a design perspective, it is interesting to understand the impact of the constraints from $A_{u n n}$ and $V_{\text {pich }}$ on the optimal turbine parameters. Such an assessment is offered in Figure 32 and 33. As D. RPM and $A_{a n n}$ decrease, $\eta, W, V_{\text {pich }}, A N^{2}$ and $\Delta p a y$ decrease. $C_{b}$ and $C_{v}$ are almost constant over the design space and they do not have any noticeable effect on the objective functions and constraints. In the case of $C v$, the BPNN shows a small perturbation for the analysis with the constraint. This might be due to the mapping of some noise by BPNN. Otherwise it is unaffected by the inclusion of the constraints. The optimum stage reaction, $K_{r}$, is zero implying that the optimum design should be an impulse turbine.

\subsubsection{Orthogonal Arrays For 2-Stage Turbine}

Although the majority of the work is based on the face centered composite design approach (FCCD), orthogonal arrays (OA) are constructed by Papila et al. [50] to investigate the efficiency of orthogonal array designs in representing the design space for 2-stage turbine. A set of 249 design points is selected using orthogonal arrays. Table 26 shows the comparison of the quality of the second-order response surfaces generated for $\eta, W$ and $\Delta$ pay by using 1990-data generated by face centered composite design and 249-data selected by orthogonal array method.

The above table illustrates that the fidelity of the response surface generated for design space of 249 data, based on orthogonal arrays, are comparable with that of 1990 data based on the face centered criterion. The response surface models are also assessed by using 78-test data to determine the predictive accuracy of these models. Table 27 presents that the testing adjusted rms-errors of response surfaces generated are $1.65 \%$ for $\eta$ and $0.96 \%$ for $W$ using 249 -data. and $1.67 \%$ for $\eta$ and $1.21 \%$ for $W$ using 1990 data.

When these results are compared with the results of 1990-data and it is observed that the optimum $\eta, W$ and $\Delta p a y$ are largely consistent However, it is also observed from Figure 34 which shows the 
comparison of the design variables for optimization based on ( $\Delta p a y)$, some of the design variables are different even though optimum $\eta, W$ and $\Delta p a y$ are consistent. This shows that there are multiple points in the design space, which yield comparable performance. Nevertheless, it remains true that the two-stage turbine is most suitable from a payload point of view.

\subsubsection{NN-Based RSM for 2-Stage Turbine}

In order to find the optimum RBNN design for the design of the two-stage turbine design, the effect of the spread constant $(s c)$ on the network training error is determined. Figure 35 and Figure 36 show the variation of solverbe network error, $\sigma$, with respect to spread constant for the NN designed for FCCD and OA data. The optimum spread constant is determined as 3.2 for 1990-training data (FCCD) from Figure 35 and 4.3 for 249-data (OA) from Figure 36. In spite of the fact that, the spread constants larger than 3 give reasonable training rms-errors $\left(\sigma_{u}\right)$, less than $0.1 \%$ for all networks designed for refined space with 249-data as shown in Figure 37, sc=4.3 value is used for these cases for consistency.

After constructing the $\mathrm{NN}$-based response surface, the $\mathrm{NN}$ model is tested by using 78-test data selected along the main diagonal of the design space to determine the predictive accuracy of these models. Table 28 presents that the prediction rms-errors $(\sigma)$ of response surfaces generated by for second order polynomial are $1.65 \%$ for $\eta$ and $0.96 \%$ for $W$ using 249 -data, and $1.67 \%$ for $\eta$ and $1.21 \%$ for $W$ using 1990-data. Table 28 also presents that the prediction rms-errors of response surfaces generated by solverbe RBNN are $1.36 \%$ for $\eta$ and $1.30 \%$ for $W$, and $2.26 \%$ for $\eta$ and $1.56 \%$ for $W$ using 249 -data.

Figure 38 summarizes fitting/training and testing results of RBNN and polynomial-based $\triangle$ pay approximations for 2-stage turbine. The efficiency of the multi-level RSM approach can be observed by comparing the original and refined design space plots. From these plots, it is also possible to observe that more accurate training is possible with RBNN but testing or prediction accuracies of the RBNN and polynomial-based approximations are quite comparable. 


\subsection{TURBULENT PLANAR DIFFUSER}

\subsubsection{Polynomial Fits}

Based on the D-optimal set of 35 design points selected, the 21 regressors of a full quadratic polynomial were fitted resulting in a moderate $R_{a}{ }^{2}$-value of 0:810.A backward elimination of regressor terms subsequently led to the removal of five terms and an increase of $R_{a}{ }^{2}$ to 0.848 . The lower values of $R_{a}{ }^{2}$, in comparison to the two-design-variable case, reflect the increased difficulties in obtaining a good fit when moving to higher-dimensional response surfaces. Data on the backward elimination steps are given in Table 29, which apart from $R^{2}$ and $R_{a}{ }^{2}$ holds the minimum t-statistic and the number of uncertain terms with $\left|t_{0}\right|<2.0$ remaining in the model. From the t-statistics information, it appears that the backward elimination improved the accuracy of remaining terms.

The next step performed was to investigate whether the 35 applied observations included outliers A common (but not necessarily true) assumption, which enables the statistical treatment of observations, is that errors are independently and identically distributed according to a normal distribution with mean zero and variance. Thus, the distribution of response surface errors was plotted and compared to a normal distribution, with which it is expected to correspond well. From the histogram plot of the error distribution, see Figure 39, it did not seem that there are any outliers. Four arbitrary points away from sampling points were picked to test the prediction accuracy of the polynomial-based RSM. Table 30 compares CFD-results and polynomial approximations with and without backward elimination of terms.

Again, the predictions of the response surface appear reliable, apart from at the last control point. This point is, however, well in the non-monotonic region, so that the approximation relies on an extrapolation, which was never intended. The reduced approximation model comes closer to the CFDresults for two out of the three meaningful test points.

\subsubsection{Numerical Noise}

While noisy data from laboratory experiments is a generally accepted fact, the presence of noise in numerical simulations seems much less recognized. Due to the complex numerical modeling techniques of CFD, the exact origins of noisy responses may be difficult to pinpoint. but factors such as turbulence 
models, incomplete convergence, and the discretization itself are certainly influential. Here, the presence of numerical noise has been investigated. The problem of non- smooth or noisy objective functions has previously been addressed by Giunta et al. [17], who found RS approximations-based optimization to perform very robustly under such circumstances, especially when point selection is based on design of experiment techniques, such as D-optimal designs.

Limitations of the software used were felt during the application of a wall shape parameterization in the investigation of noise. A $B$-spline curve with two free control points was used. Again, it was observed that the objective function oscillated due to numerical noise, but the amplitude was small. To make the noise more apparent, it was therefore necessary to refine the subdivision of the discretized line and reduce its length to $20 \%$ of the initial, so that the line spans from $(0.3,0.6)$ to $(0.302,0.602)$. This yielded the noisy response patterns shown in Figure 40. The two topmost curves in this figure were determined using a relatively tight convergence criterion, and two different convection schemes - a standard first-order upwind differencing scheme (UDS) and a second-order upwind differencing scheme (SUDS). The use of different differencing schemes was carried out to estimate whether numerical diffusion does significantly dampen the generation of noise. As discussed in Madsen et al. [33], two different CFD codes were adopted, and one seems less forgiving, in the sense that it predicts a stronger tendency for flow separation. This could possibly be explained by factors such as numerical diffusion, boundary treatments, and momentum interpolation methods adopted in the two codes.

As expected, switching to a more dissipating differencing scheme (lower order accuracy) yields a smoother response. To further illustrate this issue, one more design line curve is shown in Figure 40, which arose from using a relatively loose, yet still reasonable, convergence criterion (using SUDS). The applied convergence criterion considers summed and normalized (by inlet flux) residuals over the entire mesh, with termination of computations once the maximum is below a certain small value $\varepsilon$. The loose convergence criterion in Figure 40 was $\varepsilon=10^{-3}$, whereas the tight tolerance was $\varepsilon=10^{-5}$. For comparison, a convergence limit of $\varepsilon=10^{-4}$ was applied in the CFD analyses used for response surface modeling. The overall conclusion is that the presence of some numerical noise in CFD-results is practically inevitable, although its magnitude depends on choice of code and modeling techniques. Here, a technique such as polynomialhased RSM can be effective in smoothing out the undesirable tluctuations. 


\subsubsection{Optimum Diffuser Designs}

In the optimum design using B-spline parameterization, both the monotonicity constraint and four out of tive side constraints are active. As already mentioned, the response surface constructed to guarantee wall monotonicity becomes too restrictive. To compensate for this, a one-dimensional search in the direction of the steepest gradient was conducted starting at the optimum design point estimated by RSM

$$
y=y^{*}+\alpha \nabla F
$$

This search is terminated as soon as designs turn non-monotonic, yielding a new optimum point at the edge of the true feasible domain and an increase in the optimum pressure recovery coefficient from 0.7208 to 0.7235 . Figure 41 compares the optimum wall contours determined by RSM using B-splines and polynomial shapes. The optimum B-spline shape compares well to the optimum polynomial one, so it is not surprising that there is no significant gain compared to this case. The largest differences in shape are found in the later part of the expansion, where the shape has less impact on the overall performance, as separation is small in either case. Thus, the close resemblance of optimum inlet shapes is reassuring in terms of the credibility of the optimization algorithm. A CFD-analysis of the five-design-variable optimum design yields a pressure recovery coefficient of 0.7193 , a little below the predicted value, as in the two-designvariable case. The improvement from the two design variable case $(0.7185$ to 0.7193$)$ indicates that there is not much potential for further gains. Furthermore, for comparison, Figure 41 also contains the corresponding wall contour determined using search optimization techniques. The optimum wall shape found by search optimization can be described as truly bell-shaped, without a "plateau" similar to the one found in the results of RSM-optimization. There appears to be a distinct difference in optimum shapes from the two different optimization approaches, which must be ascribed to the combination of optimization accentuating modeling differences and a relatively small scatter in diffuser performances.

Figure 42 highlights the use of a response surface approximation for the optimum shape of a twodimensional diffuser. As illustrated, within the fidelity of the analysis tool, there are often multiple design points that meet the design objectives. It is interesting to note that different diffuser shapes are found to yield essentially the same performance. The response surface model is ideally suitable for such situations. 


\subsection{LOW REYNOLDS NUMBER WING MODEL}

\subsubsection{Polynomial Fits}

For the 3-D wing case, the response is the flight power index, $C_{2}^{3 / 2} / C_{0}$, and the design space consists of design variables maximum camber, $y_{,}$, and wing aspect ratio, AR. Quadratic, cubic and quartic order polynomials are tested for the best approximations for data sets containing 9,15 and 25 simulated data points (See Table A1). The predicted RMS errors are calculated for each of the model and are shown in Table 31. As shown in this table, Model 4 gives the smallest predicted RMS for the cases involving 9 and 15 simulated data points, whereas, Model 12 allows the smallest predicted RMS error for the case involving 25 simulated data points.

\subsubsection{Comparison of Radial-Basis and Back-propagation Networks}

The predictive accuracy of neural networks depends not only on the training data but also on the parameters used to define the network. The best values for these parameters cannot be determined by using only training data, because typically one can obtain very small errors for the training data with a wide range of these parameters. However, the performance of $\mathrm{VN}$ can be examined using test data.

For the radial-basis network, one important issue is to investigate the magnitude of error in the test data to help to select the spread constant. For the back-propagation network, where cost of computation is an issue, the effect of number of neurons on the cost and accuracy should be checked. It was noticed that for the back-propagation network, using four neurons gave a good compromise of accuracy and cost. For the radial-basis network, it was found that the error and the number of iteration required for convergence are extremely sensitive to the value of spread constant. After extensive experimentation, the spread constant was chosen as 1.175 .

For the 3-D wing case, both radial-basis VN and back-propagation networks are applied. In order to be able to make comparisons between these networks, the training time histories are summarized in Table 32 and Table 33. These tables show that both are efficient in the training of 9-simulation, 15 simulation and 25 -simulation training data sets. However, as the data size increases, the back-propagation 
network exhibits a growth rate in terms of the number of epochs, indicating that it is more CPU time intensive for larger data sizes. As far as accuracy is concerned, both networks perform well exhibiting improved predictive capabilities as the number of training points increases from 9 to 25 -simulation for $y_{c}$ interpolations ( Figure 43). For this case, both methods reproduced the original 9 -simulation accurately but both failed to predict accurately the interpolation points at $y_{c}=0.0125,0.025,0.075$ and 0.0875 with the RMS error of the test data of 1.68 for back-propagation network and 1.04 for radial-basis network ( Figure 43a). Figure $43 \mathrm{~b}$ shows that adding 6 new points at $A R=2$ and 4 at $y_{c}=0,0.05$ and 0.1 (15-simulation training data set) does not significantly improve the 6 interpolated values (RMS values of 1.369 for backpropagation network and 1.029 for radial-basis network). However, with the addition of 10 new points at $y_{c}=0.025$ and 0075 at $A R=1,2,3,4$ and 5, (25-simulation training data set) both the back-propagation network and the radial-basis network can accurately capture the overall behavior of the aerodynamic data as shown in Figure 43c. The RMS error now is 0.141 for back-propagation network and 0.106 for radialbasis network. For $A R$ interpolations, the back-propagation network resulted in lower RMS values when compared to the RMS values of radial-basis networks (Figure 44). For the 9-point simulation training data, the RMS of radial-basis network (RMS=11.12) is quite high when compared to the RMS of backpropagation (RMS $=1.172$ ) (Figure $44 \mathrm{a})$. For this case, adding 6 new points at $A R=2$ and 4 at $y_{r}=0,0.05$ and 0.1 significantly improves the RMS value for radial-basis (RMS=0.87) as shown in Figure 44b. With the addition of 10 new points to 15 -simulation data at $y_{c}=0.025$ and 0.075 at $A R=1,2,3,4$ and 5 , the RMS error decreases further to 0.7 for radial-basis networks, and 0.026 for back-propagation (Figure 44c). The results indicate that the back-propagation network is quite accurate for small to modest number of data for the cases investigated and it is also more consistent than that of the radial-basis network. However, as indicated in Table 32 and Table 33. in terms of computing time or epochs, back-propagation network scales unfavorably with respect to the number of data used. In other words. the back-propagation network is competitive for modest data size while the radial-basis network is more effective for larger data size. More information will be presented when the 2-D airfoil case that involves substantially larger data size is discussed. 


\subsubsection{Comparison of Radial-Basis Neural Network and Polynomial-Based Techniques}

For the 3-D wing model, the outputs of the solverb radial-basis NN, along with the results of the polynomial-based technique, are compared for different size of the data. It must be noted that the network parameters used to obtain radial-basis network results are $s c=1.175$ and error goal $=10^{-2}$. Figure 45 illustrates the comparison between the $\mathrm{NN}$ and polynomial-based outputs based on the 9-simulation training data set. For this case, both methods reproduced the original 9-simulation accurately but both failed to predict accurately the interpolation points at $y_{c}=0.025$ and 0.075 with RMS errors at the test data of 1.04 for both the NN and polynomial-based methods. Furthermore, it is seen that the error estimate of 1.116 of Table 31 is a gross underestimate. Note that by the time there are 25 data points, Table 31 predicts an error of 0.659 . The reason for this problem is that RMS error estimates are not reliable when the number of coefficients is close to the number of points ( 7 vs. 9 for this case). In addition, these estimates assume random noise and that underlying function is quadratic. Figure 45 b shows that adding 6 new points $A R=2$ and 4 at $y_{t}=0,0.05$ and 0.1 does not help noticeably to improve the 6 interpolated values (RMS values of 1.029 for both). However, with the addition of 10 new points at $y_{c}=0.025$ and 0.0075 at $A R=1,2,3,4$ and 5 , (25-simulation training data set) both the $\mathrm{NN}$ and polynomial-based techniques accurately capture the overall behavior of the aerodynamic data as shown in Figure $45 \mathrm{c}$. The generalization of the NN with 25 simulation is further assessed by comparing additional interpolated values at different $\mathrm{y}_{\mathrm{c}}$ and $A R$ at $y_{C}=0.0125$ and 0.0875 at $A R=1,2,3,4$ and 5 . The RMS errors now are 0.142 for the polynomial and 0.221 for the $\mathrm{NN}$, which are more in the line with the prediction in Table 31 .

These comparisons illustrate that both neural network and conventional polynomial fitting methods do a good job as the number of points is increased

\section{CONCLUSION AND FUTURE DIRECTIONS}

Recent experiences in utilizing the global optimization methodology, based on polynomial and neural network techniques, for aerodynamics and rocket propulsion components are summarized. Global optimization methods can utilize the information collected from various sources and by different tools. These methods offer multi-criterion optimization. handle the existence of multiple design points and tradeoffs via insight into the entire design space, can easily perform tasks in parallel, and are often effective in 
filtering the noise intrinsic to numerical and experimental data. Another advantage is that these methods do not need to calculate the sensitivity of each design variable locally. The global optimization method can be particularly effective with either a polynomial-based response surface or a neural network when information from different computational, experimental, and analytical sources needs to be assembled. In this article, we present recent experiences in utilizing the global optimization methodology for tasks related to the preliminary design of a supersonic turbine, multi-criterion design of three different types of injector element (shear co-axial, impingement, and swirl co-axial), performance of a low Reynolds number wing, and shape optimization of a turbulent flow diffuser. A successful optimal design often needs to address the issues related to the selection of appropriate training data for constructing the global model, employment of the statistical and testing tools to identify appropriate global models, existence of multiple design selections and related trade-offs, and consideration of noises intrinsic to numerical and experimental data. These issues are discussed. It is seen that the global optimization method can naturally take the confidence level of the data into account, offers a number of designs with comparable performance, and allows designers to make a more informed decision. We have reviewed direct evidences that demonstrate that appropriate selection of design points can significantly reduce the number of data required for constructing the global model. In particular, while the FCCD approach can be effective with modest number of design variables. OA with D-optimal selection criterion seems to be effective when the number of design variables becomes higher. Regarding the relative merits between polynomials and neural networks. based on the results reviewed, we can make the following summary.

1. Higher order polynomials usually perform better than lower order polynomials as they have more flexibility. However, exceptions have been noticed which demands that appropriate statistical measures be taken to determine the best terms to include in an expression.

2. Both NN and polynomial based RSM can pertorm comparably for modest data sizes.

3. Among all the NN configurations, RBNN designed with solverb seems to be more consistent in performance.

4. Radial basis networks, even when designed efficiently with solverb, tend to have many more neurons than a comparable back-propagation with tan-sigmoid or log-sigmoid neurons in the hidden layer. The basic reason for this is the fact that the sigmoid neurons can have outputs over a large region of the 
input space, while radial basis neurons only respond to relatively small regions of the input space. Thus, larger input spaces require more radial basis neurons for training.

5. Configuring a radial basis network often takes less time than that for a back-propagation network because the training process for the former is linear in nature.

6. While the transfer function employed by any neural network is nonlinear in general, RBNN, with the combined feature of thexibility and linear regression is more accurate than BPNN, which requires solution of nonlinear systems.

7. The comparisons demonstrate that for this case there are no significant differences between the NN and polynomial based RSM. The results of polynomial-based methods, though, suggest that when the error is mostly due to modeling rather than noise, the error estimates of the polynomial-based technique can be substantially off.

8. The NN technique has shown the potential of fitting the data much better than the polynomialbased technique. However, this was achieved by using the test data to select the parameters like spread constant of the $\mathrm{NN}$ which appear to greatly affect the predictive accuracy. That is, it was not possible to use only the training data to select the best set of parameters. This indicates that because the NNs do not provide the statistical information given by polynomial-based methods, using both test data and training data is very important in designing the network.

9. With the large number of points, and the high order polynomial, the statistical predictions of the polynomial-based results matched very weil the error at the test data

10. The neural networks, when trained appropriately, can be used to generate additional data to help enhance the data set for constructing the polynomial. Such a combined approach has been demonstrated in Shyy et al. [57] for injector design

11. The criteria for selecting the data base exhibit signiticant impact on the efficiency and effectiveness of the construction of the response surface. For example, effectiveness of using OA to select the database is demonstrated by Papila et al. [50]

12. A multi-level approach can be applied to identity the optimal design points with substantially higher accuracy. 
There are a number of outstanding issues that need to be addressed. In the following, we list several topics that we consider important for future research.

1) Is it possible to develop a comprehensive technique by combining $N N$ and polynomial-based $R S$ techniques to help reduce the required data size for optimization?

Specifically, the work done by Rai and Madavan [51], [52] \& [53], Madavan et al. [31], and Shyy et al. [57] suggests that $\mathrm{NN}$ can be effectively used to supplement the existing training data to help generating a more accurate polynomial. RBNN may lack satisfactory filtering properties in some cases (Papila et al. [49], Vaidyanathan et al. [71]). However, once trained, RBNN can generate additional design data to feed the polynomial-based RSM. Polynomials possess the intrinsic filtering capability. The evaluation of the nature of the fluctuations from the data generated by RBNN, and the investigation into whether polynomials can use the data effectively, is planned. These features have been addressed in this article.

2) What are the keys to develop a more robust and flexible NN configuration?

This has been a topic of research for a long time. In this article, an attempt has been made to study the training parameters of the different networks used. There are other important issues, which needs to be addressed in the future. For example, the possibility of using a more versatile RBNN in terms of a variable design parameter, unlike the present situation where the variable has the same value all around the domain. needs to be addressed. Ways to determine $N N$ 's performance via statistical tools, especially for RBN.V since it employs a linear model to determine the weight associated with each neuron will be investigated.

3) What is the scaling rule between the number of neurons, and computing time. versus number of inputroutput variables and the size of the design data?

There are several rules of thumb for BPNN in the literature (e.g., Greenman [1+]. Carpenter and Barthelemy [4], and Fujita [13]) but to our knowledge, no information is provided for RBNN. 
4) How can one address the need for generating training and testing data most economically and effectively?

The effect of the selection of the design points on accuracy, scaling and performance of polynomial-based RSM has been addressed. The same has yet to be done for NN.

\section{ACKNOWLEDGEMENT}

The present work is partially funded by NASA Marshall Space Flight Center through the Center Director's Discretionary Fund and the Advanced Space Transportation Program/Reusable Launch Vehicle Focused Technology Project.

\section{APPENDIX}

\section{TRAINING DATA}

Table A1. Training Data Sets for 3-D wing model

\begin{tabular}{|c|c|c|c|c|c|c|c|c|}
\hline \multicolumn{3}{|c|}{$\begin{array}{c}\text { Training Data Set \#1 } \\
\text { (9-Simulation) }\end{array}$} & \multicolumn{3}{|c|}{$\begin{array}{l}\text { Training Data Set \# } 2 \\
\text { (15-Simulation) }\end{array}$} & \multicolumn{3}{|c|}{$\begin{array}{l}\text { Training Data Set \# } 3 \\
\text { (25-Simulation) }\end{array}$} \\
\hline AR & $y_{c}$ & $\mathrm{C}_{\mathrm{L}}^{3 / 2} / \mathrm{C}_{\mathrm{D}}$ & AR & $y_{c}$ & $\mathrm{C}_{\mathrm{L}}^{3 / 2} / \mathrm{C}_{\mathrm{D}}$ & AR & $y_{c}$ & $\mathrm{C}_{\mathrm{L}}^{3 / 2} / \mathrm{C}_{\mathrm{D}}$ \\
\hline 1 & 0 & 2.0011 & 1 & 0 & 2.0011 & 1 & 0.0 & 2.0011 \\
\hline 1 & 0.05 & 4.1224 & 1 & 0.05 & 4.1224 & 1 & 0.025 & 4 \\
\hline 1 & 0.1 & 3.6865 & 1 & 0.1 & 3.6866 & 1 & 0.05 & 4.1224 \\
\hline 3 & 0 & 5.6398 & 2 & 0 & 4.03 & 1 & 0.075 & 3.99 \\
\hline 3 & .05 & 9.6873 & 2 & .05 & 7.12 & 1 & 0.1 & 3.6866 \\
\hline 3 & 0.1 & 8.6806 & 2 & 0.1 & 6.34 & 2 & 0.0 & 4.03 \\
\hline 5 & 0 & 7.9413 & 3 & 0 & 5.6398 & 2 & 0.025 & 7.07 \\
\hline 5 & 0.05 & 14.0942 & 3 & 0.05 & 9.6873 & 2 & 0.05 & 7.12 \\
\hline 5 & 0.1 & 12.8951 & 3 & 0.1 & 8.6806 & 2 & 0.075 & 6.89 \\
\hline & & & 4 & 0 & 6.92 & 2 & 0.1 & 6.34 \\
\hline & & & 4 & .05 & 11.99 & 3 & 0.0 & 5.6398 \\
\hline & & & 4 & 0.1 & 10.87 & 3 & 0.025 & 9.64 \\
\hline & & & 5 & 0 & 7.9414 & 3 & 0.05 & 9.6873 \\
\hline & & & 5 & 0.05 & 14.0942 & 3 & 0.075 & 9.39 \\
\hline & & & 5 & 0.1 & 12.8951 & 3 & 0.1 & 8.6806 \\
\hline & & & & & & 4 & 0.0 & 6.92 \\
\hline & & & & & & 4 & 0.025 & 11.86 \\
\hline & & & & & & 4 & 0.05 & 11.99 \\
\hline & & & & & & 4 & 0.075 & 11.66 \\
\hline & & & & & & 4 & 0.1 & 10.87 \\
\hline & & & & & & 5 & 0.0 & 7.9414 \\
\hline & & & & & & 5 & 0.025 & 13.83 \\
\hline & & & & & & 5 & 0.05 & 14.0942 \\
\hline & & & & & & 5 & 0.075 & 13.73 \\
\hline
\end{tabular}


Table A2. Test Data Sets for 3-D wing model based on $A R$ and $y_{c}$

\begin{tabular}{|c|c|c|c|c|c|c|c|c|c|}
\hline \multicolumn{2}{|c|}{ Test Set\#1 for $\mathbf{y}_{\mathbf{c}}$} & \multicolumn{2}{c|}{ Test Set\#2 for $\mathbf{y}_{\mathbf{c}}$} & \multicolumn{2}{c|}{ Test Set\#3 for $\mathbf{y}_{\mathbf{c}}$} & \multicolumn{2}{c|}{ Test Set\#1 for AR } & \multicolumn{2}{c|}{ Test Set\#2 for AR } \\
\hline $\mathrm{AR}$ & $\mathbf{y}_{\mathbf{c}}$ & $\mathrm{AR}$ & $\mathbf{y}_{\mathbf{c}}$ & $\mathrm{AR}$ & $\mathbf{y}_{\mathbf{c}}$ & $\mathbf{A R}$ & $\mathbf{y}_{\mathbf{c}}$ & $\mathrm{AR}$ & $\mathbf{y}_{\mathbf{c}}$ \\
\hline 1 & 0.025 & $\mathbf{1}$ & 0.025 & 1 & 0.0125 & 2 & 0 & 2.5 & 0 \\
\hline 1 & 0.075 & 1 & 0.075 & 1 & 0.0875 & 2 & 0.025 & 2.5 & 0.025 \\
\hline 3 & 0.025 & 2 & 0.025 & 2 & 0.0125 & 2 & 0.05 & 2.5 & 0.05 \\
\hline 3 & 0.075 & 2 & 0.075 & 2 & 0.0875 & 2 & 0.075 & 2.5 & 0.075 \\
\hline 5 & 0.025 & 3 & 0.025 & 3 & 0.0125 & 2 & 0.1 & 2.5 & 0.1 \\
\hline 5 & 0.075 & 3 & 0.075 & 3 & 0.0875 & 4 & 0 & 4.5 & 0 \\
\hline & 4 & 0.025 & 4 & 0.0125 & 4 & 0.025 & 4.5 & 0.025 \\
\hline & 4 & 0.075 & 4 & 0.0875 & 4 & 0.05 & 4.5 & 0.05 \\
\hline
\end{tabular}

Table A3. Performance and heat flux responses for $O / F=4$ for the shear co-axial injector element. (Table

A3-5 together contain 45 data points used as the training set)

\begin{tabular}{|c|c|c|c|c|}
\hline$O / F$ & $V / V_{o}$ & $L_{c o m b}$, in. & $E R E, \%$ & $Q$, Btu/in ${ }^{2}-\mathrm{sec}$ \\
\hline 4.0 & 4.0 & 4.0 & 92.9 & 0.753 \\
\hline 4.0 & 4.0 & 5.0 & 96.0 & 0.753 \\
\hline 4.0 & 4.0 & 6.0 & 97.6 & 0.753 \\
\hline 4.0 & 4.0 & 7.0 & 98.6 & 0.753 \\
\hline 4.0 & 4.0 & 8.0 & 99.0 & 0.753 \\
\hline 4.0 & 6.0 & 4.0 & 95.0 & 0.928 \\
\hline 4.0 & 6.0 & 5.0 & 97.1 & 0.928 \\
\hline 4.0 & 6.0 & 6.0 & 98.5 & 0.928 \\
\hline 4.0 & 6.0 & 7.0 & 99.2 & 0.928 \\
\hline 4.0 & 6.0 & 8.0 & 99.4 & 0.928 \\
\hline 4.0 & 8.0 & 4.0 & 96.6 & 1.10 \\
\hline 4.0 & 8.0 & 5.0 & 98.2 & 1.10 \\
\hline 4.0 & 8.0 & 6.0 & 99.1 & 1.10 \\
\hline 4.0 & 8.0 & 7.0 & 99.4 & 1.10 \\
\hline 4.0 & 8.0 & 8.0 & 99.6 & 1.10 \\
\hline
\end{tabular}


Table A4. Performance and heat flux responses for $O / F=6$ for the shear co-axial injector element.

\begin{tabular}{|c|c|c|c|c|}
\hline$O / F$ & $V / V_{o}$ & $L_{c o m b}$, in. & $E R E, \%$ & $Q$, Btw/in ${ }^{2}$-sec \\
\hline 6.0 & 4.0 & 4.0 & 92.9 & 0.691 \\
\hline 6.0 & 4.0 & 5.0 & 96.0 & 0.691 \\
\hline 6.0 & 4.0 & 6.0 & 97.6 & 0.691 \\
\hline 6.0 & 4.0 & 7.0 & 98.6 & 0.691 \\
\hline 6.0 & 4.0 & 8.0 & 99.0 & 0.691 \\
\hline 6.0 & 6.0 & 4.0 & 95.0 & 0.642 \\
\hline 6.0 & 6.0 & 5.0 & 97.1 & 0.642 \\
\hline 6.0 & 6.0 & 6.0 & 98.5 & 0.642 \\
\hline 6.0 & 6.0 & 7.0 & 99.2 & 0.642 \\
\hline 6.0 & 6.0 & 8.0 & 99.4 & 0.642 \\
\hline 6.0 & 8.0 & 4.0 & 96.6 & 0.741 \\
\hline 6.0 & 8.0 & 5.0 & 98.2 & 0.741 \\
\hline 6.0 & 8.0 & 6.0 & 99.1 & 0.741 \\
\hline 6.0 & 8.0 & 7.0 & 99.4 & 0.741 \\
\hline 6.0 & 8.0 & 8.0 & 99.6 & 0.741 \\
\hline
\end{tabular}

Table A5. Performance and heat tlux responses for $O / F=8$ for the shear co-axial injector element.

\begin{tabular}{|c|c|c|c|c|}
\hline $\boldsymbol{O} / \boldsymbol{F}$ & $\boldsymbol{V} / \boldsymbol{V}_{o}$ & $\boldsymbol{L}_{\text {comb }}$, in. & $E R E, \boldsymbol{c}$, & $Q, \mathrm{Btu} / \mathbf{i n}^{2}$-sec \\
\hline 8.0 & 4.0 & 4.0 & 92.9 & 0.588 \\
\hline 8.0 & 4.0 & 5.0 & 96.0 & 0.588 \\
\hline 8.0 & 4.0 & 6.0 & 97.6 & 0.588 \\
\hline 8.0 & 4.0 & 7.0 & 98.6 & 0.588 \\
\hline 8.0 & 4.0 & 8.0 & 99.0 & 0.588 \\
\hline 8.0 & 6.0 & 4.0 & 95.0 & 0.512 \\
\hline 8.0 & 6.0 & 5.0 & 97.1 & 0.512 \\
\hline 8.0 & 6.0 & 6.0 & 98.5 & 0.512 \\
\hline 8.0 & 6.0 & 7.0 & 99.2 & 0.512 \\
\hline 8.0 & 6.0 & 8.0 & 99.4 & 0.512 \\
\hline 8.0 & 8.0 & 4.0 & 96.6 & 0.493 \\
\hline 8.0 & 8.0 & 5.0 & 98.2 & 0.493 \\
\hline 8.0 & 8.0 & 6.0 & 99.1 & 0.493 \\
\hline 8.0 & 8.0 & 7.0 & 99.4 & 0.493 \\
\hline 8.0 & 8.0 & 8.0 & 99.6 & 0.493 \\
\hline
\end{tabular}


Table A6. Data used to test the polynomials and NN for the shear co-axial injector element. (The table contains 20 data points used as the testing set)

\begin{tabular}{|c|c|c|c|c|}
\hline$O / F$ & $V / V_{0}$ & $L_{\text {comb }}$ in. & $E R E, \%$ & $Q$, Btu/in ${ }^{2}$-sec \\
\hline 4.0 & 5.0 & 4.0 & 94.4 & 0.812 \\
\hline 4.0 & 5.0 & 5.0 & 96.9 & 0.812 \\
\hline 4.0 & 5.0 & 6.0 & 98.1 & 0.812 \\
\hline 4.0 & 5.0 & 7.0 & 99.1 & 0.812 \\
\hline 4.0 & 5.0 & 8.0 & 99.4 & 0.812 \\
\hline 4.0 & 7.0 & 4.0 & 96.0 & 1.014 \\
\hline 4.0 & 7.0 & 5.0 & 97.9 & 1.014 \\
\hline 4.0 & 7.0 & 6.0 & 98.8 & 1.014 \\
\hline 4.0 & 7.0 & 7.0 & 99.4 & 1.014 \\
\hline 4.0 & 7.0 & 8.0 & 99.6 & 1.014 \\
\hline 6.0 & 5.0 & 4.0 & 94.4 & 0.642 \\
\hline 6.0 & 5.0 & 5.0 & 96.9 & 0.642 \\
\hline 6.0 & 5.0 & 6.0 & 98.1 & 0.642 \\
\hline 6.0 & 5.0 & 7.0 & 99.1 & 0.642 \\
\hline 6.0 & 5.0 & 8.0 & 99.4 & 0.642 \\
\hline 6.0 & 7.0 & 4.0 & 96.0 & 0.691 \\
\hline 6.0 & 7.0 & 5.0 & 97.9 & 0.691 \\
\hline 6.0 & 7.0 & 6.0 & 98.8 & 0.691 \\
\hline 6.0 & 7.0 & 7.0 & 99.4 & 0.691 \\
\hline 6.0 & 7.0 & 8.0 & 99.6 & 0.691 \\
\hline
\end{tabular}

Table A7. Propellant momentum ratio as a function of propellant pressure drops: shear co-axial injector element.

\begin{tabular}{|l|l|l|l|l|l|l|l|}
\hline & \multicolumn{7}{|c|}{$\Delta \boldsymbol{P}_{o}$} \\
\hline$\Delta \boldsymbol{P}_{f}$ & $\mathbf{2 0 0}$ & $\mathbf{1 8 0}$ & $\mathbf{1 6 0}$ & $\mathbf{1 5 0}$ & $\mathbf{1 4 0}$ & $\mathbf{1 2 0}$ & $\mathbf{1 0 0}$ \\
\hline $\mathbf{2 0 0}$ & 1.49 & 1.42 & 1.33 & $\mathbf{1 . 3 0}$ & 1.25 & 1.16 & $\mathbf{1 . 0 6}$ \\
\hline $\mathbf{1 8 0}$ & 1.57 & 1.50 & 1.41 & 1.37 & 1.32 & 1.22 & 1.11 \\
\hline $\mathbf{1 6 0}$ & 1.67 & 1.59 & 1.50 & 1.45 & 1.40 & 1.30 & 1.18 \\
\hline $\mathbf{1 5 0}$ & 1.73 & 1.64 & 1.54 & $\mathbf{1 . 4 9}$ & 1.44 & 1.34 & 1.22 \\
\hline $\mathbf{1 4 0}$ & 1.79 & 1.70 & 1.60 & 1.55 & 1.50 & 1.39 & 1.27 \\
\hline $\mathbf{1 2 0}$ & 1.93 & 1.83 & 1.72 & 1.67 & 1.61 & 1.50 & 1.37 \\
\hline $\mathbf{1 0 0}$ & $\mathbf{2 . 1 1}$ & 2.00 & 1.89 & $\mathbf{1 . 8 3}$ & 1.77 & 1.64 & $\mathbf{1 . 4 9}$ \\
\hline
\end{tabular}


Table A8. Design data for a shear co-axial injector element with $\Delta P_{o}$ and $\Delta P_{i}=200 \mathrm{psi}$

\begin{tabular}{|l|l|l|l|l|l|l|l|l|}
\hline$\Delta \mathbf{P}_{\mathbf{Q}}$ & $\mathbf{A P}_{\mathbf{r}}$ & $\mathbf{L}_{\text {comb }}$ & $\alpha$ & $\mathbf{E R E}$ & $\mathbf{Q}_{\mathbf{w}}$ & $\mathbf{H}_{\text {impinge }}$ & $\mathbf{W}_{\text {rel }}$ & $\mathbf{C}_{\text {rel }}$ \\
\hline 200 & 200 & 2 & 15 & $\mathbf{V A}$ & 0.85 & 0.84 & 0.923 & 1.083 \\
\hline 200 & 200 & 2 & 20 & 85 & 0.85 & 0.62 & 0.923 & 1.083 \\
\hline 200 & 200 & 2 & 30 & 92.8 & 0.85 & 0.39 & 0.923 & 1.083 \\
\hline 200 & 200 & 2 & 45 & 95.4 & 0.85 & 0.23 & 0.923 & 1.083 \\
\hline 200 & 200 & 2 & 50 & 95.8 & 0.85 & 0.19 & 0.923 & 1.083 \\
\hline & & & & & & & & \\
\hline 200 & 200 & 4 & 15 & 91 & 0.85 & 0.84 & 1 & 1.083 \\
\hline 200 & 200 & 4 & 20 & 95.2 & 0.85 & 0.62 & 1 & 1.083 \\
\hline 200 & 200 & 4 & 30 & 96.8 & 0.85 & 0.39 & 1 & 1.083 \\
\hline 200 & 200 & 4 & 45 & 98.1 & 0.85 & 0.23 & 1 & 1.083 \\
\hline 200 & 200 & 4 & 50 & 98.4 & 0.85 & 0.19 & 1 & 1.083 \\
\hline & & & & & & & & \\
\hline 200 & 200 & 6 & 15 & 95.6 & 0.85 & 0.84 & 1.077 & 1.083 \\
\hline 200 & 200 & 6 & 20 & 97.8 & 0.85 & 0.62 & 1.077 & 1.083 \\
\hline 200 & 200 & 6 & 30 & 98.5 & 0.85 & 0.39 & 1.077 & 1.083 \\
\hline 200 & 200 & 6 & 45 & 99.2 & 0.85 & 0.23 & 1.077 & 1.083 \\
\hline 200 & 200 & 6 & 50 & 99.4 & 0.85 & 0.19 & 1.077 & 1.083 \\
\hline & & & & & & & & \\
\hline 200 & 200 & 8 & 15 & 98.3 & 0.85 & 0.84 & 1.154 & 1.083 \\
\hline 200 & 200 & 8 & 20 & 99.1 & 0.85 & 0.62 & 1.154 & 1.083 \\
\hline 200 & 200 & 8 & 30 & 99.4 & 0.85 & 0.39 & 1.154 & 1.083 \\
\hline 200 & 200 & 8 & 45 & 99.6 & 0.85 & 0.23 & 1.154 & 1.083 \\
\hline 200 & 200 & 8 & 50 & 99.7 & 0.85 & 0.19 & 1.154 & 1.083 \\
\hline
\end{tabular}




\section{REFERENCES}

[1] Baysal, O. and Eleshaky, M.E., 1992 "Aerodynamic Design Optimization Using Sensitivity Analysis and CFD", AIAA Journal Vol.30 pp.718-725.

[2] Bauer Jr., K. W., Parnell, G. S., and Myers, D. A., "Response Surface Methodology as a Sensitivity Analysis Tool in Decision Analysis," Journal of Multi-Criteria Decision Analysis, Vol. 8, pp.162-180. 1999.

[3] Calhoon, D., Ito, J. and Kors, D., "Investigation of Gaseous propellant Combustion and Associated Injector-Chamber Design Guidelines," Aerojet liquid rocket company, NASA Cr-121234. Contract NAS3-13379, 1973.

[4] Carpenter, W.C. and Barthelemy, J.-F.M, "A comparison of Polynomial Approximations and Artificial Neural Nets as Response Surface," Structural Optimization, Vol. 5. pp. 166-174, 1993.

[5] Coleman, T.. Branch, M. A. and Grace, A., Optimization Toolbox for use with Matlab, Version 2. The Math Works Inc, 1999.

[6] Dadone, A., Valorani, M. and Grossman, B., "Smoothed Sensitivity Equation Method for Fluid Dynamic Design Problems." AlAA Journal, Vol. 38, pp. 418-426, 2000.

[7] Dean, E. B., "Taguchi Methods from the Perspective of Competitive Advantage," http://akao. Larc.nasa.gov/dfc/tm.html, 1996.

[8] Dernuth, H., and Beale M.. Matlab Neural Network Toolbox, The Math Works Inc, 1992.

[9] Drakos, N., Mathematical Optimization, Electronic Book of Computer Based Learning Unit, University of Leeds (http://csepl phy ornl gov/mo/mo.html), 1994.

[10] Elbanna, H.M. and Carlson, L.A., "Aerodynamic Sensitivity Coefficients Using the ThreeDimensional Full Potential Equations", J. Aircraft Vol.31, pp.1071-1077, 1994.

[11] Faller, W.E. and Schereck, S. J, "Unsteady Fluid Mechanics Applications of Neural Networks." 33rd AIAA Aerospace Sciences Meeting and Exhibit, Reno, Nevada, AIAA 95-0529, 1995.

[12] Fan, X., Herbert, T., and Haritonidis, J.H., "Transition Control with Neural Networks," 33rd AIAA Aerospace Sciences Meeting and Exhibit, Reno, Nevada, AIAA 95-0674, 1995.

[13] Fujita, O. "Statistical estimation of the number of hidden units for feedforward neural networks," : Veural Networks, 11, pp. 851-859, 1998. 
[14] Greenman, R. M., "2-D High-Lift Aerodynamic Optimization L'sing Neural Networks." NASA TM-1998-112233, June 1998, pp. 146, 1998.

[15] Greenman, R. M. and Roth, K.R., "High-Lift Optimization Design Using Neural Network," Proceedings of DETC98, ASME Computers in Engineering Conference, Atlanta, Georgia, DETC98/CIE$6006,1998$.

[16] Greenman, R. M. and Roth, K.R., "Minimizing Computational Data Requirements For MultiElement Airfoils Using Neural Networks," 37th AIAA Aerospace Sciences Meeting and Exhibit, Reno, Nevada, AIAA 99-0258, 1999.

[17] Giunta, A. A., Dudley, J. M., Narducci, R., Grossman, B., Haftka, R. T., Mason, W. H., and Watson, L. T., "Noisy Aerodynamic Response and Smooth Approximations in HSCT Design," AIAA Paper 94-4376, 1994

[18] Haftka, R.T. and Gurdal, Z., Elements of Structural Optimization, Kluwer Academic Publishers, 1991.

[19] Haftka, R.T., Scott, E.P., and Cruz, J.R., "Optimization and Experiments: A Survey," Applied Mechanics Reviews, 51(7), pp. 435-448, 1998.

[20] Huang, S.Y., Miller, L. S., and Steck, J. E., "An Exploratory Application of Neural Networks to Airfoil Design," 34th AIAA Aerospace Sciences Meeting and Exhibit, Reno. Nevada, AIAA 94-0501. 1994

[21] Illi, O. J., Greitzer, F. L., Kangas, L. J., and Reeve, T. J., "An Artificial Neural Network System for Diagnosing Gas Turbine Engine Fuel Faults," 48th Meeting of the Mechanical Failures Prevention Group (MFPG48), 1994

[22] Jang, J.-S. R., Sun, C.-T., and Mizutani, E., Neuro-Fuzzy and Soft Computing: A Computational Approach To Learning and Machine Intelligence, Prentice Hall, 1997.

[23] JMP version 3, Statistics And Graphics Guide, SAS Institute Inc., 1998.

[24] Kangas, L. J., Greitzer, F. L., and Illi, O. J., "TEDANN: Turbine Engine Diagnostic Artificial Neural Network," The American Defense Preparedness Association (ADPA) Viational Symposium and Exhibition on Advanced Information Systems and Technology, 1994. 
[25] Kosko, B, Neural Nerworks and Fuzzy Systems: A Dynamical Systems Approach to Machine Intelligence, Prentice Hall, 1992.

[26] Ladson, L.S., Waren, A. D., Jain, A., and Ratner, M.. "Design and Testing of a Generalized Reduced Gradient Code for Non-linear Programming," ACM Transactions on Mathematical Software. Vol. 4, No. 1, pp.51-56, 1978

[27] Lambert, P.A., Lecordix, J.L., and Braibant, V., "Constrained Optimization of Nacelle Shapes in Euler Flow using Semi-analytical Sensitivity Analysis" Structural Optimization, Vol.10, pp.239-246, 1995.

[28] Lavretsky, E., "Ordered Neural Networks: Formulation, Analysis, Design and Application," 37th AIAA Aerospace Sciences Meeting and Exhibit, Reno, Nevada, AIAA 99-0106, 1999.

[29] Laurence, S., Giles, C. L., and Tsoi, A. C. "What size of Neural Network Gives Optimal Generalization? Convergence Properties of Back Propagation," Technical Report, UMIACS-TR-96-22 and CS-TR-3617, Institute for Advanced Computer Studies, University of Maryland, 1996.

[30] Lawrence, S., Tsoi, A. C, and Giles, C. L., "Correctness, Efficiency, Extendibility and Maintainability in Neural Network Simulation," International Conference on Neural Networks, ICNN 96, June 2-6, Washington DC. IEEE Press, pp. 474-479, 1996.

[31] Madavan, N.K. Rai, M.M. and Huber, F. W.. "Neural Net-Based Redesign of Transonic Turbines for Improved L'nsteady Aerodynamic Performance," AIAA/SAE/ASME/ASEE 35th Joint Propulsion Conference, AlAA 99-2522, June 20-24., 1999.

[32] Madsen, J.I., "Design Optimization of Internal Flow Devices," Philosophy of Doctorate Thesis, Aalborg Lniversity, 1998.

[33] Madsen, J.I., Shyy, W. and Haftka, R.T, "Response Surface Techniques for Diffuser Shape Optimization," accepted for publication in AIAA Journal, 1999

[34] Maghami, P. G., and Sparks Jr., D.W. "Design of Neural Networks for fast Convergence and Accuracy," 39th AIAA/ASME/ ASCEAHS/ASC Structures, Structural Dynamics, and Materials Conference, Long Beach. California. AIAA 98-1780, 1998. 
[35] Maghami, P. G., and Sparks Jr., D.W, "Design of Neural Networks for Fast Convergence and Accuracy: Dynamics and Control," IEEE Transactions on Neural networks, vol. 11, no.1, pp. 113-123, 2000 .

[36] Microsoft Excel 97, Microsoft Corporation (1985-1996).

[37] Myers, R. H., and Montgomery, D. C., Response Surface Methodology - Process and Product Optimization Using Designed Experiments, New York: John Wiley \& Sons, Inc., 1995.

[38] Nash, S. G., and Sofer, A., Linear and Nonlinear Programming , McGraw Hill Inc., 1984.

[39] Nikolaidis, E., Long, L., and Ling, Q., "Neural Networks and Response Surface Polynomials for Design of Vehicle Joints," 39th AIAA/ASME/ ASCEJAHS/ASC Structures, Structural Dynamics, and Materials Conference, Long Beach, California, AIAA-98-1777, 1998.

[40] Norgaard, M., Jorgenson, C. C., and Ross, J. C., "Neural Network Prediction of New Aircraft Design Coefficients," NASA TM-112197, May 1997, pp. 20, 1997.

[41] Orr, M. J. L., "Introduction to Radial Basis Function Networks," http://www.anc.ed.ac.uk/-mjo/rbf.html, 1996.

[42] Orr, M. J. L., " Optimising the Widths of RBFs," Fifth Brazilian Symposium on Neural Networks, Belo Horizonte, Brazil, 1998.

[43] Orr, M. J. L., "Recent Advances in Radial Basis Function Networks." http://www.anc.ed.ac.uk/-mjo/rbf.html, 1999.

[44] Owen. A., "Orthogonal Arrays for: Computer Experiments, Integration and Visualization." Statistica Sinica, Vol. 2, No.2, pp. 439-452, 1992.

[45] Owen, A., "Orthogonal Arrays for: Computer Experiments, Visualization, and Integration in high dimensions," http://src.doc.ic.ac.uk/public/pub/Mirrors/lib.stat.cmu. edu/designs/owen.readme, 1994.

[46] Owen, A., "Lattice Sampling Revisited: Monte Carlo Variance of Means over Randomized Orthogonal Arrays," The Annals of Statistics, Vol. 4, No. 2, pp.930-945, 1994.

[47] Owen, A.. "Overtitting in Neural Networks," hup://www-stat.stanford.edu/-owen /reports/. 1994. 
[48] Papila, M. and Haftka, R. T., "Uncertainty and Wing Structural Weight Approximations," $40^{\text {th }}$ AIAA/ASME/ASCE/AHS/ASC Structures, Structural Dynamics and Materials Conference, St Louis MO, AIAA-99-1312, 1999.

[49] Papila, N., Shyy, W., Fitz-Coy, N. and Haftka, R.T.. "Assessment of Neural Net and Polynomial-Based Techniques for Aerodynamic Applications, " 17th Applied Aerodynamics Conference, Norfolk, VA, AIAA 99-3167, 1999.

[50] Papila, N., Shyy, W., Griffin, L., Huber,F., and Tran, K., "Preliminary Design Optimization For A Supersonic Turbine For Rocket Propulsion," 36th AIAA/ASME/SAE/ASEE Joint Propulsion Conference and Exhibit, Huntsville, AL, Paper No.2000-3242, 2000.

[51] Rai, M.M. and Madavan, N.K, "Application of Artificial Neural Networks to the Design of Turbomachinery Airfoils," 36th AIAA Aerospace Sciences Meeting and Exhibit, Reno, Nevada, AlAA $2000-0169,1998$.

[52] Rai, M.M. and Madavan, N.K. "Aerodynamic Design Using Neural Networks," 7th AIAA/USAF/ NASA/ISSMO Symposium on Multidisciplinary Analysis and Optimization, St. Louis, AIAA $98-4928,1998$.

[53] Rai, M.M. and Madavan, N.K, "Improving the Unsteady Aerodynamic Performance of Transonic Turbines L'sing Neural Networks." 38th AIAA Aerospace Sciences Meeting and Exhibit, Reno, Nevada, AIAA $2000-0169,2000$.

[54] Reuther, J., Alonso, J.J., Rimlinger, M.J. and Jameson, A., “Acrodynamic Shape Optimization of Supersonic Aircraft Configurations via an Adjoint Formulation on Distributed Memory Parallel Computers," Computers and Fluids, Vol. 28, pp. 675-700, 1999.

[55] Ross, J. C., Jorgenson, C. C., and Norgaard, M., "Reducing Wind Tunnel Data Requirements Using Neural Networks, "NASA TM-112193, May 1997, pp. 15, 1997.

[56] Rumelhart, D. E., McClelland, J. L., and the PDP Group, Parallel Distributed Processing, Vol. I and II. MIT Press, 1986.

[57] Shyy, W., Tucker, P.K. and Vaidyanathan, R., "Response Surface and Neural Network Techniques for Rocket Engine Injector Optimization," AIAA/SAE/ASME/ASEE 35th Joint Propulsion Conference, AIAA 99-2455, June 20-24, 1999. 
[58] Shyy, W., Papila, N., Tucker, P. K., Vaidyanathan, R., and Griffin L., "Global Design Optimization for Fluid Machinery Applications," The Second International Symposium On Fluid Machinery And Fluid Engineering, October, Beijing, China, 2000.

[59] Sobieszczanski-Sobieski, J. and Haftka, R.T., "Multidisciplinary aerospace design optimization: survey of recent developments," Structural Optimization, Vol. 14, pp.1-23, 1997.

[60] Sparks, Jr., D.W. and Maghami, P. G., "Neural Networks for Rapid Modeling and Analysis," 39th AIAA/ASME/ASCE/AHS/ASC Structures, Structural Dynamics, and Materials Conference, Long Beach, California, AIAA 98-1779, 1998.

[61] Stepniewski, S. W., and Jorgenson, C. C., "Toward a More Robust Pruning Procedure for MLP Networks," NASA/TM-1998-112225, April 1998, pp. 16, 1998.

[62] Stepniewski, S. W., Greenman, R. M., Jorgenson, C. C. and Roth, K.R., "Designing Compact Feedforward Neural Models with Small Training Data Sets," 38th AIAA Aerospace Sciences Meeting and Exhibit, Reno, Nevada, AIAA 2000-0170, 2000

[63] Trosset, M. W., and Torczon, V., "Numerical Optimization Using Computer Experiments," NASA CR-201724 ICASE Report No.9738, August 1997.

[64] Tucker, P.K., Shyy, W. and Sloan, J.G., "An Integrated Design/Optimization Methodology for Rocket Engine lnjectors," AIAA/SAE/ASME/ASEE 34th Joint Propulsion Conference, AIAA-98-3513, 1998.

[65] Tucker, P.K., Shyy, W. and Vaidyanathan, R., "An Optimization-Based Approach to Injector Element Design," AIAA/SAE/ASME/ASEE 35th Joint Propulsion Conference, Paper No. 2000-3220. 2000

[66] Unal, R., and Dean, E. B., "Taguchi Approach to Design Optimization for Quality and Cost: An Overview," Proceedings of the International Society of Parametric Analysts $13^{\text {th }}$ Annual Conference. New Orleans, Louisiana, 1991.

[67] Unal, R., and Dean, E. B., "Design For Cost And Quality: The Robust Design Approach," http://mijuno.larc.nasa.gov/pap/robdes/robdes.html, 1995. 
[68] Unal, R., Braun, R. D., Moore, A.A., and Lepsch, R.A., "Design Optimization on Cost Basis Using Taguchi`s Orthogonal Arrays," Proceedings of the Annual American Society for Engineering Management (ASEM) Conference, 1996.

[69] Unal, R., Braun, R. D., Moore, A.A., and Lepsch, R.A., "Response Surface Model Building Using Orthogonal Arrays for Computer Experiments." $19^{\text {th }}$ Annual International Conference of the International Society of Parametric Analysis, New Orleans, Louisiana, 1997.

[70] Unal, R., Lepsch, R. A., and Mc.Millin, M. L., "Response Surtace Model Building and Multidisciplinary Optimization using D-Optimal Designs," $7^{\text {th }}$ AIAA/USAF/NASA/ISSMO Symposium on Multidisciplinary Analysis and Optimization, Paper No. 98-4759, 1998.

[71] Vaidyanathan, R., Papila, N., Shyy, W., Tucker, P.K., Griffin, L. W., Fitz-Coy, N. and Haftka, R.T, "Neural Network-Based and Response Surface-Based Optimization Strategies for Rocket Engine Component Design," 8th AIAA/USAF/AASA/ISSMO Symposium on Multidisciplinary Analysis and Optimization, Long Beach, CA, 2000.

[72] Vanderplaats, G. N., Numerical Optimization Techniques for Engineering Design With Applications, McGraw Hill, Inc., 1984. 
9. FIGURES

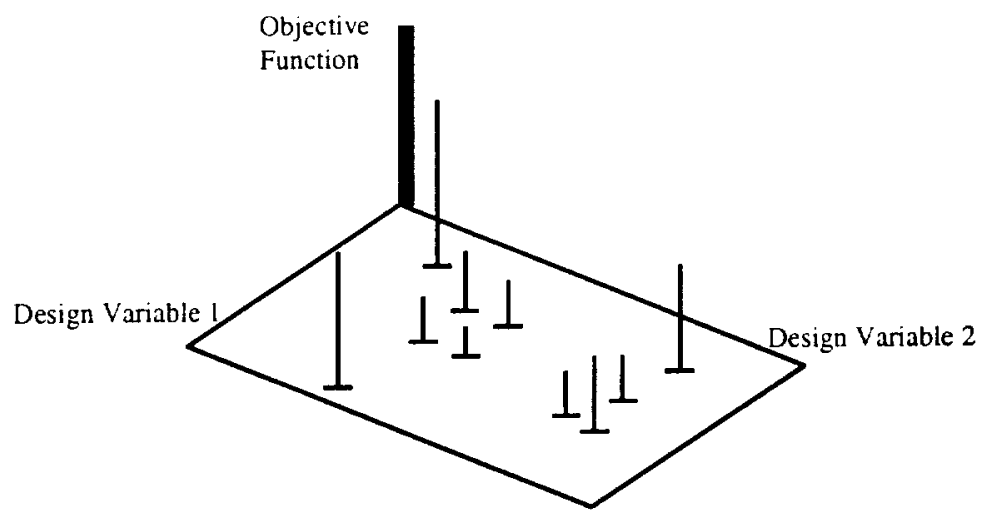

STEP I: Defining and populating the design space

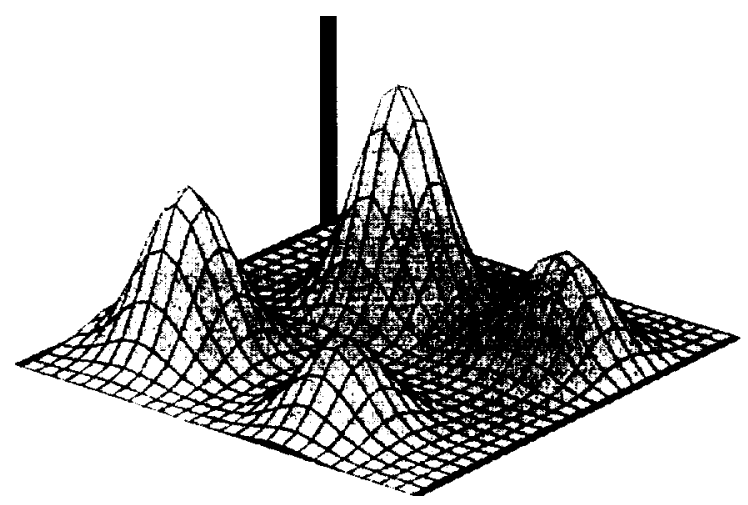

STEP II: Interpolating with response function

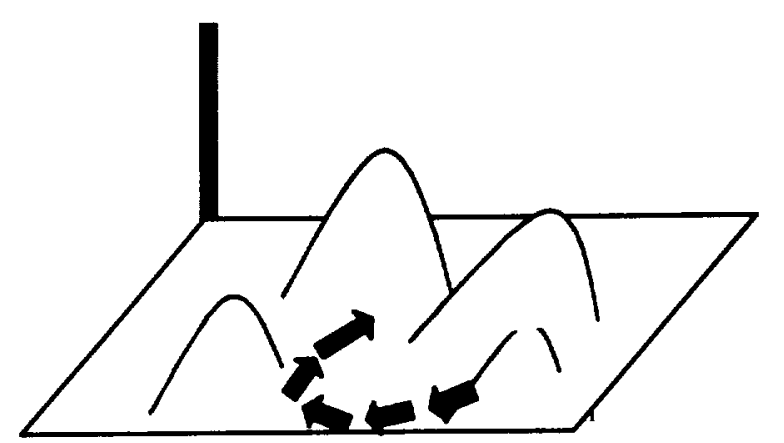

Figure 1. Schematic of the procedure for global design optimization 


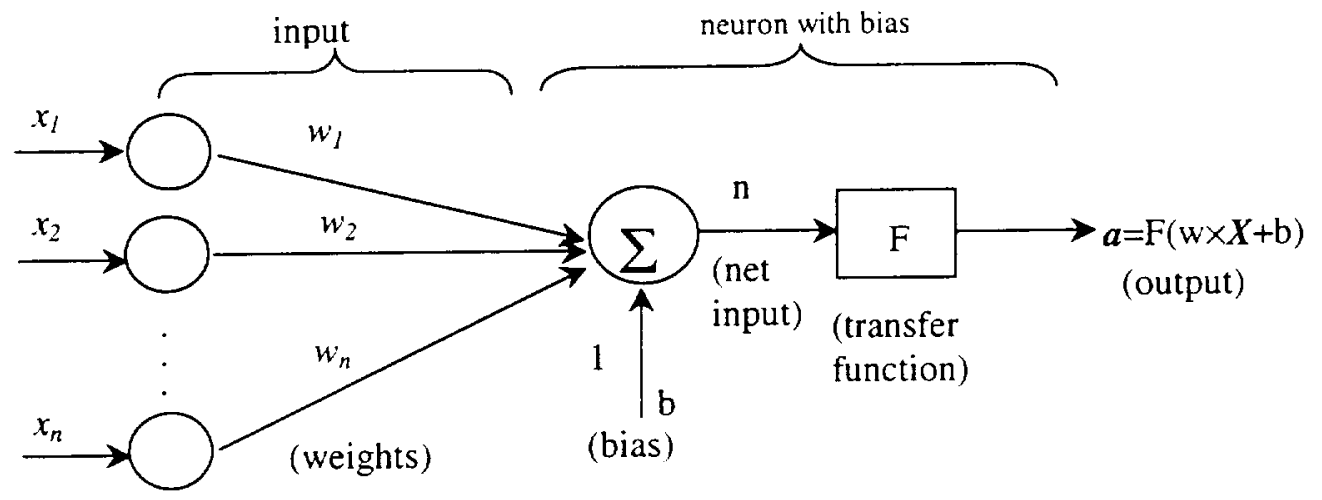

Figure 2. Schematic of a simple neuron model

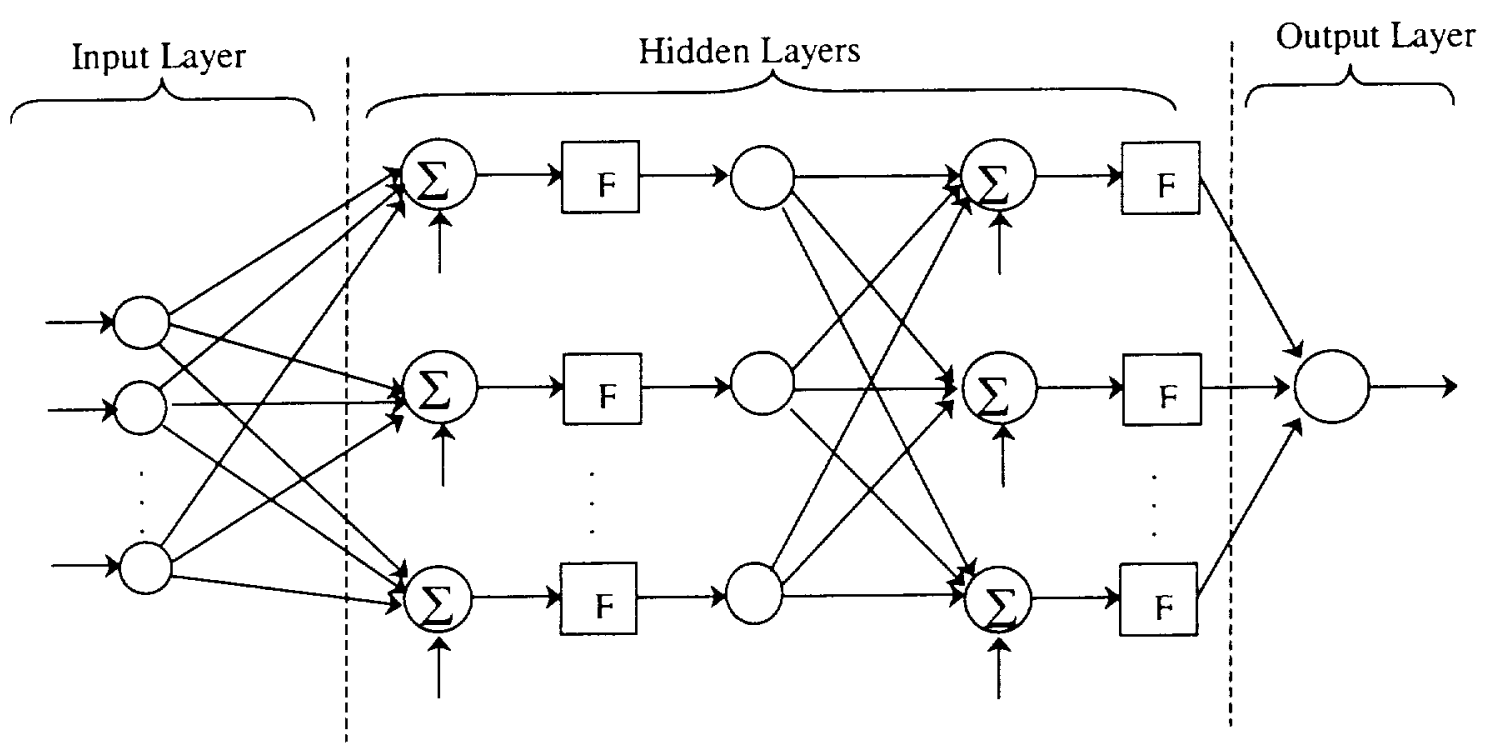

Figure 3. Schematic of a neural network with 2-hidden layers 


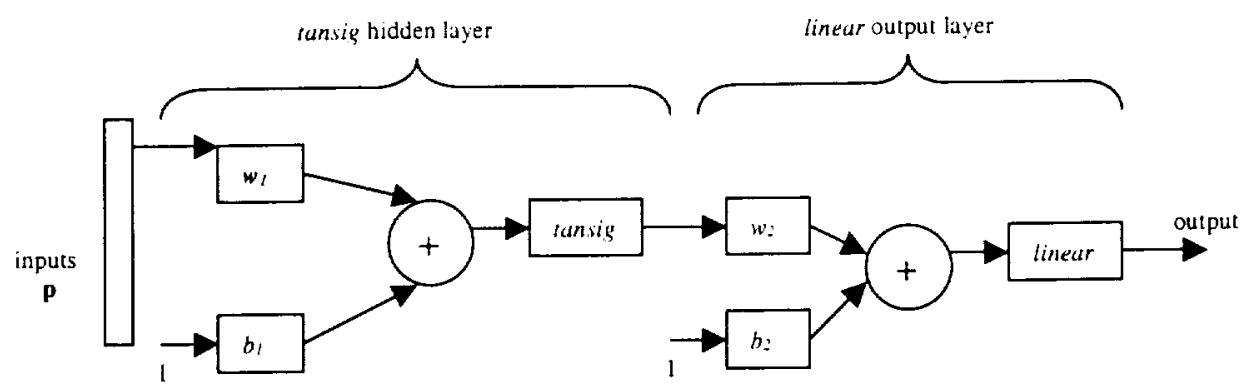

(a)

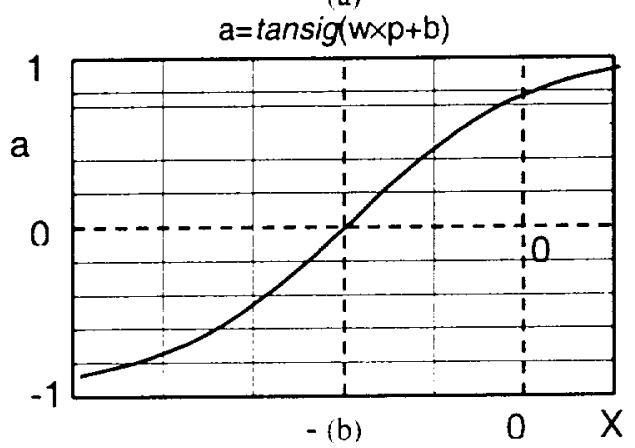

Figure 4. (a) Back-propagation neural network architecture, (b) Back-propagation transfer function, tansig

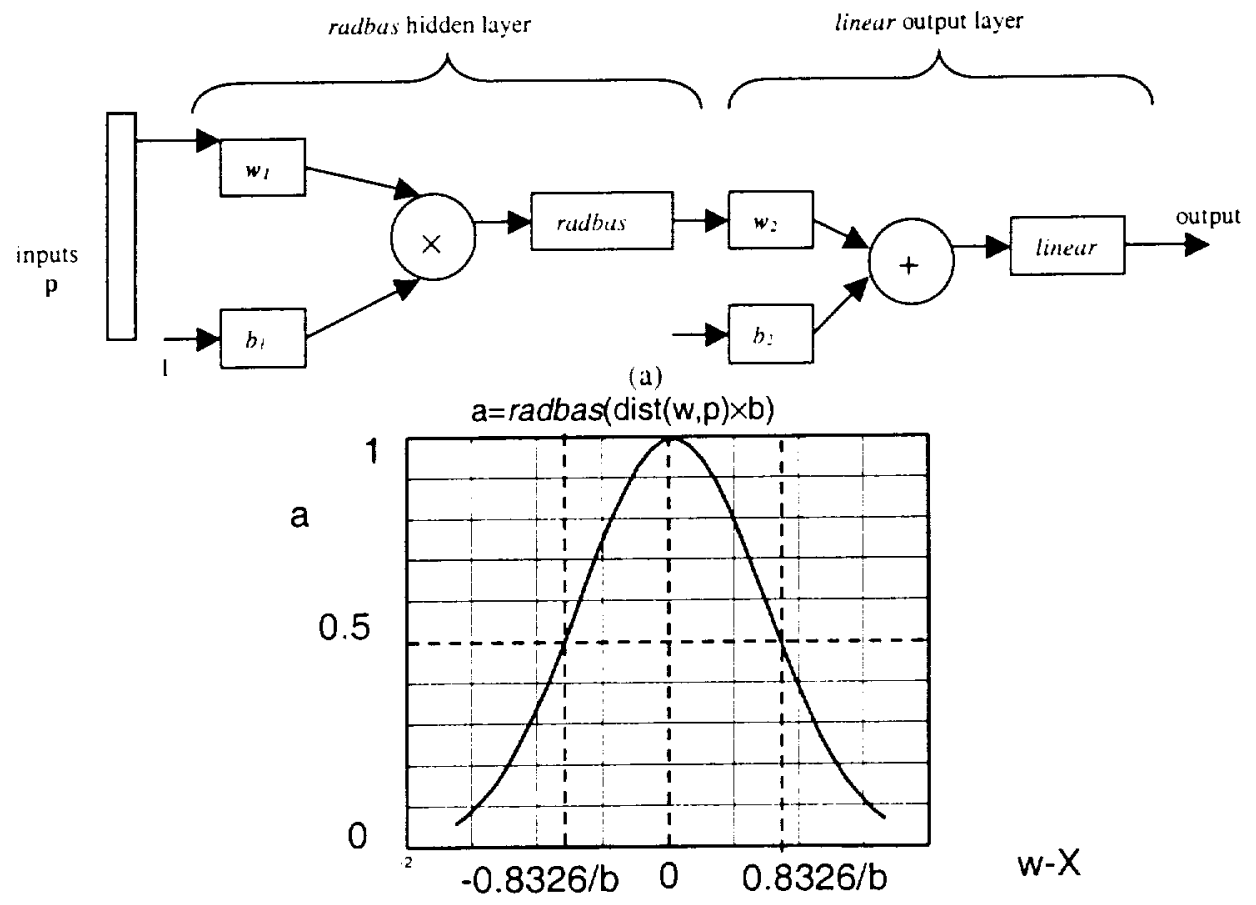

(b)

Figure 5. (a) Radial basis neural network architecture, (b) Radial-basis transter function, radbas. 


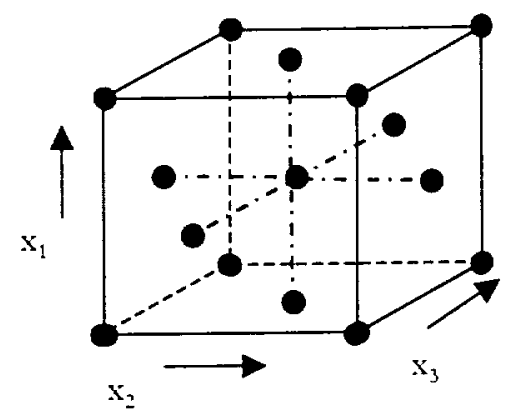

Figure 6. Face centered composite designs (FCCD) for 3 design variables; $x_{1}, x_{2}$, and $x_{3}$

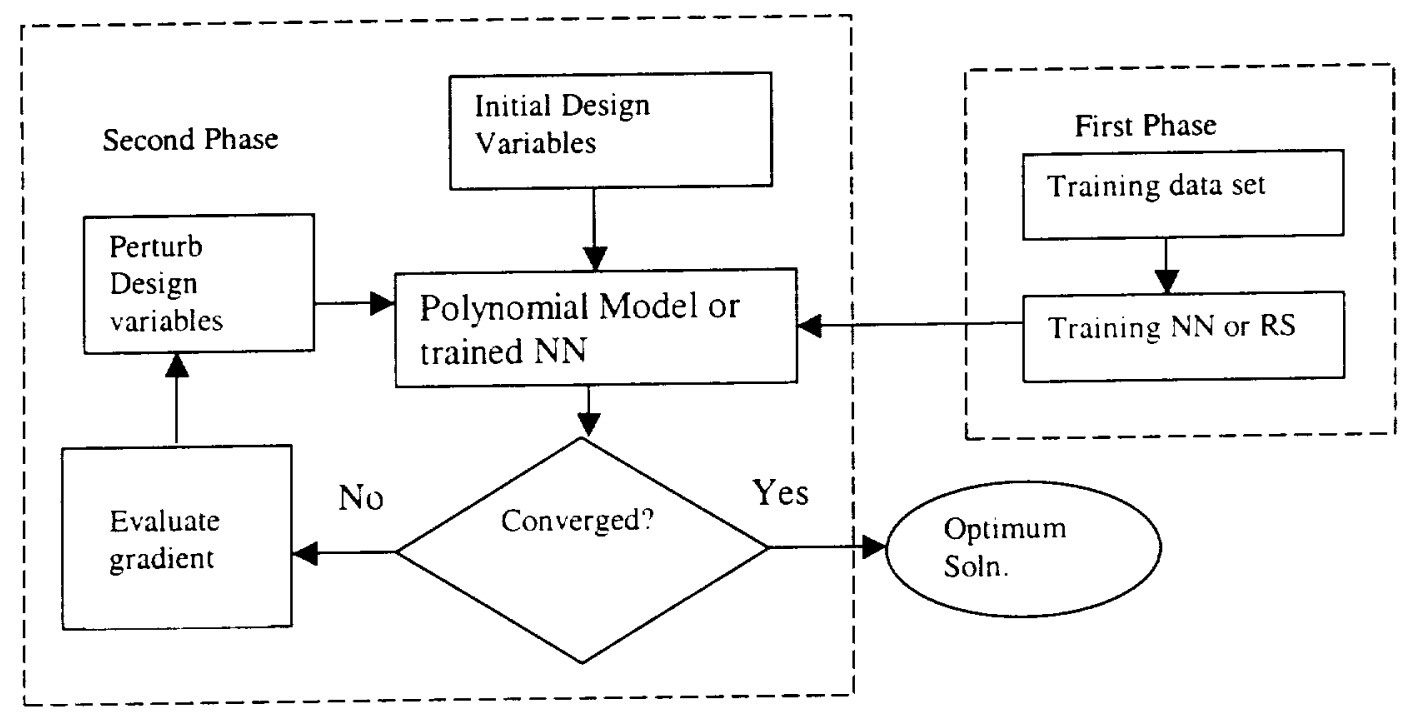

Figure 7. The two phases of the optimization process, where Phase 1 deals with data processing/generating and Phase 2 deals with optimization. 


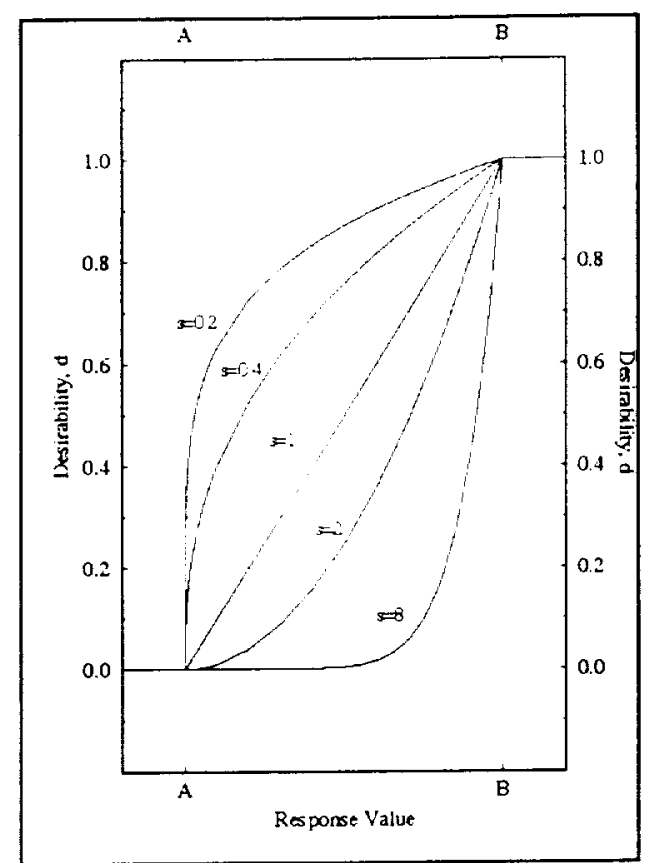

Figure 8. Desirability tunction for various weight factors, $s$.

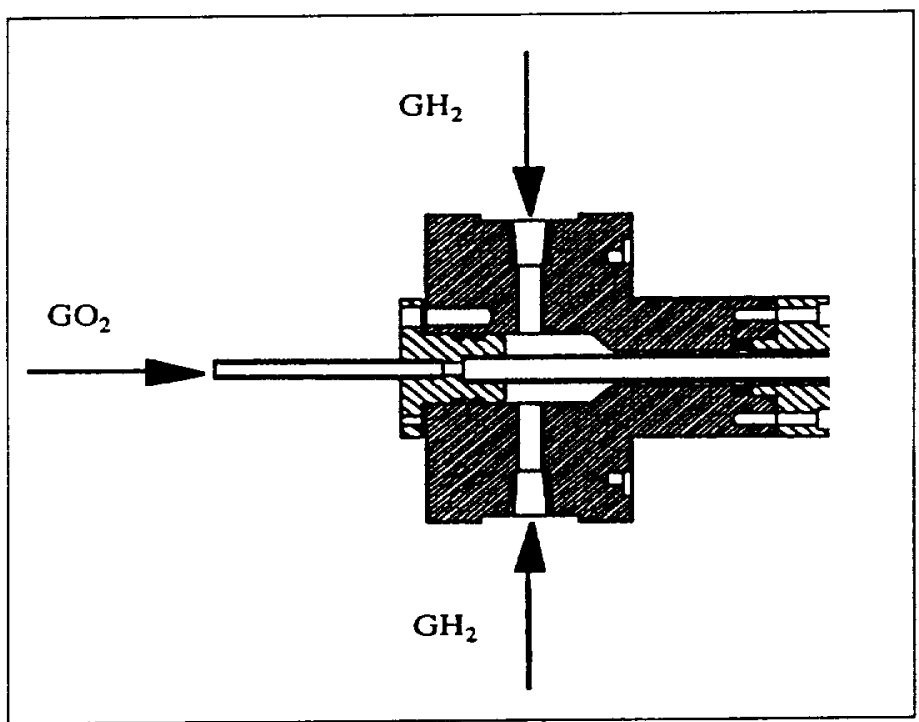

Figure 9. Schematic of shear co-axial injector element 


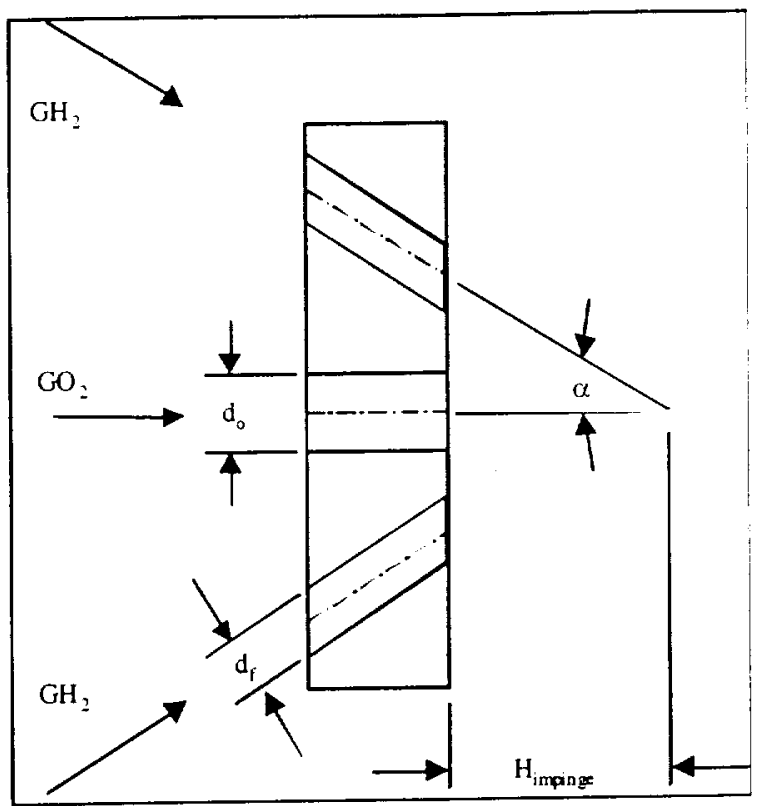

Figure 10. Schematic of impingement injector element

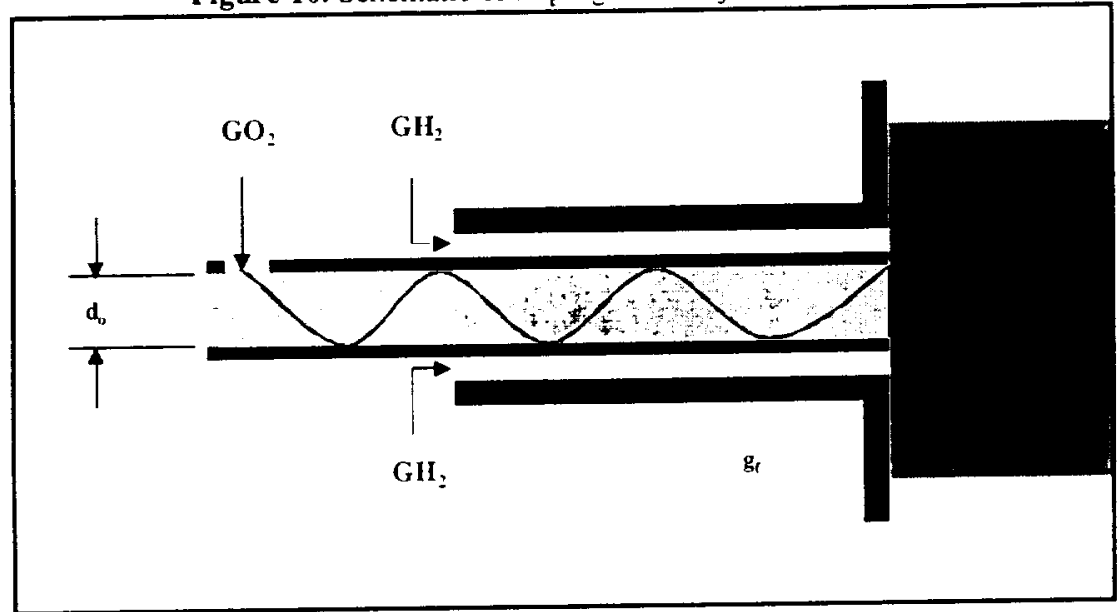

Figure 11. Swirl co-axial injector element schematic

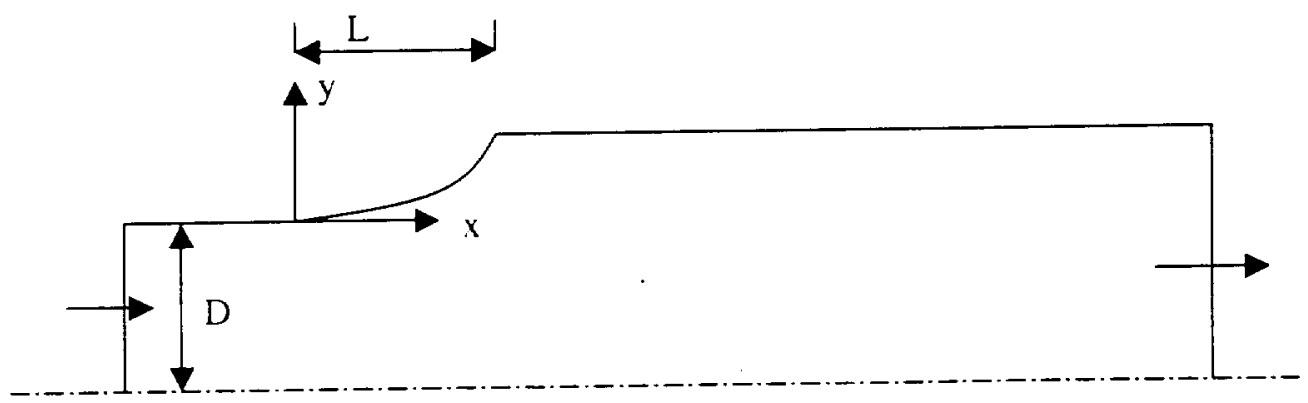

Figure 12. Two-dimensional symmetric diffuser subjected to shape optimization in terms of pressure recovery measured between in- and outlet. 


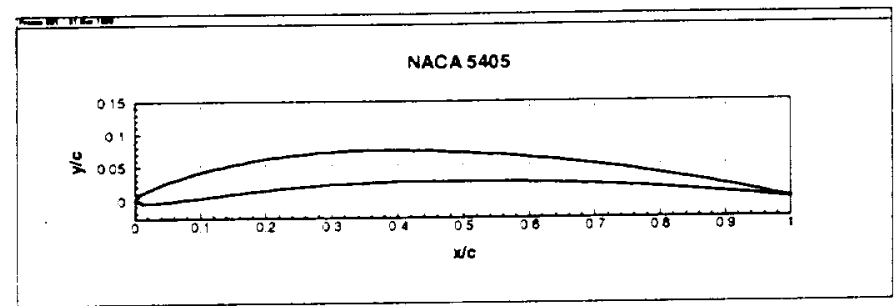

Figure 13. NACA 5405 profile

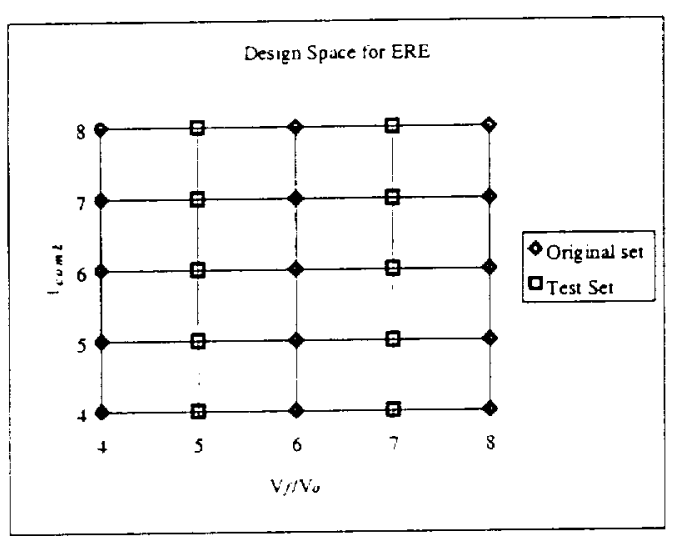

(a)

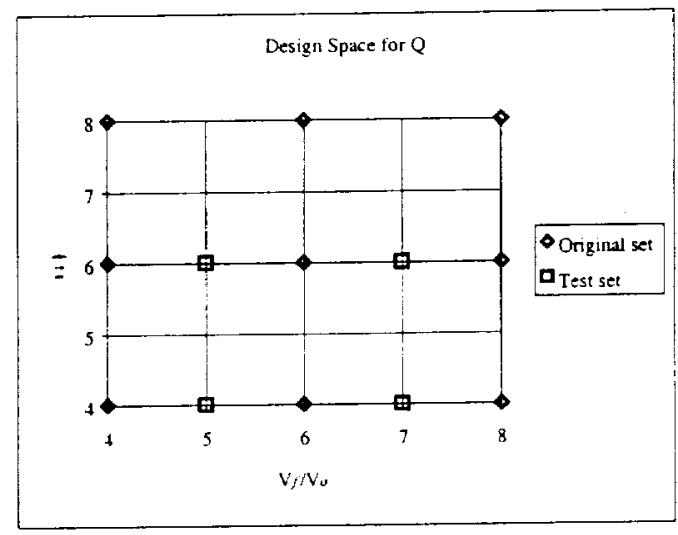

(b)

Figure 14. Design space for the shear co-axial injector. (a) $E R E$ ( 15 original points, 10 test points), (b) $Q(9$ original points, 4 test points).

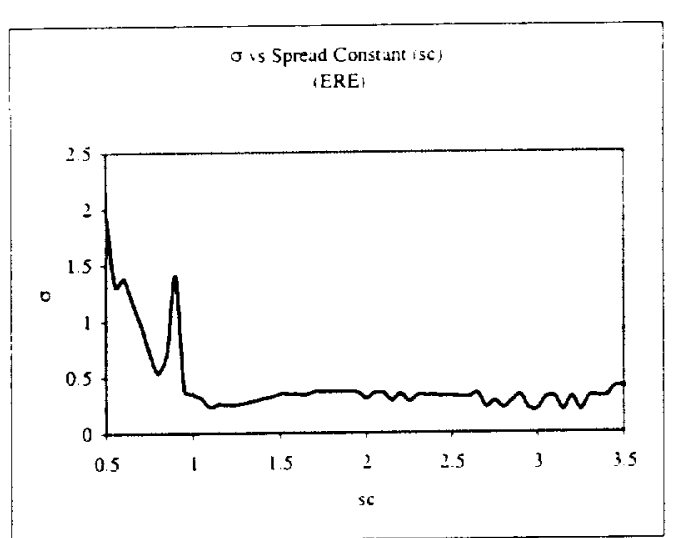

(a)

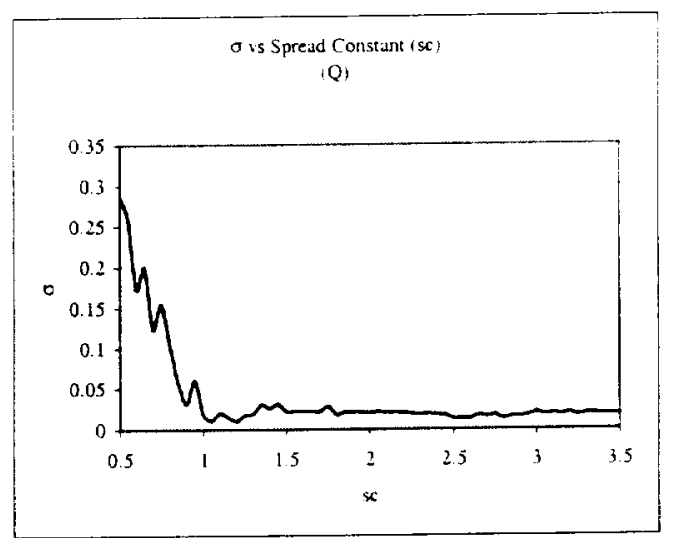

(b)

Figure 15. Comparison of $\sigma$ for different $N N$ designed with solverbe for shear co-axial gas-gas injector (a) $E R E(\%)$ and (b) $Q\left(\mathrm{Btu} / \mathrm{in}^{2}-\mathrm{sec}\right)$. 


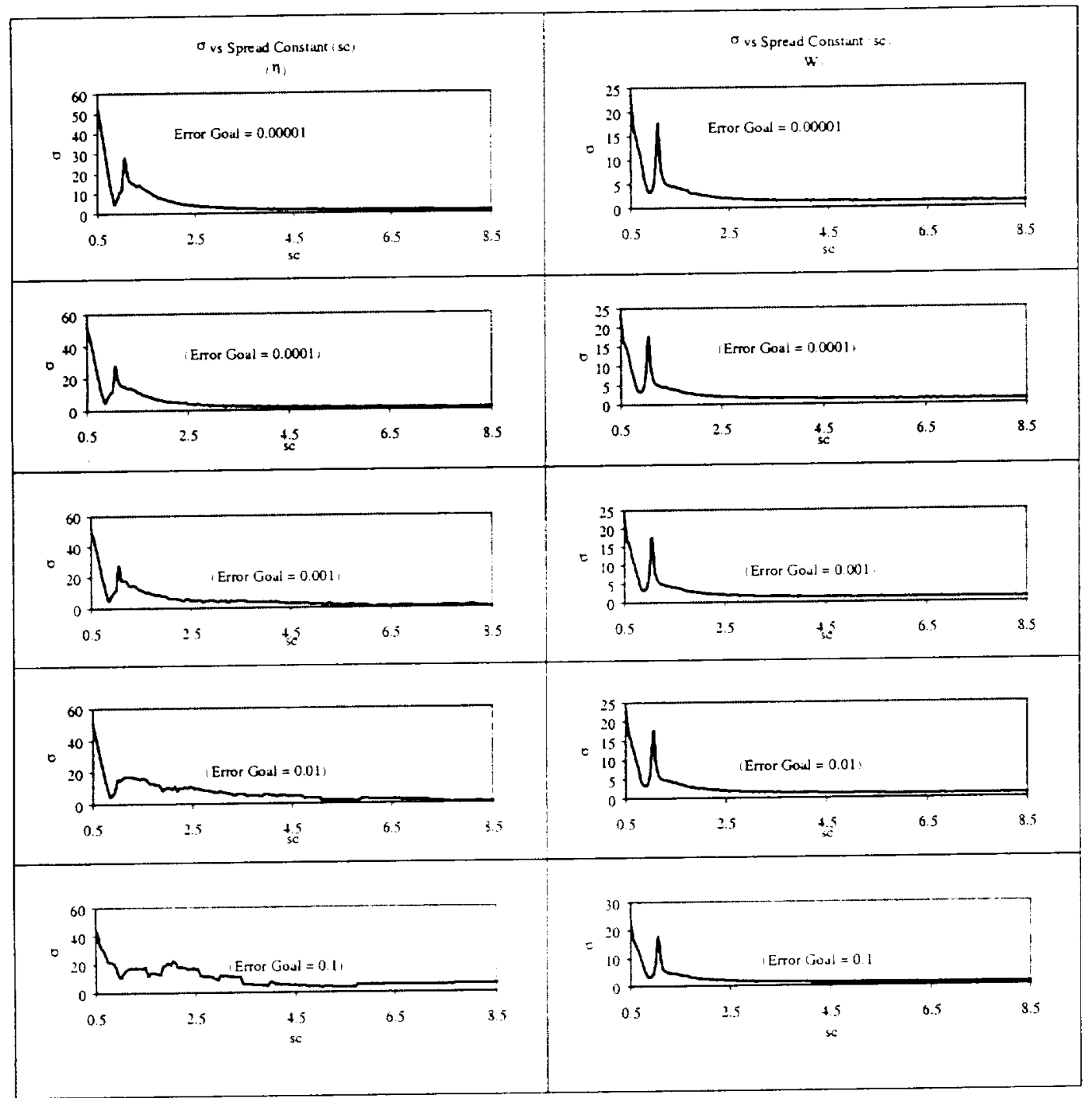

(a)

(b)

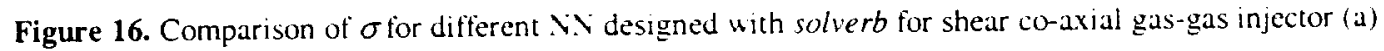
$E R E\left(C_{c}\right)$ and $(\mathrm{b}) Q\left(\mathrm{Bu} / \mathrm{in}^{2}-\mathrm{sec}\right)$. 

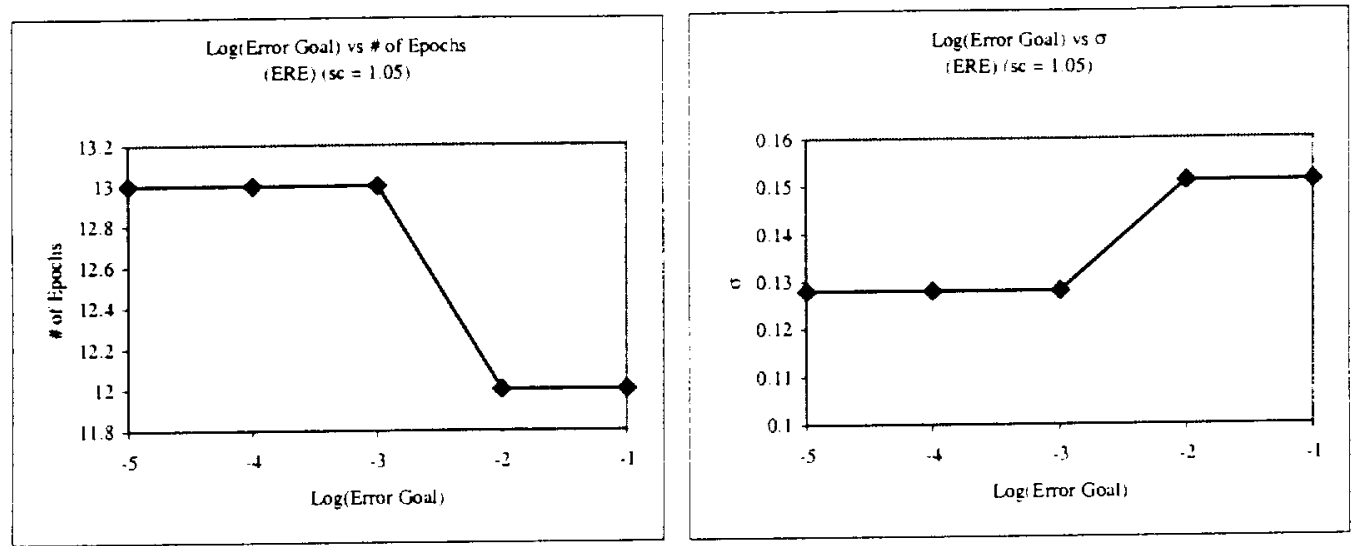

(a)
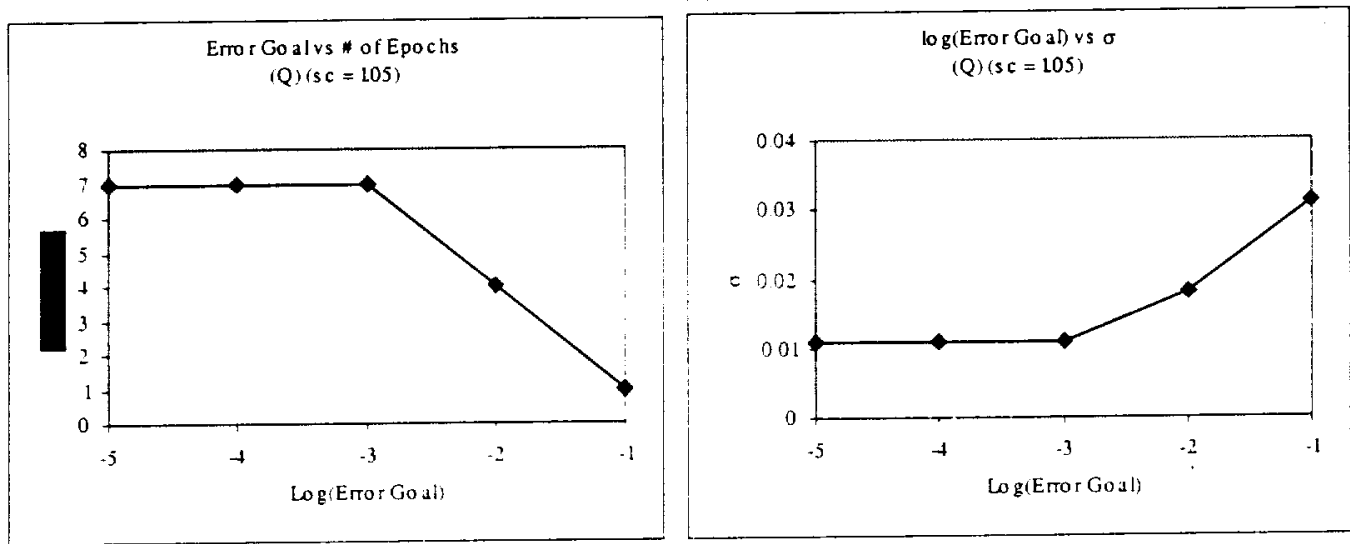

(b)

Figure 17. Comparison of Error goal vs. number of Epochs and $\sigma$ for networks trained with solverb for shear co-axial gas-gas injector (a) $E R E$ (\%), (b) $Q$ (Btu/in²-sec). 


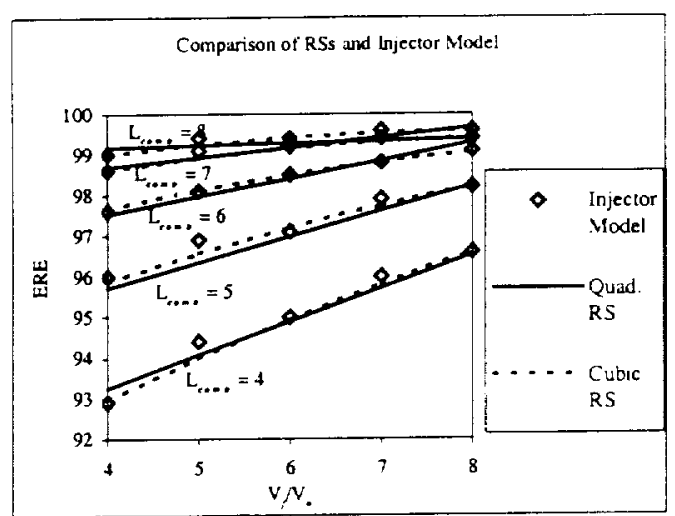

(a)

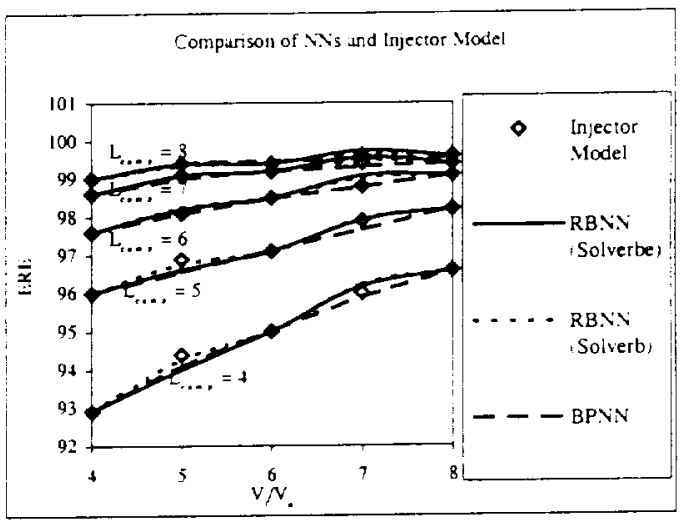

(b)

Figure 18. Predictive capabilities of various models for $E R E$ of the shear co-axial injector. (a) Polynomial (b) NN.

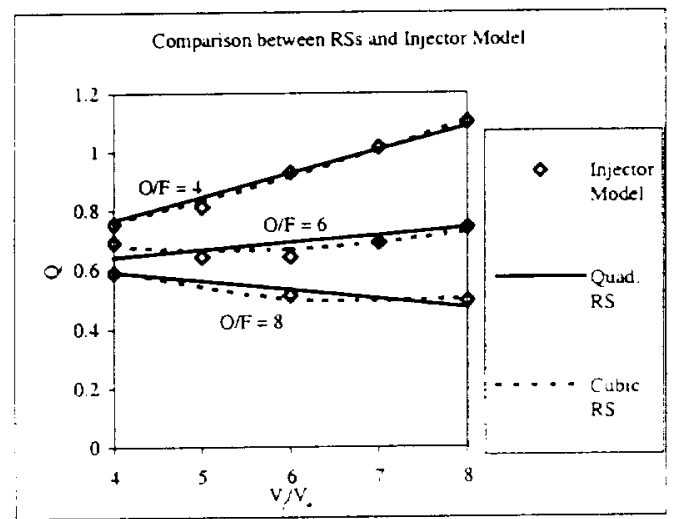

(a)

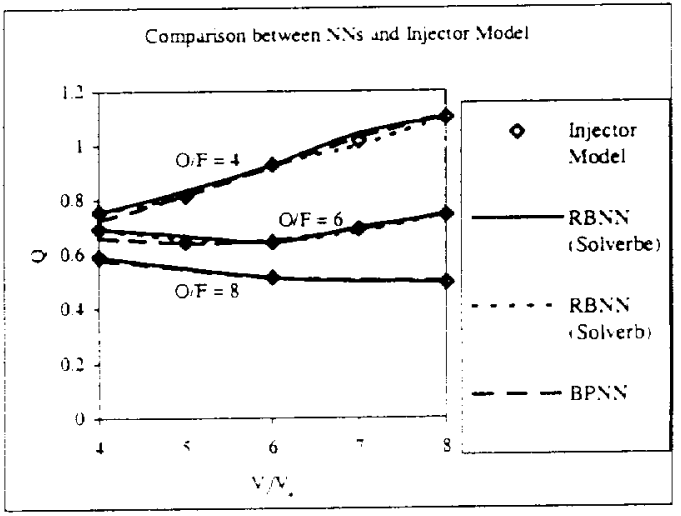

(b)

Figure 19. Predictive capabilities of various models for $Q$ of the shear co-axial injector. (a) Polymomial (b)

NN. 


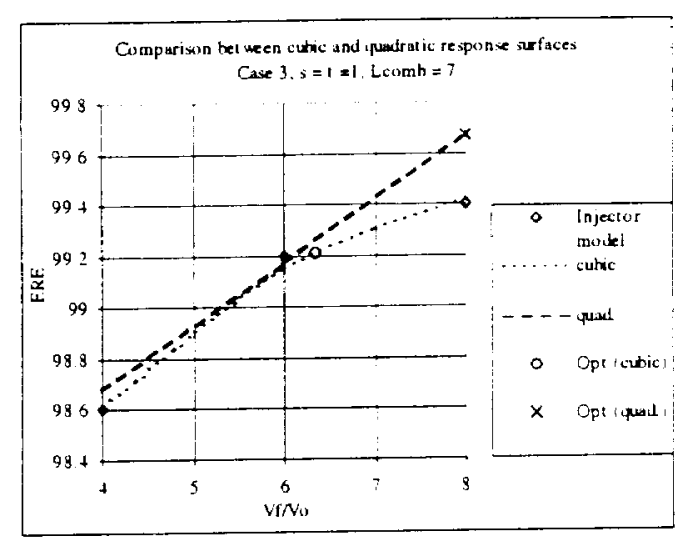

(a)

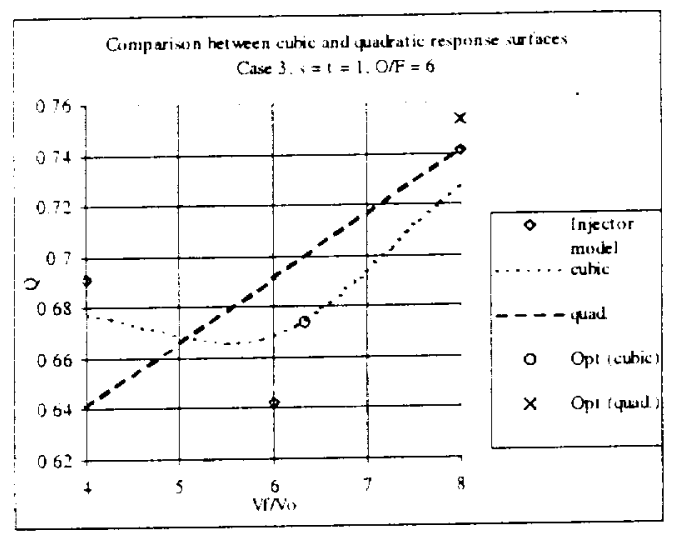

(b)

Figure 20. Comparison between cubic and quadratic response surface for case 3 of (a) $E R E$. (b) $Q$ for the shear co-axial injector. (NN and injector model data are the same points in the graph. Quadratic and cubic are predicted by polynomial-based RSM)

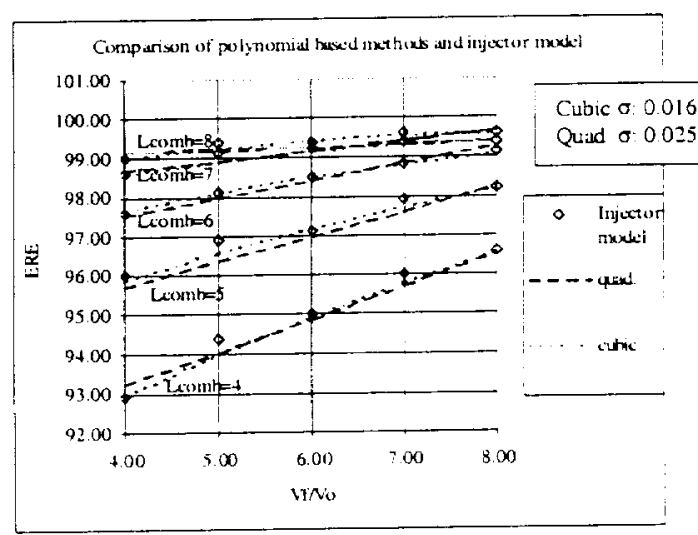

(a)

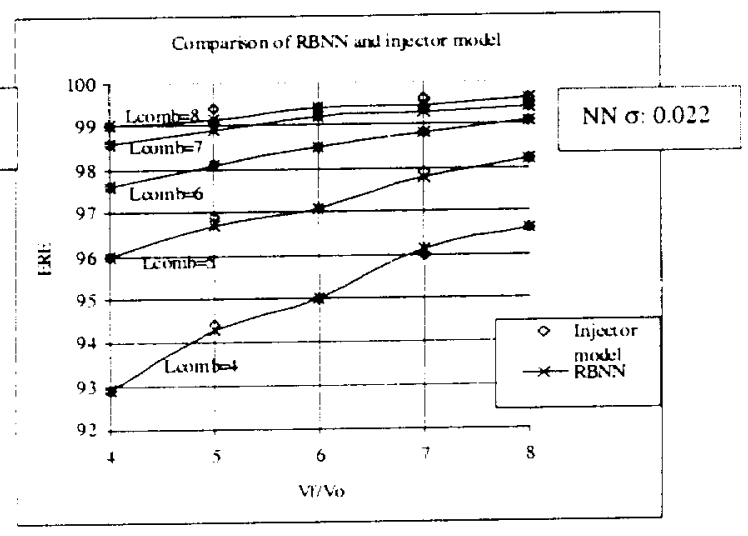

(b)

Figure 21. Assessment of predictive capabilities of (a) polynomial based method. (b) RBNN for ERE of the shear co-axial injector. ( 15 training/mapping points 10 test points) 


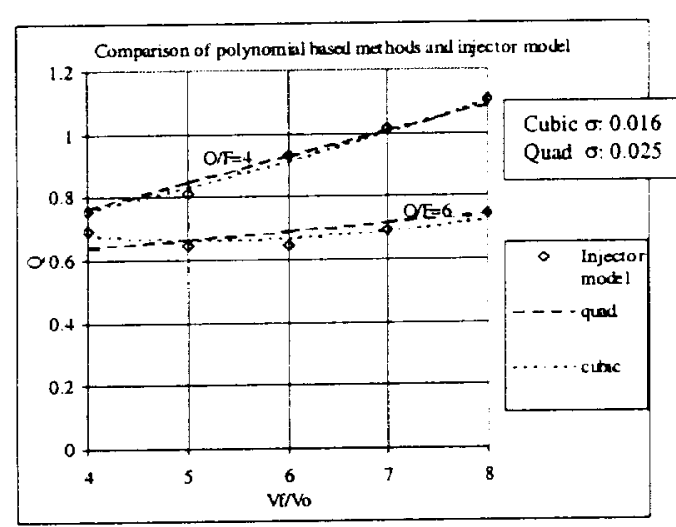

(a)

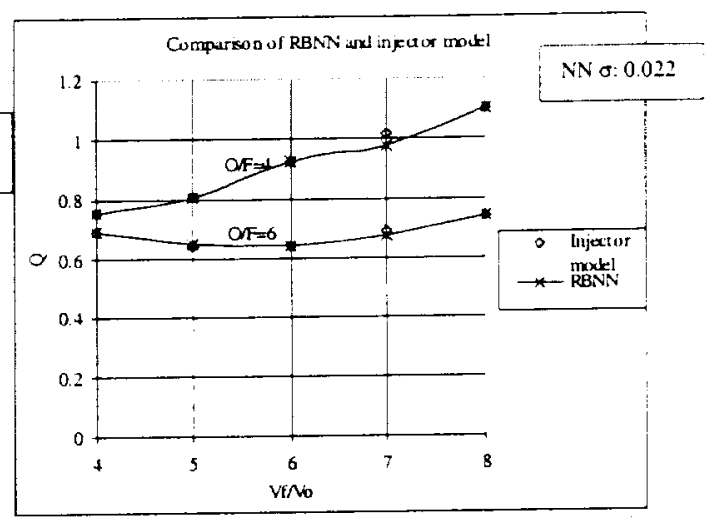

(b)

Figure 22. Assessment of performance of cubic and quadratic response surfaces for case 1 of (a) $E R E$ of the shear co-axial injector. (25 training/mapping points) (b) $Q$. (13 training/mapping points) \{Enhanced set includes Injector model data and RBNN predicted data. Quadratic and cubic are predicted by polynomialbased RSM

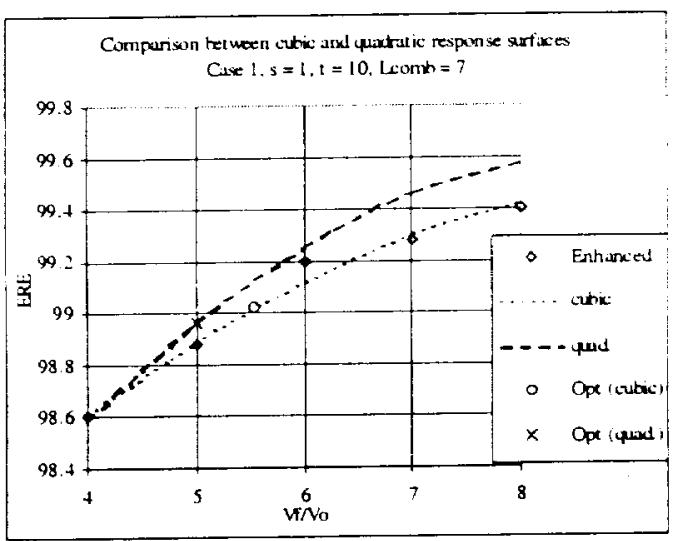

(a)

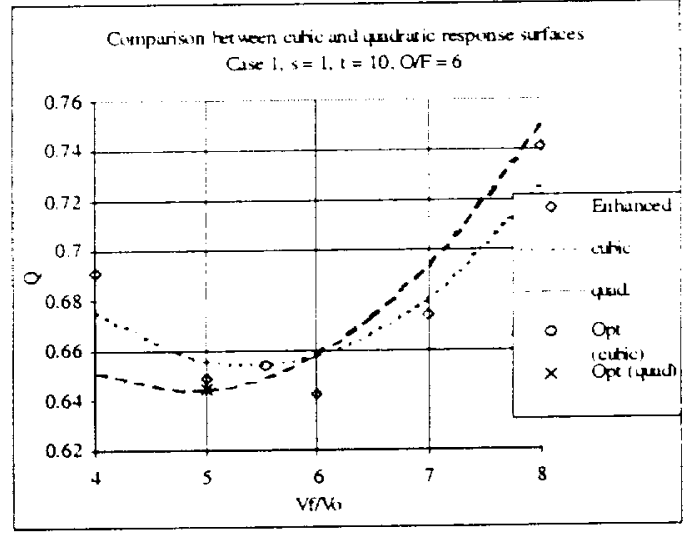

(b)

Figure 23. Assessment of performance of cubic and quadratic response surfaces for case $I$ of (a) $E R E$ for the shear co-axial injector. (25 training/mapping points) (b) $Q$. (13 training/mapping points) (Enhanced set includes Injector model data and RBNN predicted data. Quadratic and cubic are predicted by polynomialbased RSM 


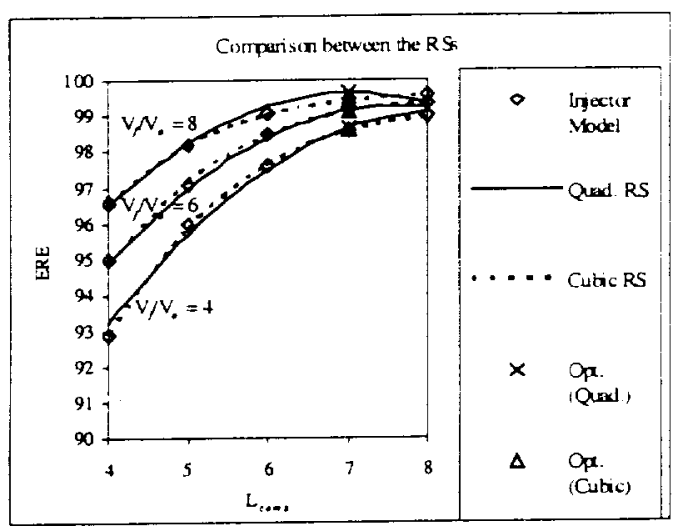

(a)

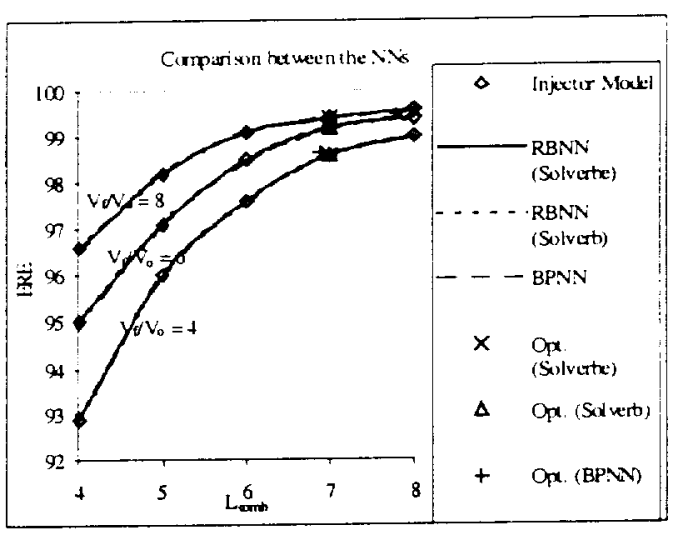

(b)

Figure 24. Performance of various models for $E R E$ of the shear co-axial injector. (a) RS's, (b) NN's.

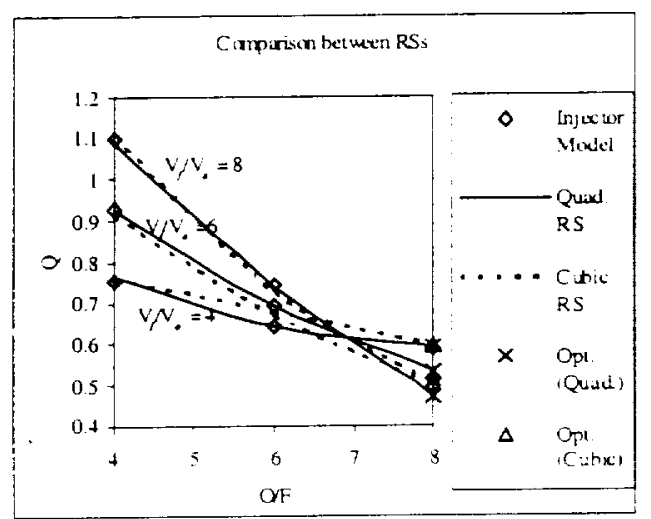

(a)

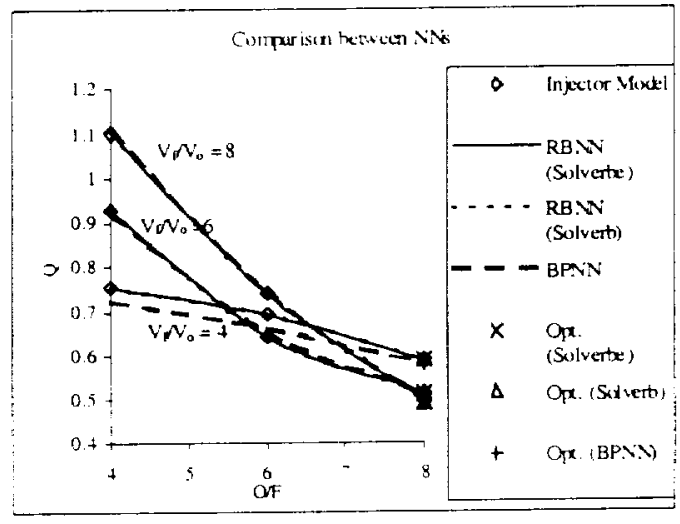

(b)

Figure 25. Performance of various models for $Q$ of the shear co-axial injector. (a) RS's, (b) NN's. 


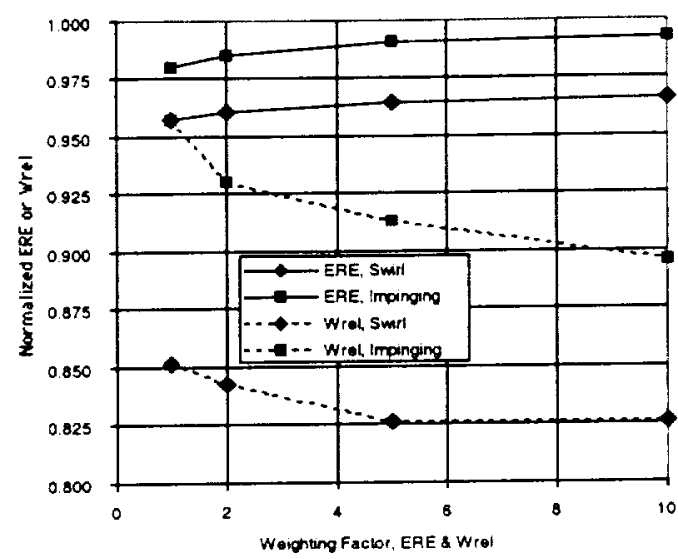

Figure 26. Performance and weight trends for swirl and impinging elements.

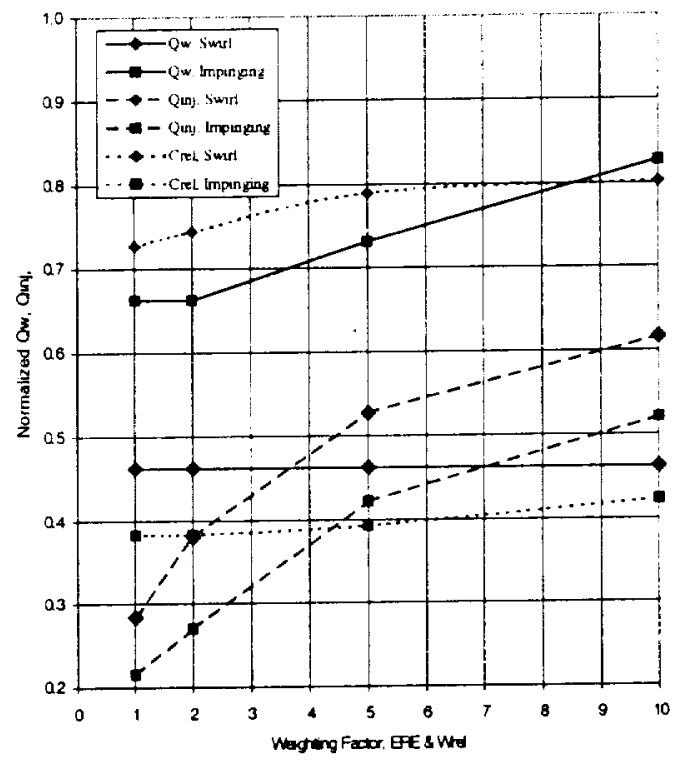

Figure 27. Heat flux and cost trends for swirl and impinging elements. 

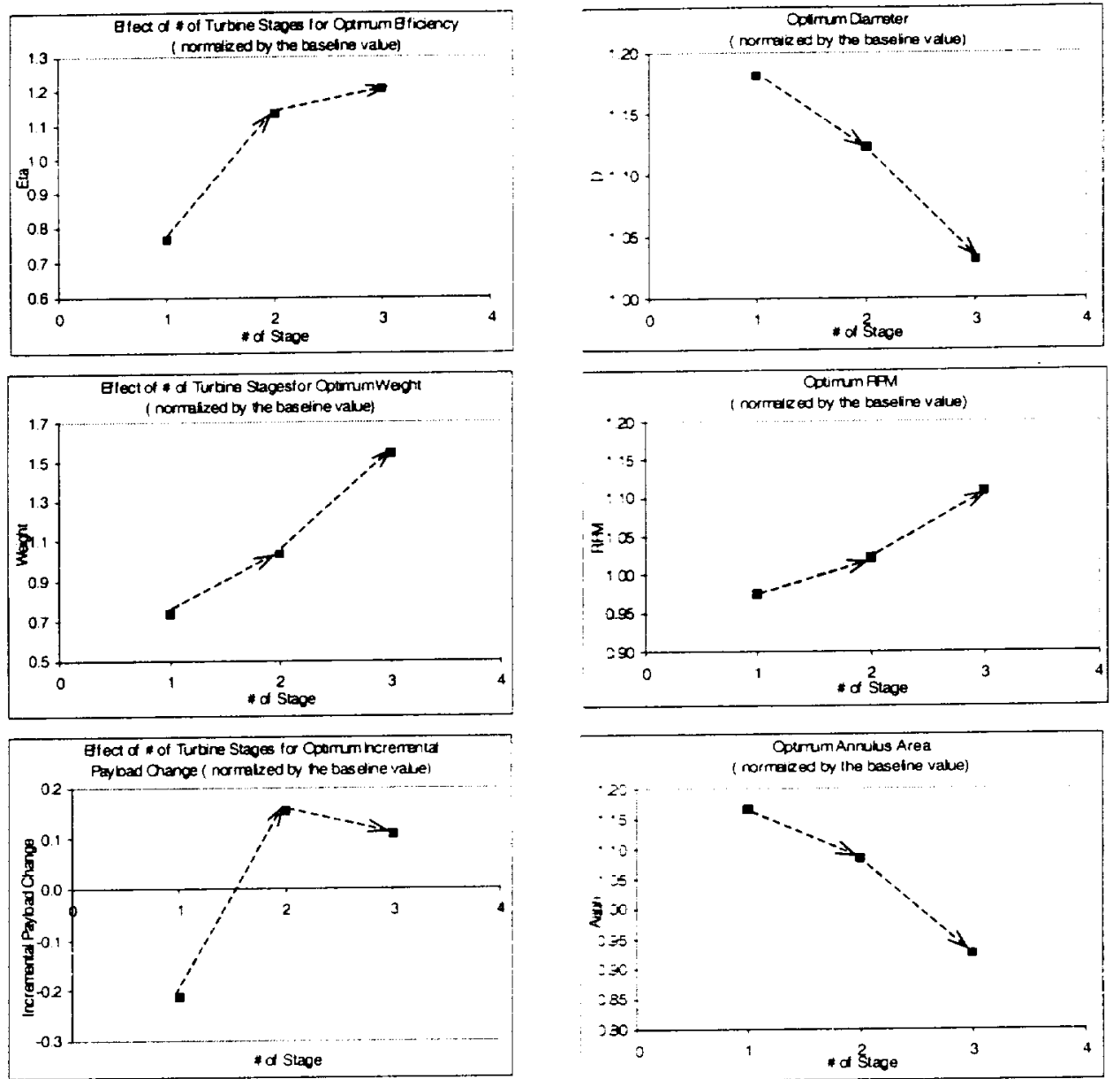

Figure 28. Effect of the number of turbine stage on optimum design parameters; $D, R P M$. and $A_{u n n}$ and optimum output parameters: $\eta, W$, and spay calculated for spay-based optimization (All geometric design variables and output parameters are normalized by the baseline values)

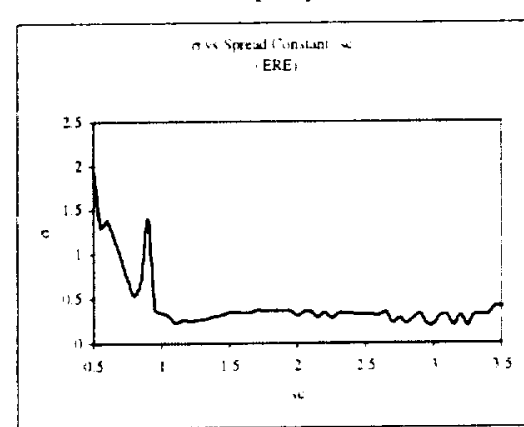

(a)

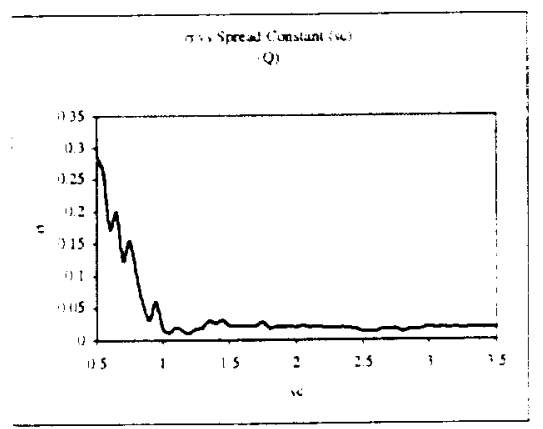

(b)

Figure 29. Comparison of $\sigma$ for different $N V$ designed with solverbe for single-stage supersonic turbine (a)

$$
\eta(t) \text { and (b) Wilbs). }
$$




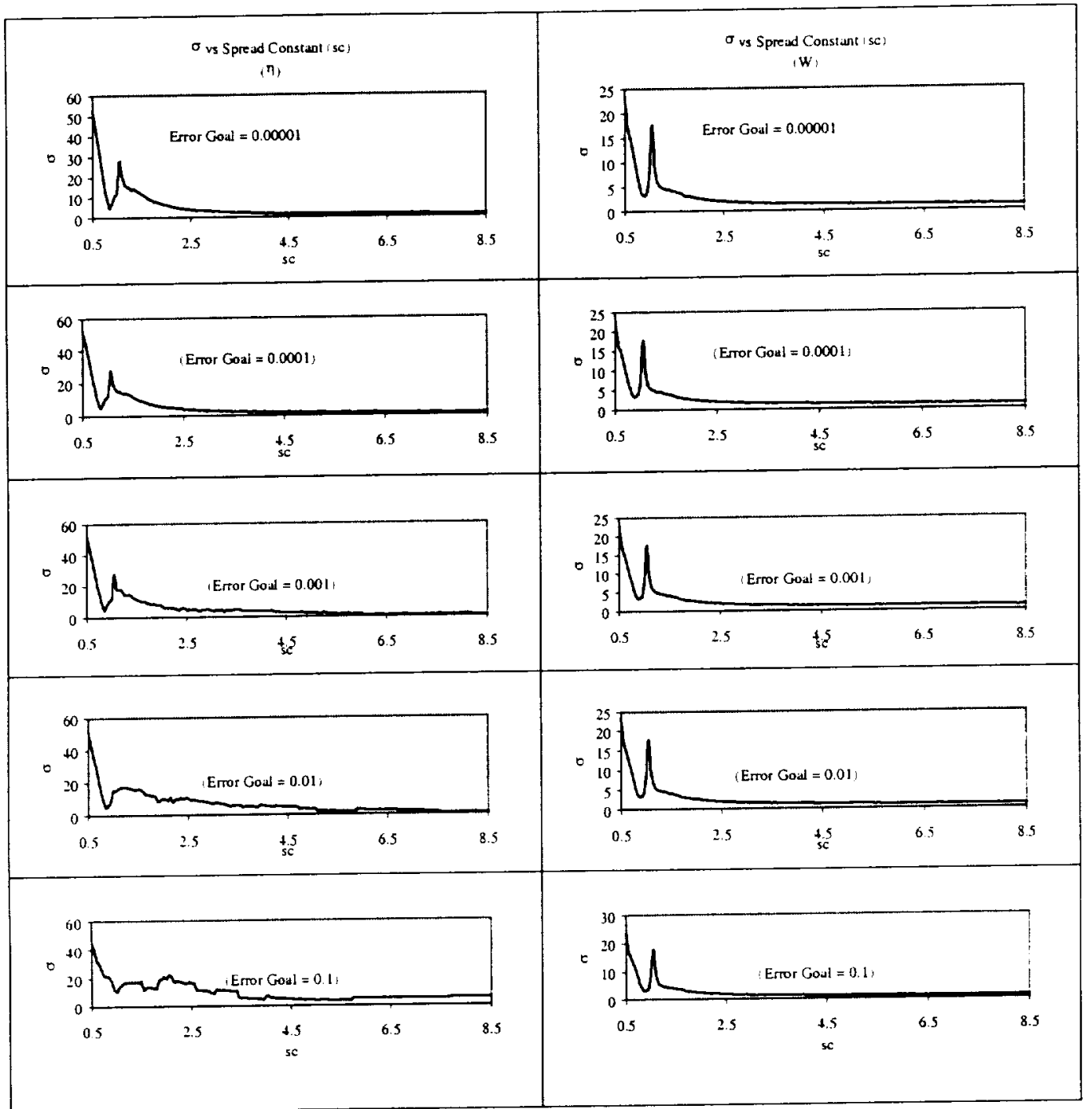

(a)

(b)

Figure 30. Comparison of $\sigma$ for different $\mathrm{NN}$ designed with solverb for single-stage supersonic turbine (a) $\eta(\%)$ and (b) $W(\mathrm{lbs})$ 

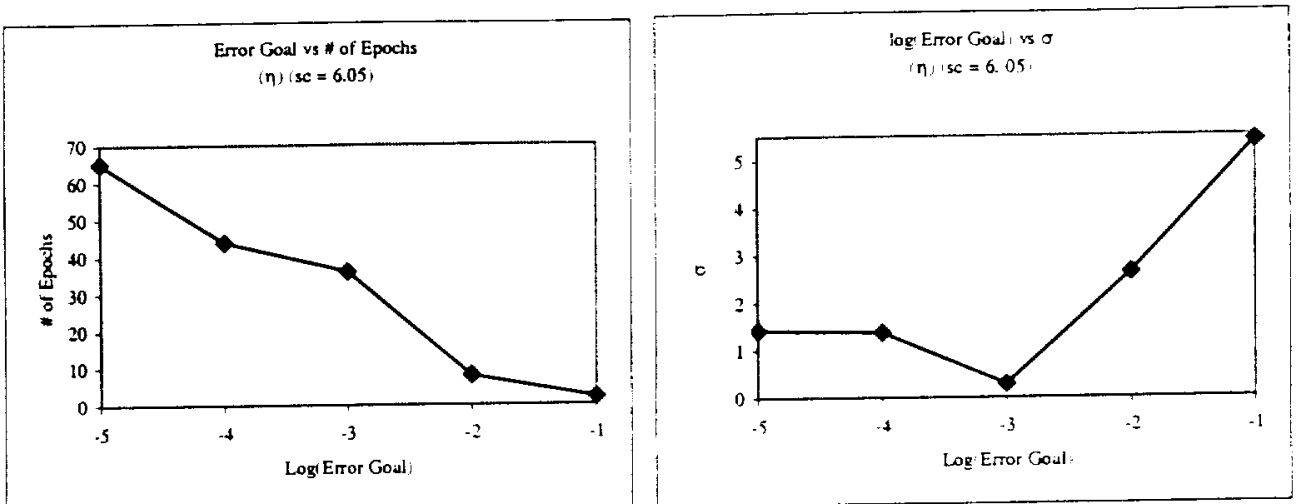

(a)
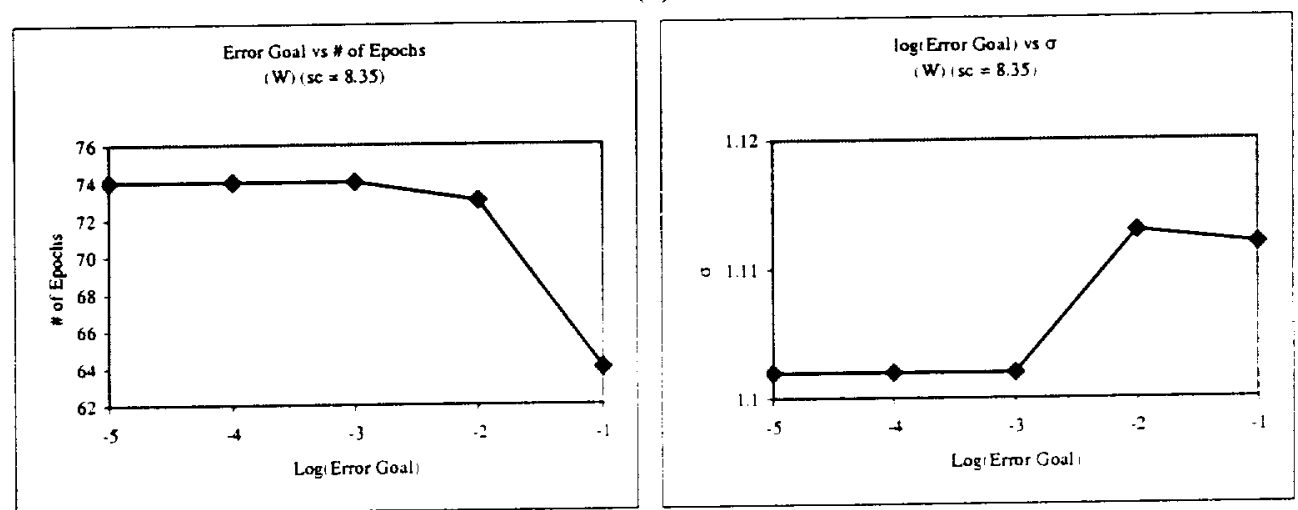

(b)

Figure 31. Comparison of error goal vs. number of epochs and $\sigma$ for networks trained with solverb for single-stage supersonic turbine (a) $\eta(\%)$ and (b) $W$ (lbs) 


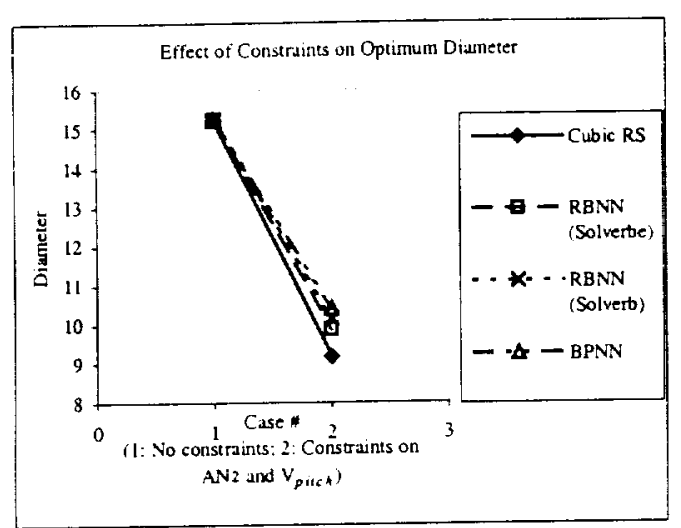

(a)

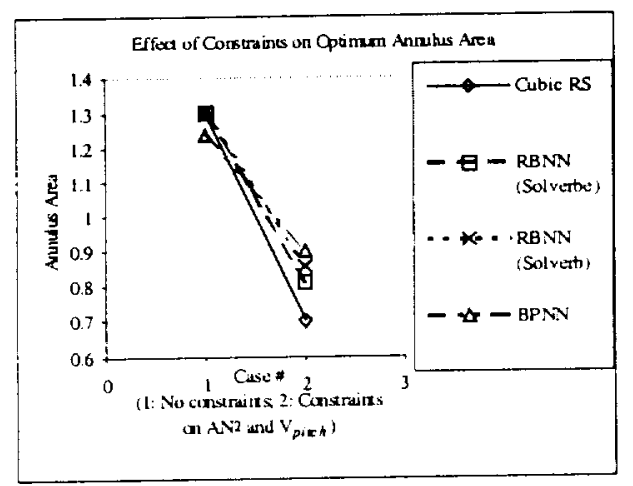

(c)

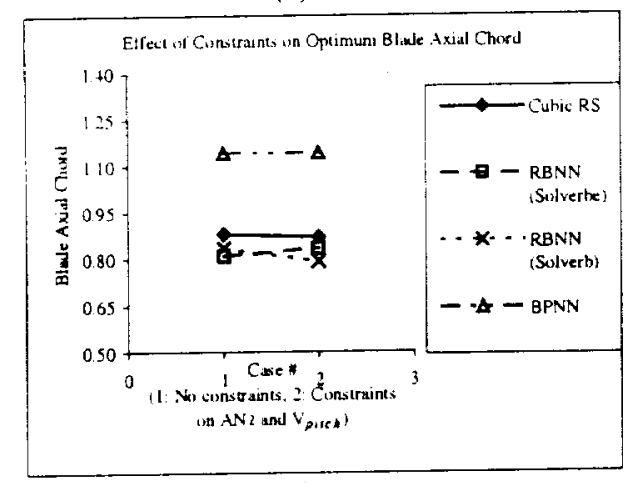

(e)

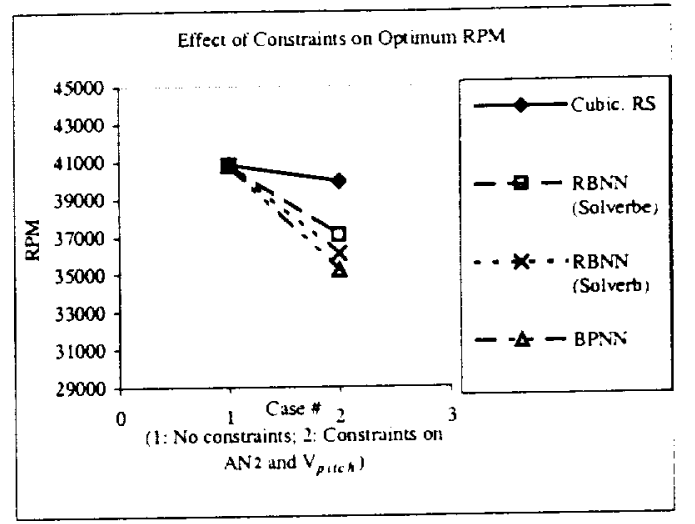

(b)

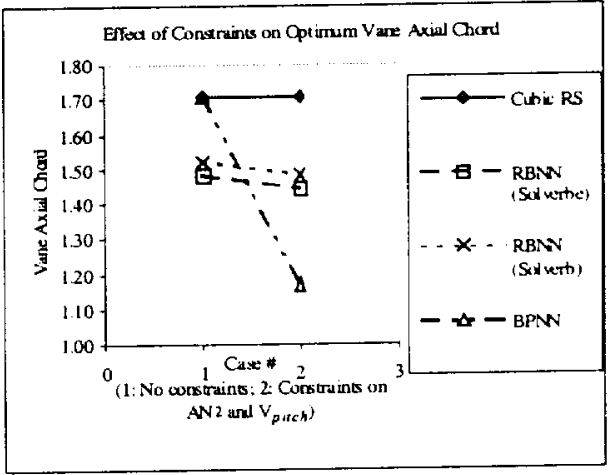

(d)

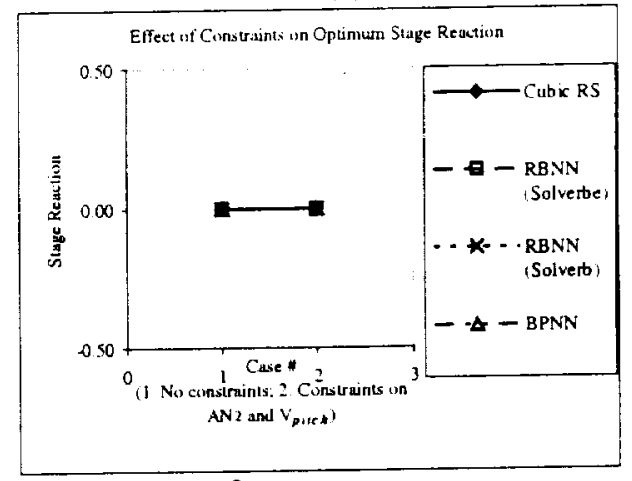

(f)

Figure 32. Effect due to presence (case 1) or lack of constraints (case 2) on design variables. (a) optimum diameter, $D$ (in.), (b) optimum $R P M$, (c) optimum annulus Area, $A_{a n n}\left(\right.$ in. $^{2}$ ), (d) optimum vane axial chord, $C_{V}$ (in.), (e) optimum blade axial chord, $C_{b}\left(\right.$ in.), (f) optimum stage reaction, $K_{\mathrm{r}}(\%)$. 


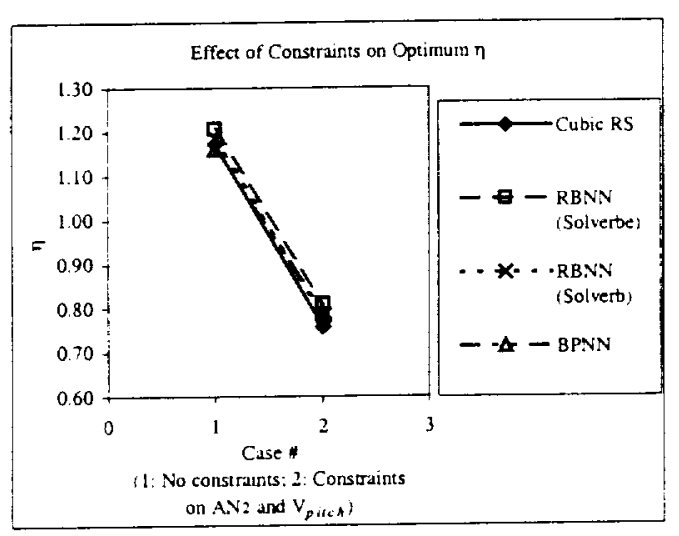

(a)

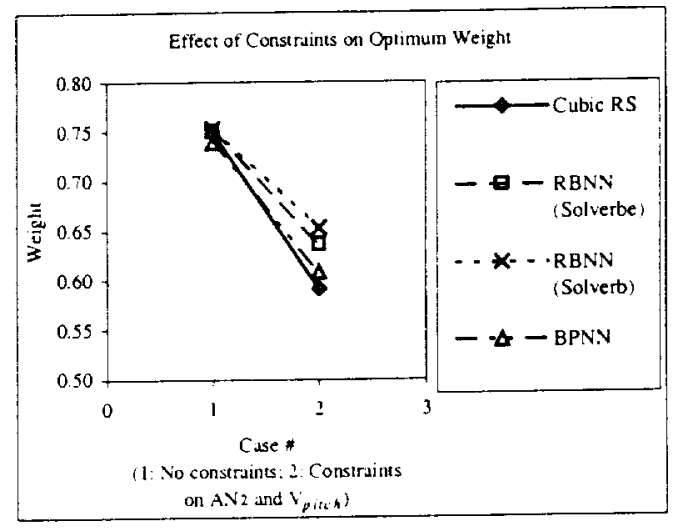

(b)

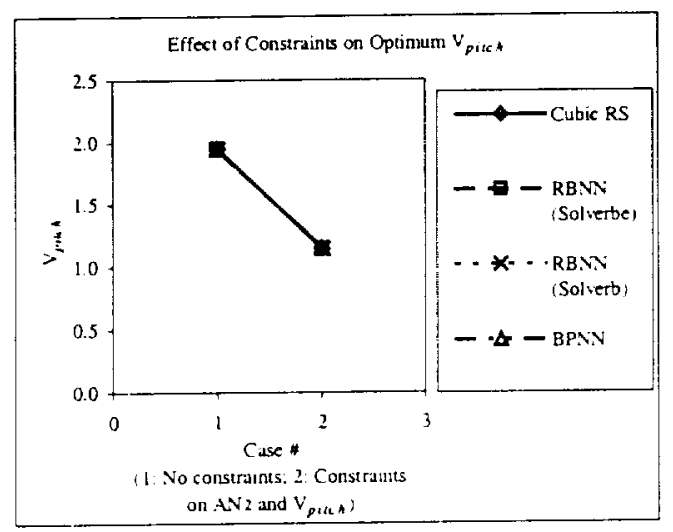

(c)

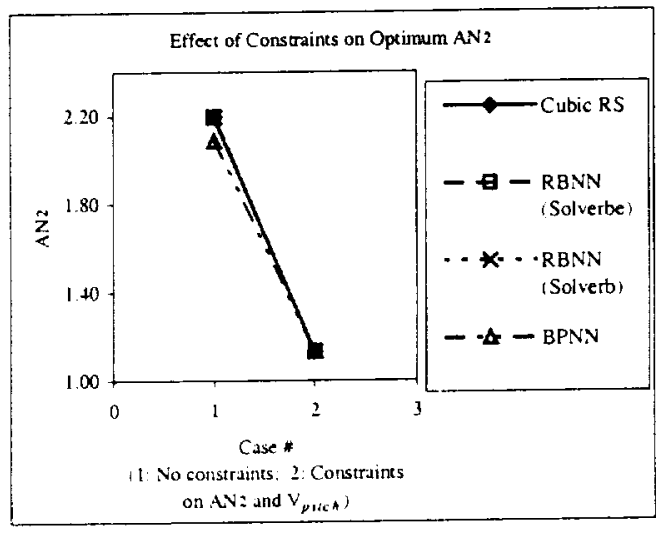

(d)

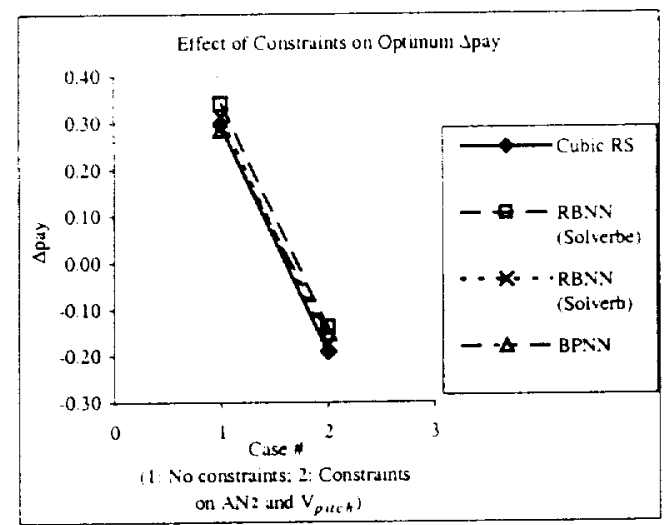

(e)

Figure 33. Effect due to presence (case 1) or lack of constraints (case 2) on objective functions. (a)

Optimum Efficiency, $\eta$, (b) Optimum Weight, $W$ (lbs), (c) Optimum pitch speed, $V_{\text {puch }}$ (in. /sec), (d)

Optimum $\left(A_{t n n} \times R P M\right), A N^{2}\left(\right.$ in $\left.^{2} \mathrm{rpm}^{2}\right)$. (e) Optimum Incremental Payload. Apay (bs). 


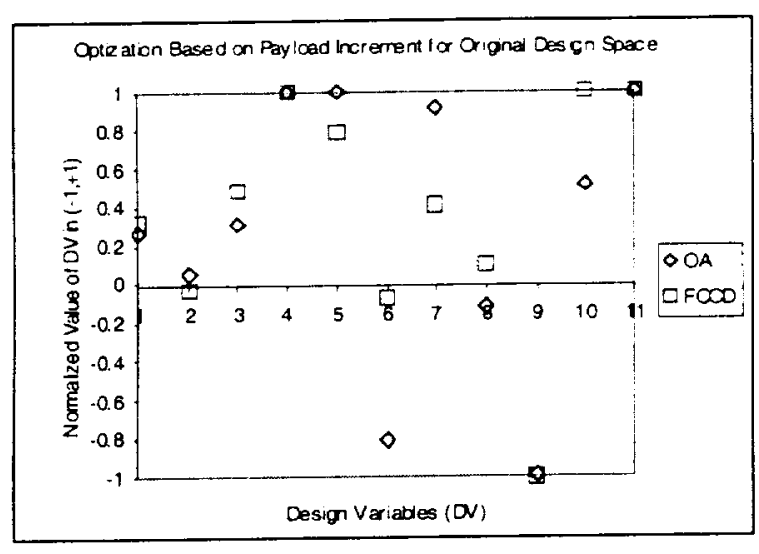

(a) Original Design Space

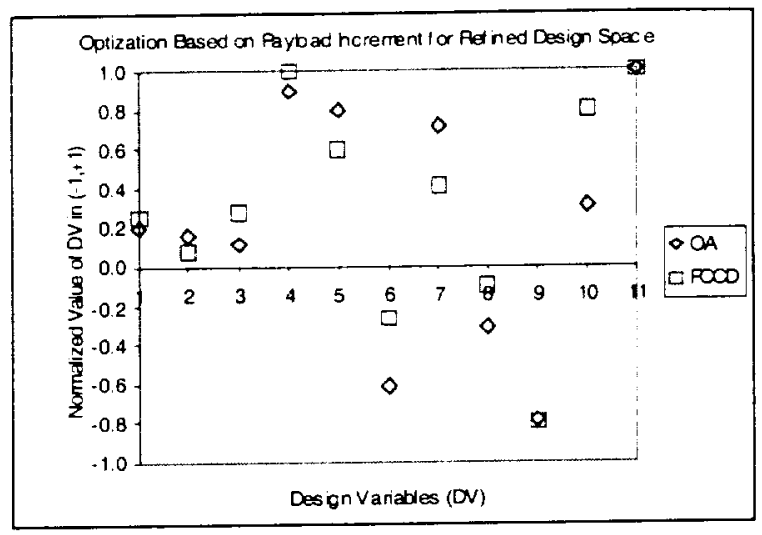

(b) Refined Design Space

Figure 34. Comparison of the design variables for optimization based on payload increment ( $\Delta$ pay) using 1990-data (FCCD) and 249-data (OA) for both original design space and refined design Space (DV\#l:D,

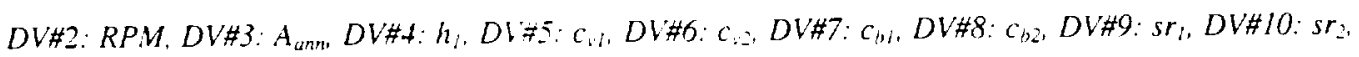
and DV\#11: $w_{1 /}$ ). Both designs are satisfactory, demonstrating that there exist multiple optimum designs.

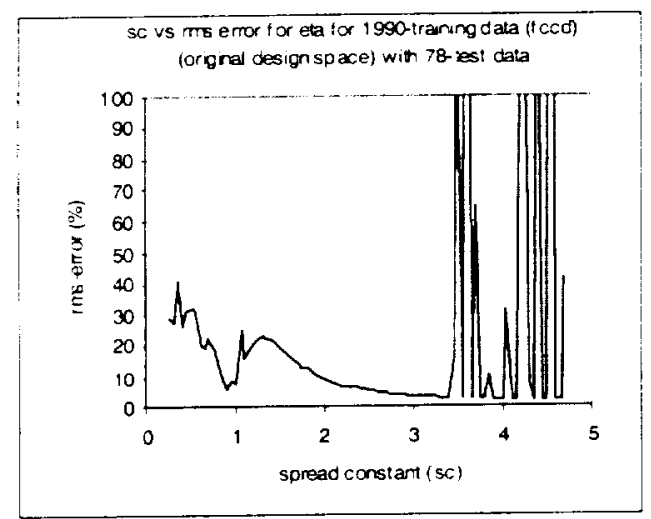

(a)

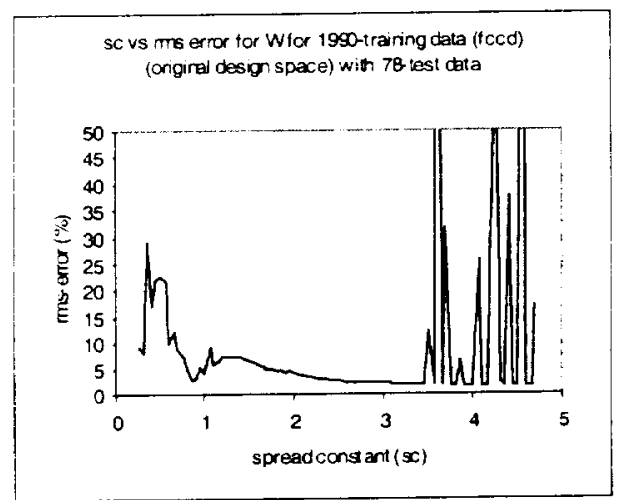

(b)

Figure 35. Effect of spread constant isc i on training rms-error $\left(\sigma_{u}\right)$ of (a) $\eta$ and (b) $W$ for preliminary design of 2-stage turbine for original design space using 1990-training data for solverbe RBNN 


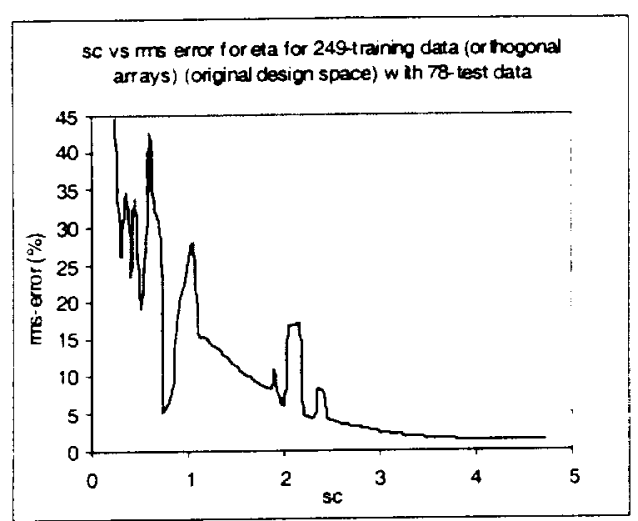

(a)

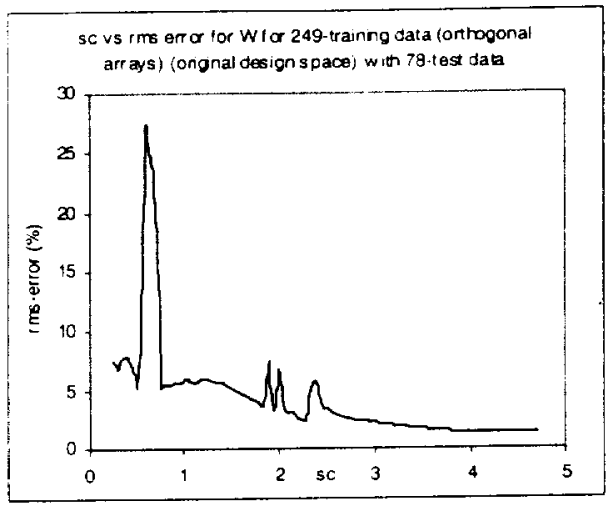

(b)

Figure 36. Effect of spread constant (sc) on training rms-error $\left(\sigma_{a}\right)$ of (a) $\eta$ and (b) $W$ for preliminary design of 2-stage turbine for original design space using 249-training data for solverbe RBNN

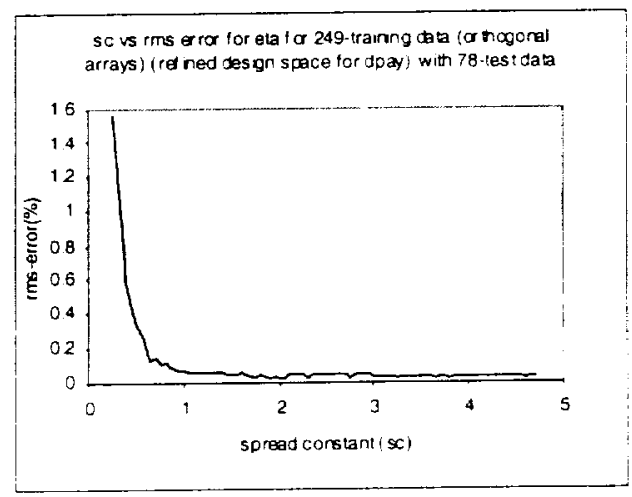

(a) for $\eta$ (spay optimization)

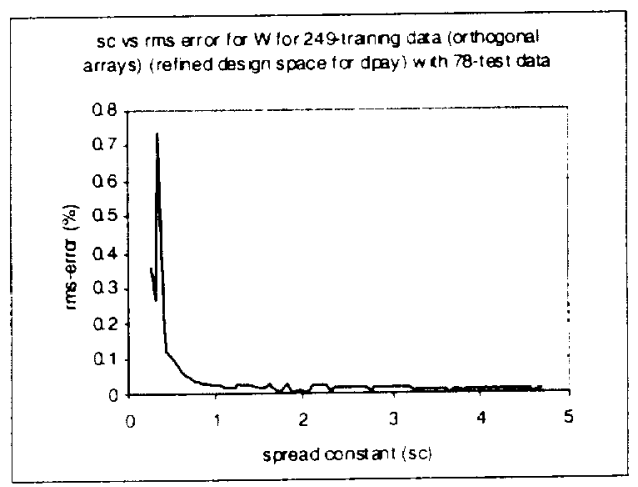

(b) for W ( $\Delta$ pay optimization)

Figure 37. Effect of spread constant (sc) on training rms-error $\left(\sigma_{2}\right)$ of for preliminary design of 2-stage turbine for refined design space using 249-training data (OA) for solverbe, RBNN. 

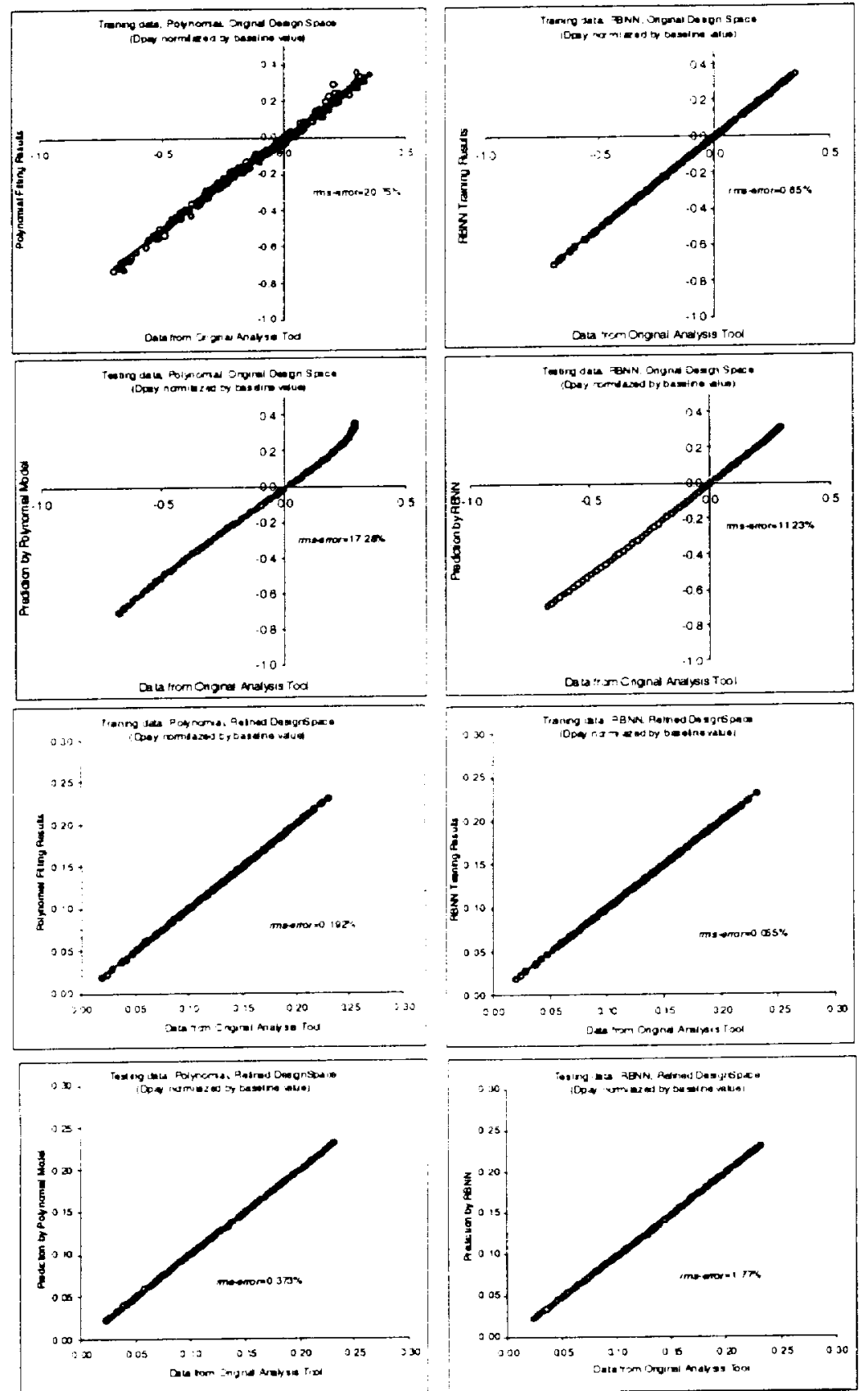

Figure 38. Comparison of $\mathrm{VN}$ and polynomial-based representations for 2-stage supersonic turbine. Plotted are the original and predicted values of 4 pay. A perfect fit will result in a $45^{\circ}$ line. The training and testing data are selected based on the orthogonal arrays with the D-optimal criterion. There are 11 design variables, 249 training data (OA), and 78 testing data in both original and refined design spaces. (The values for $4 p a y$ are normalized by the baseline value) 


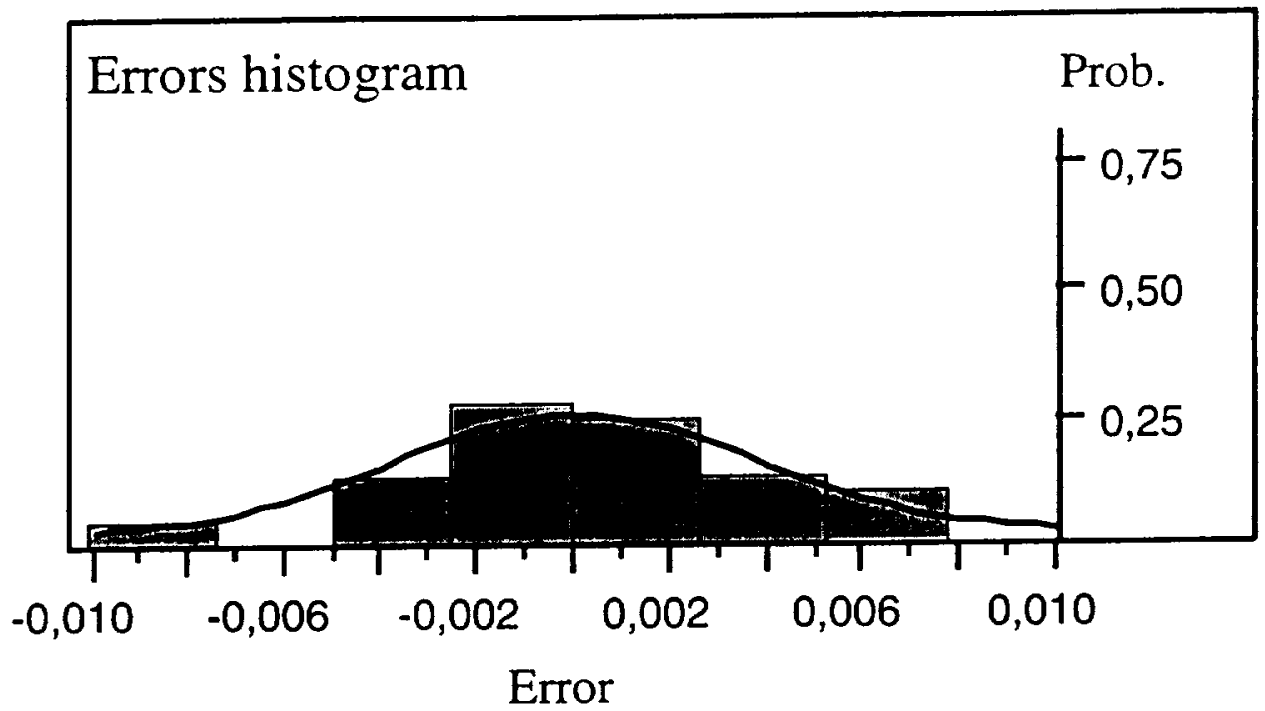

Figure 39. Distribution of response surface errors at sampling points and the corresponding normal distribution curve (same mean and variance).

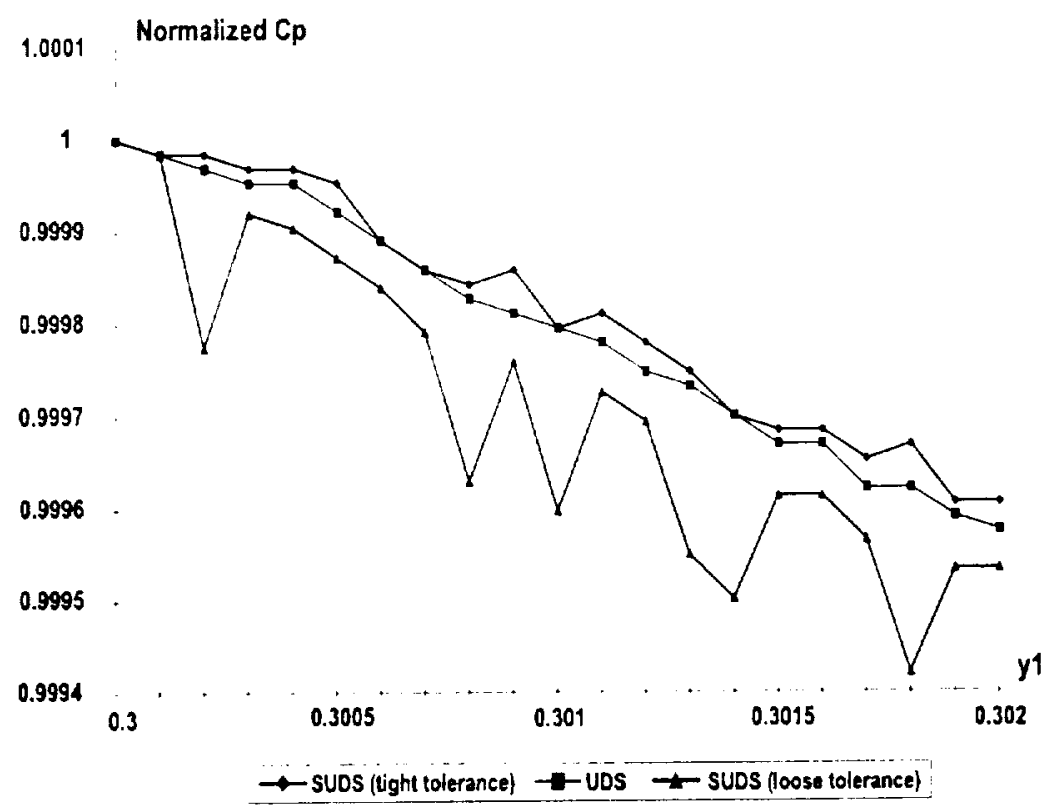

Figure 40. Normalized $C_{p}$-values along a straight line in the design space. The results are for two different differencing schemes and two different residual levels used as convergence criterion. (UDS: upwind differencing scheme and SUDS: second-order upwind differencing scheme) 


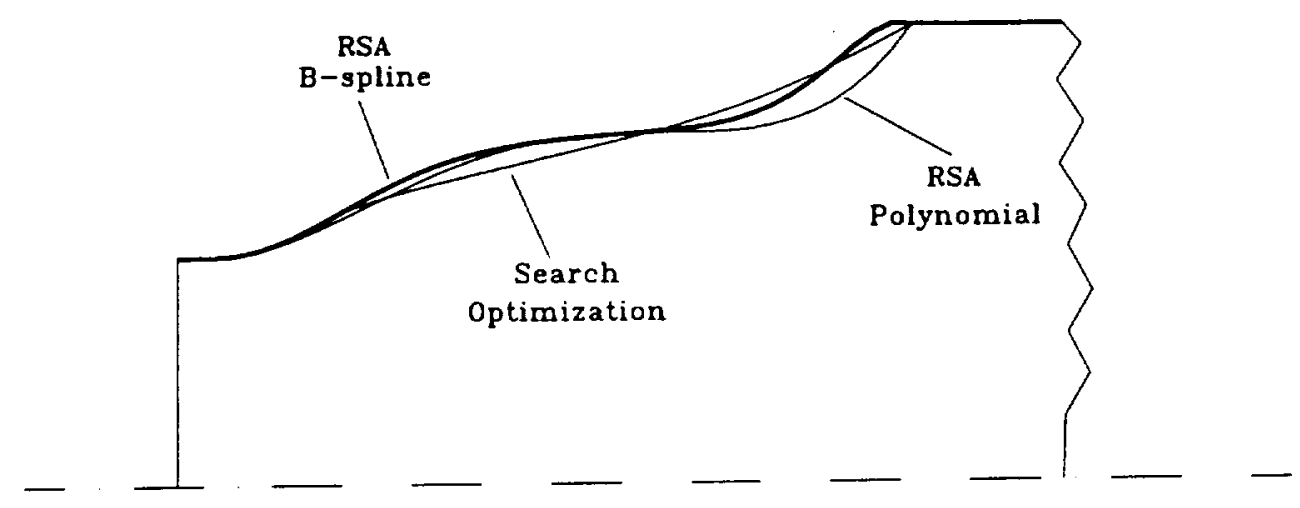

Figure 41. Comparison of optimum wall shapes using polynomial and B-spline representations, respectively.

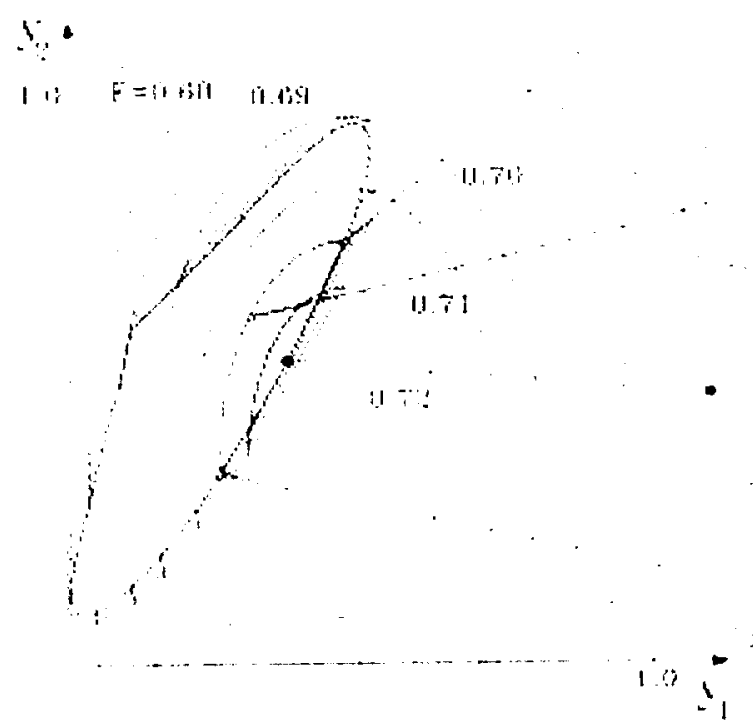

Figure 42. Contour plot of response surface for diffuser design. Solid circle indicates the optimal region.

The hatched part of the feasible space comprises designs with pertormance within $1 \%$ of the optimal.

Corresponding shapes are indicated to the right. The results indicate that multiple design points meet the goal. 


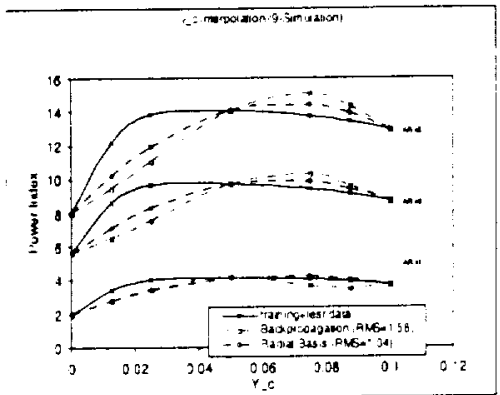

(a) 9-Simulation Training

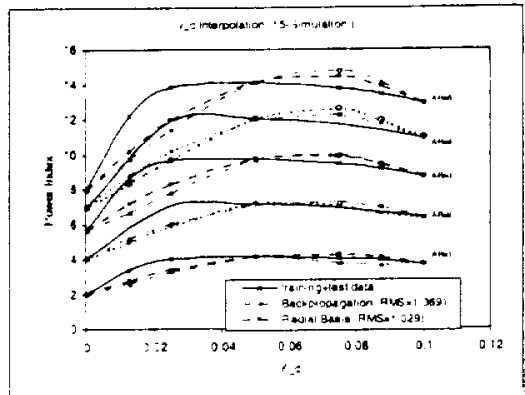

(b) 15-Simulation Training

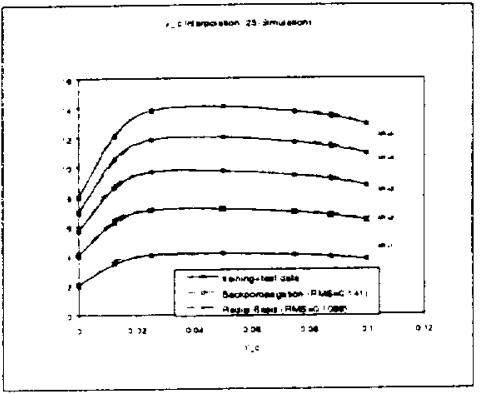

(c) 25-Simulation Training

Figure 43. Comparison of radial basis network with back-propagation network results for 3-D wing model

(for $y_{c}$ interpolation) (Design parameters: $s c=1.175$, error goal=0.1 for radial-basis, and \#of neurons=4.

error goal=0.01 for back-propagation)

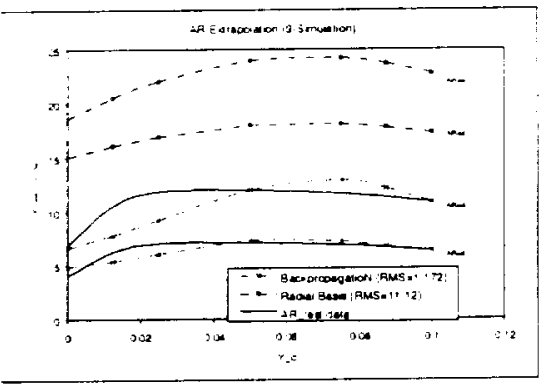

(a) 9-Simulation Training

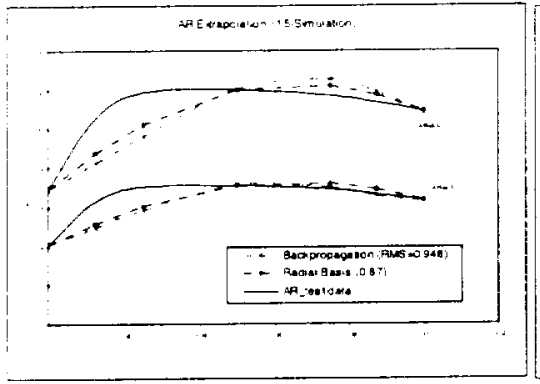

(b) 15-Simulation Training

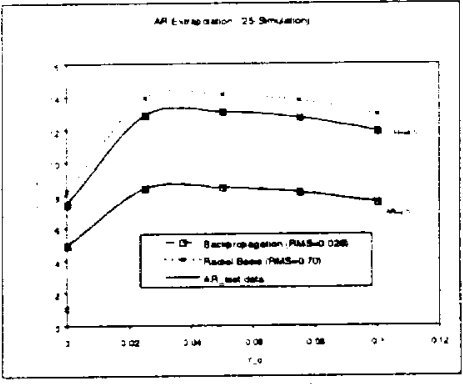

(c) 25 -Simulation Training

Figure 4. Comparison of radial basis network with back-propagation network results for 3-D wing model ( for $A R$ interpolation) (Design parameters: $s c=1.175$, crror goal=0.1 for radial-basis, and $\#$ of neurons $=4$,

error goal=0.01 for back-propagation )

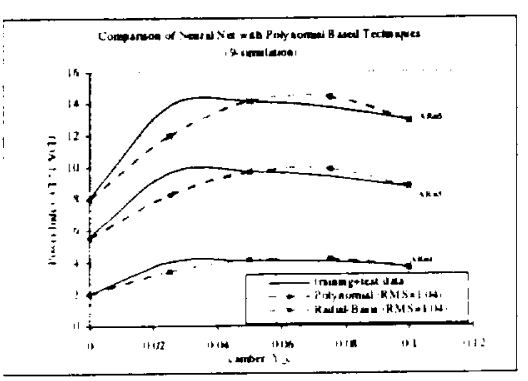

(a) 9-Simulation Training

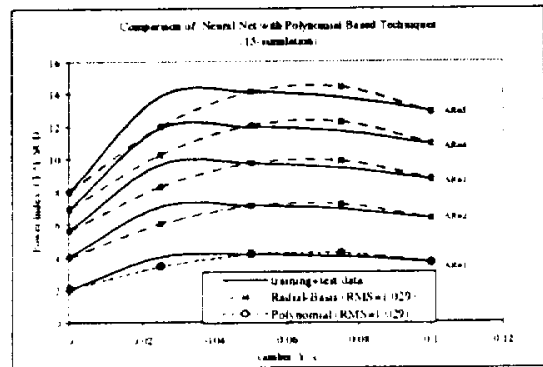

(b) 15-Simulation Training

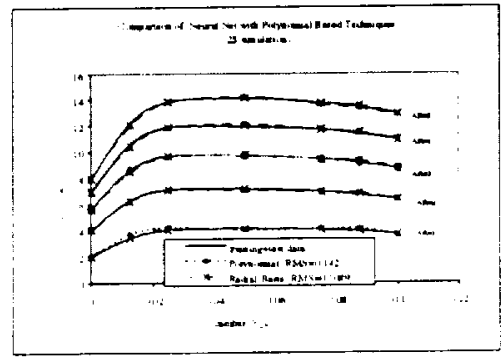

(c) 25-Simulation Training

Figure 45. Comparison of radial basis NN results with polynomials for 3-D wing model (Design

parameters: $s c=1.175$. error goal $=0.1$ for radial-basis networks) 


\section{TABLES}

Table 1. Comparison of NN and polynomial-based response surface (RS) techniques

\begin{tabular}{|c|c|c|c|}
\hline & $\begin{array}{l}\text { NN-based } \\
\text { RSM }\end{array}$ & $\begin{array}{l}\text { Polynomial- } \\
\text { based RSM }\end{array}$ & Comments \\
\hline $\begin{array}{l}\text { Computational Effort } \\
\text { and Cost }\end{array}$ & Disadvantage & Advantage & $\begin{array}{l}\text { Finding the weights associated with the neurons is a } \\
\text { non-linear regression process for all of the } \\
\text { types other than RBNN. Whereas, finding the } \\
\text { polynomial coetficients requires solution of a linear } \\
\text { set of equations. } \\
\text { The cost increases if the regression process is non- } \\
\text { linear which makes } \mathrm{NN} \text { 's other than RBNN more } \\
\text { expensive than polynomials. }\end{array}$ \\
\hline Noise & Disadvantage & Advantage & $\begin{array}{l}\text { Ability of filtering noise from experimental data is } \\
\text { possible with polynomial-based RSM. However, if } \\
\text { the number of neurons used to design the } \mathrm{NN} \text { is not } \\
\text { the same as the data, then, by detinition, filtering is } \\
\text { also possible for NN-based RS.M. }\end{array}$ \\
\hline $\begin{array}{l}\text { Handling complex } \\
\text { functions }\end{array}$ & Advantage & Disadiantage & $\begin{array}{l}\text { NN's are more suitable for multi-dimensional } \\
\text { interpolation of data that lack structure since they } \\
\text { are much more tlexible in functional form } \\
\text { especially when dealing with design in the context } \\
\text { of unsteady tlows, partial and/or complete data sets. }\end{array}$ \\
\hline
\end{tabular}

Table 2. Literature review on NN and polynomial-based RS techniques comparison

\begin{tabular}{|c|c|c|c|c|c|c|c|c|}
\hline Authors & $\begin{array}{l}\text { No of } \\
\text { Data }\end{array}$ & $\begin{array}{l}\text { No of } \\
\text { Input }\end{array}$ & $\begin{array}{l}\text { No of } \\
\text { Output }\end{array}$ & $\begin{array}{l}\text { NN Type } \\
\text { (2-laver) }\end{array}$ & $\begin{array}{l}\text { Activation } \\
\text { Function }\end{array}$ & \multicolumn{2}{|c|}{$\begin{array}{l}\text { No of } \\
\text { Neurons }\end{array}$} & $\begin{array}{c}\text { Polynomial } \\
\text { Degree }\end{array}$ \\
\hline \multirow{4}{*}{$\begin{array}{l}\text { Carpenter \& } \\
\text { Barthelemy } \\
{[4]}\end{array}$} & 36 & 2 & 1 & \multirow[t]{4}{*}{ BPNV } & \multirow[t]{4}{*}{ Sigmoid } & \multicolumn{2}{|c|}{1.2 .4} & $1^{5 t} 104^{\text {th }}$ \\
\hline & 961 & 2 & 1 & & & \multicolumn{2}{|l|}{$3,5,7$} & $2^{\text {nd }}$ to $5^{\text {th }}$ \\
\hline & 81 & 4 & 1 & & & \multicolumn{2}{|c|}{1.2 .3} & $1^{\text {st }}$ to $2^{\text {nd }}$ \\
\hline & 300 & 15 & 1 & & & \multicolumn{2}{|c|}{$2,4,6,8,10$} & $1^{\text {st }}$ to $2^{\text {nd }}$ \\
\hline $\begin{array}{l}\text { Madavan et al. } \\
{[31]}\end{array}$ & - & 13 & 1 & $\begin{array}{l}\text { BPNN } \\
\text { (3-layer) }\end{array}$ & Sigmoid & \multicolumn{2}{|c|}{$15 \& 7$} & $1^{\text {st }}$ to $2^{\text {nd }}$ \\
\hline $\begin{array}{l}\text { Nikolaidis et } \\
\text { al. [39] }\end{array}$ & 400 & 50 & 1 & BPNN & Sigmoid & \multicolumn{2}{|c|}{$\begin{array}{l}\sigma \text { of } N N \text { is insensitive } \\
\text { to no of neurons }\end{array}$} & $2^{\text {ndd }}$ \\
\hline \multirow{5}{*}{$\begin{array}{l}\text { Papila et al. } \\
{[49]}\end{array}$} & 9 & 2 & 1 & \multirow{5}{*}{$\begin{array}{l}\text { RBNY } \\
\& \\
\text { BPVY }\end{array}$} & \multirow{5}{*}{$\begin{array}{l}\text { radbas } \\
\& \\
\text { Sigmoid }\end{array}$} & 8,9 & 4 & $2^{\text {nd }}$ to $5^{\text {th }}$ \\
\hline & 15 & 2 & 1 & & & 12,15 & + & $2^{\text {nd }}$ to $5^{\text {h }}$ \\
\hline & 25 & 2 & 1 & & & 20.25 & 4 & $2^{\text {nd }}$ to $5^{1 / h}$ \\
\hline & 255 & 2 & 1 & & & 253,255 & - & $2^{\text {ndd }}$ to $4^{\text {th }}$ \\
\hline & 765 & 2 & 1 & & & 765 & - & - \\
\hline \multirow{3}{*}{$\begin{array}{l}\text { Rai and Madavan } \\
{[52]}\end{array}$} & $3 \& 5$ & 1 & 1 & \multirow{3}{*}{$\begin{array}{l}\text { BPNV } \\
\text { (3- layer) }\end{array}$} & \multirow[t]{3}{*}{ Sigmoid } & \multicolumn{2}{|l|}{$1 \& 2$} & $1^{\text {st }}$ to $2^{\text {ind }}$ \\
\hline & 27 & 3 & 1 & & & \multicolumn{2}{|l|}{$7 \& 3$} & $1^{\text {st }} 102^{\text {nd }}$ \\
\hline & - & 15 & 1 & & & \multicolumn{2}{|l|}{-} & - \\
\hline Shyy et al.[57] & 45 & 3 & 2 & RBNN & radbas & 42 and 45 & & $2^{\text {nd }}$ to $3^{\text {nd }}$ \\
\hline \multirow{2}{*}{$\begin{array}{l}\text { Vaidyanathan et } \\
\text { al. [71] }\end{array}$} & 45 & 3 & 2 & \multirow[t]{2}{*}{ RBNN } & \multirow[t]{2}{*}{ radbas } & \multirow{2}{*}{\multicolumn{2}{|c|}{42 and +5}} & \multirow[t]{2}{*}{$2^{\text {nd }}$ to $3^{\text {rd }}$} \\
\hline & 76 & 6 & 2 & & & & & \\
\hline
\end{tabular}


Table 3. Design variables and design space tor single-, 2- and 3-Stage turbines

(All geometric design variables are normalized by the baseline values)

\begin{tabular}{|c|c|c|c|c|c|c|c|c|}
\hline \multicolumn{3}{|c|}{ SINGLE-STAGE } & \multicolumn{3}{|c|}{ 2-STAGE } & \multicolumn{3}{|c|}{ 3-STAGE } \\
\hline Variable & $\begin{array}{l}\text { Lower } \\
\text { Limit }\end{array}$ & $\begin{array}{l}\text { Upper } \\
\text { Limit }\end{array}$ & Variable & $\begin{array}{c}\text { Lower } \\
\text { Limit }\end{array}$ & $\begin{array}{l}\text { Upper } \\
\text { Limit }\end{array}$ & Variable & $\begin{array}{c}\text { Lower } \\
\text { Limit } \\
\end{array}$ & $\begin{array}{l}\text { Upper } \\
\text { Limit } \\
\end{array}$ \\
\hline $\begin{array}{l}\text { Mean } \\
\text { Diameter, D }\end{array}$ & 0.50 & 1.50 & $\begin{array}{l}\text { Mean } \\
\text { Diameter, D }\end{array}$ & 0.50 & 1.50 & $\begin{array}{l}\text { Mean } \\
\text { Diameter, D }\end{array}$ & 0.50 & 1.50 \\
\hline Speed, RPM & 0.70 & 1.30 & Speed, $R P M$ & 0.70 & 1.30 & Speed, $R P M$ & 0.70 & 1.30 \\
\hline $\begin{array}{l}\text { Blade } \\
\text { Annulus } \\
\text { Area, } A_{\text {ann }} \\
\end{array}$ & 0.70 & 1.30 & $\begin{array}{l}\text { Blade } \\
\text { Annulus } \\
\text { Area, A Ann } \\
\end{array}$ & 0.70 & 1.30 & $\begin{array}{l}\text { Blade } \\
\text { Annulus } \\
\text { Area, } A_{\text {ann }} \\
\end{array}$ & 0.70 & 1.30 \\
\hline $\begin{array}{l}\text { Vane Axial } \\
\text { Chord, } c_{v}\end{array}$ & 0.39 & 1.71 & $\begin{array}{l}1^{\text {st }} \text { Blade } \\
\text { Height ( } \% \text { of } \\
\text { Exit Blade), } \\
h_{1}\end{array}$ & 0.90 & 1.50 & $\begin{array}{l}1^{\text {st }} \text { Blade } \\
\text { Height (\% } \\
\text { of Exit } \\
\text { Blade), } h_{1}\end{array}$ & 0.90 & 1.50 \\
\hline $\begin{array}{l}\text { Blade Axial } \\
\text { Chord, } c_{b}\end{array}$ & 0.26 & 1.14 & $\begin{array}{l}\mathrm{l}^{\text {st }} \text { Vane } \\
\text { Axial Chord, } \\
\mathrm{c}_{\mathrm{vl}}\end{array}$ & 0.39 & 1.71 & $\begin{array}{l}1^{\text {st }} \text { Vane } \\
\text { Axial } \\
\text { Chord, } c_{v 1}\end{array}$ & 0.39 & 1.71 \\
\hline $\begin{array}{l}\text { Stage } \\
\text { Reaction, sr }\end{array}$ & $0.0 \%$ & $50 \%$ & $\begin{array}{l}1^{\text {st }} \text { Blade } \\
\text { Axial Chord, } \\
c_{b l}\end{array}$ & 0.26 & 1.14 & $\begin{array}{l}1^{\text {st }} \text { Blade } \\
\text { Axial } \\
\text { Chord, } c_{b 1}\end{array}$ & 0.26 & 1.14 \\
\hline & & & $\begin{array}{l}2^{\text {nd }} \text { Vane } \\
\text { Axial Chord, } \\
c,\end{array}$ & 0.21 & 1.41 & $\begin{array}{l}2^{\text {tid }} \text { Vane } \\
\text { Axial } \\
\text { Chord, } c_{\mathrm{v} 2}\end{array}$ & 0.21 & 1.41 \\
\hline & & & $\begin{array}{l}2^{\text {nd }} \text { Blade } \\
\text { Axial Chord. } \\
c_{b 2}\end{array}$ & 0.17 & 1.13 & $\begin{array}{l}2^{\text {nd }} \text { Blade } \\
\text { Axial } \\
\text { Chord, } c_{b 2}\end{array}$ & 0.17 & 1.13 \\
\hline & & & $\begin{array}{l}1^{\text {st }} \text { Stage } \\
\text { Reaction. } \mathrm{sr}_{\mathrm{i}}\end{array}$ & $0.0^{\circ}$ & $50 \%$ & $\begin{array}{l}3^{\text {rd }} \text { Vane } \\
\text { Axial } \\
\text { Chord, } c_{\mathrm{y} 3}\end{array}$ & 0.21 & 1.41 \\
\hline & & & $\begin{array}{l}2^{\text {nd }} \text { Stage } \\
\text { Reaction. } \mathrm{Sr}_{2}\end{array}$ & $0.0 \%$ & $50 \%$ & $\begin{array}{l}3^{\text {rd }} \text { Blade } \\
\text { Axial } \\
\text { Chord, } c_{b 3}\end{array}$ & 0.17 & 1.13 \\
\hline & & & $\begin{array}{l}1^{\text {st }} \text { Work } \\
\text { Fraction, wf }\end{array}$ & $50^{\sigma_{C}}$ & $85 \%$ & $\begin{array}{l}1^{\text {si }} \text { Stage } \\
\text { Reaction, } \mathrm{sr}_{1}\end{array}$ & $0.0 \%$ & $50 \%$ \\
\hline & & & & & & $\begin{array}{l}2^{\text {nd }} \text { Stage } \\
\text { Reaction, } \mathrm{sr}_{2}\end{array}$ & $0.0 \%$ & $50 \%$ \\
\hline & & & & & & $\begin{array}{l}3^{\pi} \text { Stage } \\
\text { Reaction, } \mathrm{sr}_{3}\end{array}$ & $0.0 \%$ & $50 \%$ \\
\hline & & & & & & $\begin{array}{l}1^{\text {st }} \text { Work } \\
\text { Fraction, wi }\end{array}$ & $40 \%$ & $80 \%$ \\
\hline & & & & & & $\begin{array}{l}2^{\text {ld }} \text { Work } \\
\text { Fraction, wt }\end{array}$ & $30 \%$ & $10 \%$ \\
\hline
\end{tabular}


Table 4. Different cubic polynomials for $E R E$. (Dependent variables: $V_{o}$ and $L_{r o m b}, 15$ training points, 10

\begin{tabular}{|c|c|c|c|c|c|}
\hline $\begin{array}{c}\text { Model } \\
\#\end{array}$ & Coefficient $=0$ & $\begin{array}{c}\text { Terms } \\
\text { Removed }\end{array}$ & Terms Included & $\overline{\sigma_{a}}(\%)$ & $\sigma(\%)$ \\
\hline 1 & $V / V_{o}^{3}$ & & & 0.09 & 0.21 \\
\hline 2 & $V / V o^{3}$ & $V / N o^{2} L_{r o m b}$ & & 0.08 & 0.21 \\
\hline 3 & & $V \mathrm{No}^{2} L_{\text {comb. }} V, \mathrm{Vo}^{3}$ & & 0.08 & 0.21 \\
\hline 4 & & $V / N o^{2} L_{\text {comb. }} V N^{\prime}{ }^{J}$ & $L_{\text {comb }}{ }^{+}$ & 0.09 & 0.21 \\
\hline 5 & & $V / N o^{2} L_{c o m b}, V, N o$ & $L_{c u m b}{ }^{*}, V / V_{0}{ }^{2} L_{c o m s}{ }^{2}$ & 0.09 & 0.21 \\
\hline$\frac{5}{6}$ & & $V / N o^{2} L_{\text {comb }} V N^{3}$ & $\begin{array}{c}L_{c o m b}, V / V_{n}{ }^{2} L_{c o m b}{ }^{2} \\
V N o L_{c o m b}{ }^{3}\end{array}$ & 0.10 & 0.21 \\
\hline
\end{tabular}

Table 5. Different cubic polynomials for $Q$. (Dependent variables: $O / F$ and $V / V_{o}, 9$ training points, 4 test

\begin{tabular}{|c|c|c|c|c|c|}
\hline $\begin{array}{c}\text { Model } \\
\#\end{array}$ & Coefficient $=0$ & $\begin{array}{c}\text { Terms } \\
\text { Removed }\end{array}$ & Terms Included & $\sigma_{a}(\%)$ & $\sigma(\%)$ \\
\hline 1 & $V / N o^{3}, O / F^{j}$ & & & 5.58 & 2.23 \\
\hline 2 & $O / F^{3}$ & $V / \mathrm{No}^{2}$ & & 5.58 & 2.09 \\
\hline 3 & & $V / N^{-}, O / F^{3}$ & & 5.58 & 2.09 \\
\hline 4 & & $V / N^{\circ}, O / F^{3}$ & & 5.58 & 2.23 \\
\hline 5 & & $V A o^{3}, O / F^{3}, V / o^{2}$ & & 3.96 & 2.09 \\
\hline 6 & & $V A \mathrm{Vo}^{3}, \mathrm{O} / \mathrm{F}^{3}, \mathrm{~V}_{\mathrm{N}} \mathrm{Vo}^{2}$ & $V / N o^{2} O / F^{2}$ & 5.58 & 2.09 \\
\hline
\end{tabular}

Table 6. Neural Network architectures used to design the model for shear co-axial injector element. ( $s c=$

\begin{tabular}{|l|l|l|l|l|l|l|l|}
\hline Scheme & $\begin{array}{l}\text { \# of } \\
\text { Layers }\end{array}$ & $\begin{array}{l}\text { \# of neurons in } \\
\text { the } \\
\text { layer }\end{array}$ & $\begin{array}{l}\text { \# of neurons in } \\
\text { the output layer }\end{array}$ & Error goal aimed for during training \\
& & $E R E$ & $Q$ & $E R E$ & $Q$ & $E R E$ & $Q$ \\
\hline $\begin{array}{l}\text { RBNN } \\
\text { (Solverbe) }\end{array}$ & 2 & 15 & 9 & 1 & 1 & $0.0\{s c=3.25\}$ & $0.0\{s c=1.20\}$ \\
\hline RBNN (Solverb) & 2 & 14 & 8 & 1 & 1 & $0.001\{s c=1.05\}$ & $0.001\{s c=1.05\}$ \\
\hline BPNN & 2 & 8 & 4 & 1 & 1 & 0.01 & 0.01 \\
\hline
\end{tabular}

Table 7. RMS error in predicting the values of the objective function by various schemes for the shear co-

\begin{tabular}{|l|c|c|}
\hline Scheme & $\boldsymbol{\sigma}$ for $\boldsymbol{E R E}(\%)$ & $\boldsymbol{\sigma}$ for $Q(\%)$ \\
\hline RBNN (Solverbe) & 0.20 & 1.40 \\
\hline RBNN (Solverb) & 0.13 & 1.53 \\
\hline BPNN & 0.18 & 0.83 \\
\hline Partial Cubic RS & 0.21 & 2.23 \\
\hline Quadratic RS & 0.28 & 3.49 \\
\hline
\end{tabular}


Table 8. Optimum values obtained with cubic and quadratic for case 1 . (Constraints: $4 \leq \mathrm{O} / \mathrm{F} \leq 6,4 \leq$ $V_{f} / V_{0} \leq 6$, and $\left.L_{\text {comb }} \leq 7\right)$ (Values in the parenthesis are the exact response of the injector model

\begin{tabular}{|c|c|c|c|c|c|c|c|c|c|c|c|}
\hline & & \multicolumn{5}{|c|}{ Cubic } & \multicolumn{5}{|c|}{ Quadratic } \\
\hline $\begin{array}{l}W_{\text {ERE }} \\
(\mathrm{s})\end{array}$ & $\begin{array}{l}W_{Q} \\
(t)\end{array}$ & $\mathrm{O} / \mathrm{F}$ & $\bar{V}_{f} / V_{0}$ & $\bar{L}_{\text {romb }}$ & ERE & $Q$ & $\mathrm{O} / \mathrm{F}$ & $V_{0} / V_{0}$ & Lamb & ERE & $\mathrm{Q}$ \\
\hline 1 & 10 & 6.0 & 5.41 & 7.0 & $\begin{array}{l}99.02 \\
199.001\end{array}$ & $\begin{array}{l}0.664 \\
(0.654)\end{array}$ & 6.0 & 6.00 & 7.0 & $\begin{array}{l}99.17 \\
(99.20) \\
\end{array}$ & $\begin{array}{l}0.669 \\
(0.642) \\
\end{array}$ \\
\hline 1 & 1 & 6.0 & 6.00 & 7.0 & 99.15 & 0.669 & 6.0 & 6.00 & 7.0 & 99.17 & 0.669 \\
\hline 10 & I & 6.0 & 6.00 & 7.0 & 99.15 & 0.669 & 6.0 & 6.00 & 7.0 & 99.17 & 0.669 \\
\hline
\end{tabular}

Table 9. Optimum values obtained with cubic and quadratic for case 2 . (Constraints: $4 \leq \mathrm{O} / \mathrm{F} \leq 6,5 \leq$ $V_{f} / V_{0} \leq 7$, and $L_{\text {comb }} \leq 7$ ) (Values in the parenthesis are the exact response of the injector model \}

\begin{tabular}{|c|c|c|c|c|c|c|c|c|c|c|c|}
\hline & & Cubic & & & & & Quad & & & & \\
\hline $\begin{array}{l}W_{\text {ERE }} \\
\text { (s) }\end{array}$ & $\begin{array}{l}W_{Q} \\
(t)\end{array}$ & $\mathrm{O} / \mathrm{F}$ & $\overline{V_{0}} \mathbf{V}_{0}$ & $\bar{L}$ Lomb & ERE & $\bar{Q}$ & $\mathrm{O} / \mathrm{F}$ & $V_{f} / V_{0}$ & $L_{\text {comb }}$ & ERE & $Q$ \\
\hline 1 & 10 & 6.0 & 5.41 & 7.0 & $\begin{array}{l}99.02 \\
(99.00)\end{array}$ & $\begin{array}{l}0.664 \\
(0.654)\end{array}$ & 6.0 & 6.52 & 7.0 & $\begin{array}{l}99.31 \\
(99.10)\end{array}$ & $\begin{array}{l}0.684 \\
(0.716) \\
\end{array}$ \\
\hline 1 & 1 & 6.0 & 6.34 & 7.0 & $\begin{array}{l}99.21 \\
(99.20)\end{array}$ & $\begin{array}{l}0.674 \\
(0.691)\end{array}$ & 6.0 & 7.00 & 7.0 & $\begin{array}{l}99.42 \\
(99.30)\end{array}$ & $\begin{array}{l}0.702 \\
(0.728) \\
\end{array}$ \\
\hline 10 & 1 & 6.0 & 7.00 & 7.0 & 99.32 & 0.690 & 6.0 & 7.00 & 7.0 & 99.42 & 0.702 \\
\hline
\end{tabular}

Table 10. Optimum values obtained with cubic and quadratic for case 3 . (Constraints: $4 \leq \mathrm{O} / \mathrm{F} \leq 6,6 \leq$ $\mathrm{V}_{\mathrm{r}}, \mathrm{V}_{\mathrm{o}} \leq 8$. and $\mathrm{L}_{\mathrm{xomb}} \leq 7$ )

\begin{tabular}{|c|c|c|c|c|c|c|c|c|c|c|c|}
\hline & & \multicolumn{5}{|c|}{ Cubic } & \multicolumn{5}{|c|}{ Quadratic } \\
\hline $\begin{array}{l}W_{\text {ERE }} \\
\text { (s) }\end{array}$ & $\begin{array}{l}W_{Q} \\
\text { (t) }\end{array}$ & $\mathrm{O} / \mathrm{F}$ & $\mathrm{V}_{0} \mathrm{~V}_{0}$ & Lomb & ERE & $Q$ & $\mathrm{O} / \mathrm{F}$ & $\mathbf{V}_{\mathrm{f}} / \mathbf{V}_{\mathrm{o}}$ & Lomb & ERE & $\mathrm{Q}$ \\
\hline 1 & 10 & 6.0 & 6.00 & 7.0 & 99.15 & 0.669 & 6.0 & 6.52 & 7.0 & 99.31 & 0.684 \\
\hline 1 & 1 & 6.0 & 6.34 & 7.0 & 99.21 & 0.674 & 6.0 & 8.00 & 7.0 & 99.67 & 0.753 \\
\hline 10 & 1 & 6.0 & 8.00 & 7.0 & 99.42 & 0.728 & 6.0 & 8.00 & 7.0 & 99.67 & 0.753 \\
\hline
\end{tabular}


Table 11. The RMS error in the prediction of $E R E$ and $Q$ for different values of spread constant. The error goal used for Solverb is 0.001

\begin{tabular}{|c|c|c|c|c|c|}
\hline & Solverbe & Solverbe & Solverb & Solverb & Solverb \\
\hline$s c$ & $\begin{array}{l}\text { RMS error } \\
\text { (ERE) }\end{array}$ & $\begin{array}{l}\text { RMS error } \\
\text { (Q) }\end{array}$ & $\begin{array}{l}\text { RMS error } \\
\text { (ERE) }\end{array}$ & $\begin{array}{l}\text { RMS error } \\
\text { (Q) }\end{array}$ & No of neurons \\
\hline 0.50 & 1.493 & 0.179 & 1.733 & 0.287 & 44 \\
\hline 0.75 & 0.745 & 0.135 & 0.675 & 0.135 & 44 \\
\hline 1.00 & 0.152 & 0.022 & 0.153 & 0.017 & 42 \\
\hline 1.05 & 0.190 & 0.011 & 0.128 & 0.012 & 44 \\
\hline 1.25 & 0.316 & 0.010 & 0.267 & 0.022 & 44 \\
\hline 1.50 & 0.336 & 0.022 & 0.309 & 0.030 & 44 \\
\hline 1.75 & 0.369 & 0.022 & 0.310 & 0.021 & 44 \\
\hline 2.00 & 0.308 & 0.016 & 0.296 & 0.019 & 41 \\
\hline 2.25 & 0.279 & 0.020 & 1.846 & 0.045 & 43 \\
\hline 2.50 & 0.325 & 0.017 & 0.744 & 0.025 & 43 \\
\hline
\end{tabular}

Table 12. Optimum values obtained with cubic and quadratic for case 1 (enhanced data set). (Constraints: $4 \leq \mathrm{O} / \mathrm{F} \leq 6,4 \leq \mathrm{V}_{\uparrow} / \mathrm{V}_{0} \leq 6$, and $\mathrm{L}_{\mathrm{romb}} \leq 7$ ) $\{$ Compare with Table 8$\}$

\begin{tabular}{|c|c|c|c|c|c|c|c|c|c|c|c|}
\hline & & \multicolumn{5}{|c|}{ Cubic } & \multicolumn{5}{|c|}{ Quadratic } \\
\hline $\begin{array}{l}W_{\text {ERE }} \\
\text { (s) }\end{array}$ & $\begin{array}{l}W_{Q} \\
(t)\end{array}$ & $\mathrm{O} / \mathrm{F}$ & $V_{\mathrm{P}} / \mathrm{V}_{\mathrm{o}}$ & Lomb & ERE & $\mathrm{Q}$ & $\mathrm{O} / \mathrm{F}$ & $V_{t} / V_{0}$ & $\mathrm{~L}_{\text {conno }}$ & ERE & $Q$ \\
\hline 1 & 10 & 6.0 & 5.54 & 7.0 & $\begin{array}{l}99.02 \\
(98.90) \\
\end{array}$ & $\begin{array}{l}0.654 \\
(0.658) \\
\end{array}$ & 6.0 & 5.01 & 7.0 & $\begin{array}{l}98.96 \\
(98.70) \\
\end{array}$ & $\begin{array}{l}0.644 \\
(0.664)\end{array}$ \\
\hline 1 & 1 & 6.0 & 6.00 & 7.0 & 99.12 & 0.658 & 6.0 & 6.00 & 7.0 & 99.25 & 0.658 \\
\hline 10 & 1 & 6.0 & 6.00 & 7.0 & 99.12 & 0.658 & 6.0 & 6.00 & 7.0 & 99.25 & 0.658 \\
\hline
\end{tabular}

Table 13. Optimum values obtained with cubic and quadratic for case 2 (enhanced data set). (Constraints:

\begin{tabular}{|c|c|c|c|c|c|c|c|c|c|c|c|}
\hline & & \multicolumn{5}{|c|}{ Cubic } & \multicolumn{5}{|c|}{ Quadratic } \\
\hline $\begin{array}{l}W_{\text {LRE }} \\
\text { (s) }\end{array}$ & $\begin{array}{l}W_{Q} \\
(t)\end{array}$ & $\mathrm{O} / \mathrm{F}$ & $V_{p} / V_{0}$ & Lomb & ERE & $\mathrm{Q}$ & $\mathrm{O} / \mathrm{F}$ & $V_{1} / V_{0}$ & Lroulb & $\overline{E R E}$ & $Q$ \\
\hline 1 & 10 & 6.0 & 5.54 & 7.0 & $\begin{array}{l}99.02 \\
(98.90)\end{array}$ & $\begin{array}{l}0.654 \\
(0.658)\end{array}$ & 6.0 & 5.01 & 7.0 & $\begin{array}{l}98.96 \\
(98.70)\end{array}$ & $\begin{array}{l}0.644 \\
(0.664)\end{array}$ \\
\hline 1 & 1 & 6.0 & 6.33 & 7.0 & $\begin{array}{l}99.18 \\
(99.10)\end{array}$ & $\begin{array}{l}0.663 \\
(0.666)\end{array}$ & 6.0 & 6.04 & 7.0 & $\begin{array}{l}99.26 \\
(99.20)\end{array}$ & $\begin{array}{l}0.659 \\
(0.642)\end{array}$ \\
\hline 10 & 1 & 6.0 & 7.00 & 7.0 & 99.30 & 0.681 & 6.0 & 7.00 & 7.0 & 99.46 & 0.693 \\
\hline
\end{tabular}


Table 14. Optimum values obtained with cubic and quadratic for case 3 (enhanced data set). (Constraints: $4 \leq \mathrm{O} / \mathrm{F} \leq 6,6 \leq V_{*} V_{0} \leq 8$, and $L_{c o m b} \leq 7$ ) \{Compare with Table 10$\}$

\begin{tabular}{|l|l|l|l|l|l|l|l|l|l|l|l|}
\hline & \multicolumn{9}{|c|}{ Cubic } & \multicolumn{1}{|l|}{ Quadratic } \\
\hline $\begin{array}{l}\mathrm{W}_{\text {ERE }} \\
(\mathrm{s})\end{array}$ & $\begin{array}{l}\mathrm{W}_{\mathrm{Q}} \\
(\mathrm{t})\end{array}$ & $\mathrm{O} / \mathrm{F}$ & $\mathrm{V}_{f} / \mathrm{V}_{\mathrm{o}}$ & $\mathrm{L}_{\text {comb }}$ & ERE & $\mathrm{Q}$ & $\mathrm{O} / \mathrm{F}$ & $\mathrm{V}_{\mathrm{f}} / \mathrm{V}_{\circ}$ & L $_{\text {cont }}$ & ERE & $\mathrm{Q}$ \\
\hline $\mathrm{l}$ & 10 & 6.0 & 6.00 & 7.0 & 99.12 & 0.658 & 6.0 & 6.00 & 7.0 & 99.25 & 0.658 \\
\hline 1 & 1 & 6.0 & $\mathbf{6 . 3 3}$ & 7.0 & 99.19 & 0.663 & 6.0 & $\mathbf{6 . 0 4}$ & 7.0 & 99.26 & 0.659 \\
\hline 10 & 1 & 6.0 & 8.00 & 7.0 & 99.42 & 0.725 & 6.0 & 7.95 & 7.0 & 99.57 & 0.746 \\
\hline
\end{tabular}

Table 15. Optimal Solutions for fixed values of $V_{j} N_{o}$ and given range of $O / F$ and $L_{c r m b}$ obtained with NN and RSM schemes for the shear co-axial injector element. (Constraints: $4 \leq O / F \leq 8,4 \leq L_{\text {iumb }} \leq 7$ ) (error

\begin{tabular}{|c|c|c|c|c|c|}
\hline$V / V_{o}$ & Scheme & $O / F$ & $L_{c a-b}$, in. & $E R E, \%$ & $Q, \mathrm{Btw} / \mathrm{in}^{2}-\mathrm{sec}$ \\
\hline \multirow[t]{7}{*}{4} & RBNN (Solverbe) & 8.0 & 7.0 & $98.60(0.00)$ & $0.588(0.00)$ \\
\hline & RBNN (Solverb) & 8.0 & 7.0 & $98.60(0.00)$ & $0.588(0.00)$ \\
\hline & BPNN & 8.0 & 6.9 & $98.64(0.14)$ & $0.578(1.70)$ \\
\hline & Partial Cubic RS & 8.0 & 7.0 & $98.61(0.01)$ & $0.595(1.19)$ \\
\hline & Quadratic RS & 8.0 & 7.0 & $98.67(0.07)$ & $0.591(0.51)$ \\
\hline & Model & 8.0 & 70 & 98.60 & 0.588 \\
\hline & Model & 8.0 & 6.9 & 98.50 & 0.588 \\
\hline \multirow[t]{6}{*}{6} & RBNN (Solverbe) & 8.0 & 7.0 & $99.20(0.00)$ & $0.512(0.00)$ \\
\hline & RBNN (Solverb) & 8.0 & 7.0 & $99.20(0.00)$ & $0.512(0.00)$ \\
\hline & BPNN & 8.0 & 7.0 & $99.18(0.02)$ & $0.513(0.20)$ \\
\hline & Partial Cubic RS & 8.0 & 7.0 & $99.15(0.05)$ & $0.499(2.54)$ \\
\hline & Quadratic RS & 8.0 & 7.0 & $99.17(0.03)$ & $0.531(3.71)$ \\
\hline & Model & 8.0 & 7.0 & 99.20 & 0.512 \\
\hline \multirow[t]{6}{*}{8} & RBNN (Solverbe) & 8.0 & 7.0 & $99.40(0.00)$ & $0.493(0.00)$ \\
\hline & RBNN (Solverb) & 8.0 & 7.0 & $99.40(0.00)$ & $0.493(0.00)$ \\
\hline & BPNN & 8.0 & 7.0 & $99.41(0.01)$ & $0.500(1.42)$ \\
\hline & Partial Cubic RS & 8.0 & 7.0 & $99.42(0.02)$ & $0.500(1.42)$ \\
\hline & Quadratic RS & 8.0 & 7.0 & $99.67(0.27)$ & $0.471(4.46)$ \\
\hline & Model & 8.0 & 7.0 & 99.40 & 0.493 \\
\hline
\end{tabular}


Table 16. Effect of each variable on the optimization of impingement co-axial injector element -optimal designs for original constraints \& equal weights

\begin{tabular}{|c|c|c|c|c|c|}
\hline $\begin{array}{l}\text { Independent } \\
\text { Variable }\end{array}$ & Constraints & Results Case 1 & Results Case 2 & Results Case 3 & Results Case 4 \\
\hline$\Delta \mathbf{P}_{\mathbf{q}}$ & $100-200$ & 183 & 183 & 179 & 100 \\
\hline$\Delta \mathbf{P}_{\ell}$ & $100-200$ & 100 & 132 & 149 & 100 \\
\hline $\mathbf{L}_{\text {comb }}$ & $2-8$ & 8.0 & 8.0 & 6.6 & 6.5 \\
\hline$\alpha$ & $15-50$ & 33.1 & 18.9 & 22.3 & 24.0 \\
\hline $\begin{array}{l}\text { Dependent } \\
\text { Variable }\end{array}$ & $\begin{array}{c}\text { Desirability } \\
\text { Limits }\end{array}$ & ERE \& $Q_{w}$ & $\begin{array}{c}\text { ERE, } \mathbf{Q}_{w}, \\
\mathrm{H}_{\text {impinge }}\end{array}$ & $\begin{array}{c}\text { ERE, } Q_{w}, \\
\mathbf{H}_{\text {impinge }}, W_{\text {rel }}\end{array}$ & $\begin{array}{c}\text { ERE, } Q_{w}, \\
H_{\text {impinze }}, W_{\text {rel }}, \\
C_{\text {rel }}\end{array}$ \\
\hline ERE & $95.0-99.9$ & 99.9 & 98.3 & 98.0 & 98.0 \\
\hline$Q_{w}$ & $0.7-1.3$ & 0.74 & 0.76 & 0.79 & 0.86 \\
\hline$H_{\text {impinge }}$ & $0.2-1.0$ & - & 0.75 & 0.61 & 0.63 \\
\hline$W_{\text {rel }}$ & $0.9-1.2$ & - & - & 1.1 & 1.1 \\
\hline $\mathrm{C}_{\mathrm{rel}}$ & $0.7-1.1$ & - & 二 & - & 0.93 \\
\hline
\end{tabular}

Table 17. Effect of emphasizing \& life \& performance issues on the optimization of impingement co-axial

\begin{tabular}{|c|c|c|c|c|c|c|}
\hline $\begin{array}{c}\text { Independent } \\
\text { Variable }\end{array}$ & Constraints & $\begin{array}{c}\text { Results } \\
\text { Case 1 }\end{array}$ & Constraints & $\begin{array}{c}\text { Results } \\
\text { Case 2 }\end{array}$ & Constraints & $\begin{array}{c}\text { Results } \\
\text { Case 3 }\end{array}$ \\
\hline$\Delta \mathbf{P}_{0}$ & $100-200$ & 100 & $100-200$ & 158 & $100-200$ & 100 \\
\hline$\Delta \mathbf{P}_{\mathbf{r}}$ & $100-200$ & 100 & $100-200$ & 100 & $100-200$ & 137 \\
\hline $\mathbf{L}_{\text {comb }}$ & $2-8$ & 6.5 & $2-8$ & 7.7 & $2-8$ & 5.2 \\
\hline$\alpha$ & $15-50$ & 24.0 & $15-50$ & 15.0 & $15-50$ & 36.0 \\
\hline $\begin{array}{c}\text { Dependent } \\
\text { Variable }\end{array}$ & $\begin{array}{c}\text { Baseline } \\
\text { Variable } \\
\text { Weight }\end{array}$ & 1 & $\begin{array}{c}\text { Life } \\
\text { Variable } \\
\text { Weight }\end{array}$ & & $\begin{array}{c}\text { Thrust/Weight } \\
\text { Variable } \\
\text { Weight }\end{array}$ & \\
\hline ERE & 1 & 98.0 & 1 & 96.7 & 5 & 99.1 \\
\hline $\mathbf{Q}_{w}$ & 1 & 0.86 & 5 & 0.75 & 1 & 0.95 \\
\hline $\mathbf{H}_{\text {impinpe }}$ & 1 & 0.63 & 5 & 0.94 & 1 & 0.32 \\
\hline $\mathbf{W}_{\text {rel }}$ & 1 & 1.10 & 1 & 1.14 & 5 & 1.05 \\
\hline $\mathbf{C}_{\text {rel }}$ & 1 & 0.93 & 1 & 0.97 & 1 & 0.95 \\
\hline
\end{tabular}


Table 18. Effects of realizing the last increments of performance \& weight on the optimization of impingement co-axial injector element -optimum designs for modified constraints and unequal weights

\begin{tabular}{|c|c|c|c|c|c|c|}
\hline $\begin{array}{c}\text { Independent } \\
\text { Variable }\end{array}$ & $\begin{array}{c}\text { Original } \\
\text { Constraints }\end{array}$ & $\begin{array}{c}\text { Results } \\
\text { Case1 }\end{array}$ & $\begin{array}{c}\text { Modified } \\
\Delta P \\
\text { Constraints }\end{array}$ & $\begin{array}{c}\text { Results } \\
\text { Case } 2\end{array}$ & $\begin{array}{c}\text { Modified } \\
\Delta P \\
\text { Constraints }\end{array}$ & $\begin{array}{l}\text { Results } \\
\text { Case } 3\end{array}$ \\
\hline$\Delta \mathbf{P}_{0}$ & $100-200$ & 100 & $150-200$ & 150 & $150-200$ & 150 \\
\hline$\Delta P_{f}$ & $100-200$ & 137 & $150-200$ & 150 & $150-200$ & 200 \\
\hline $\mathbf{L}_{\text {comb }}$ & $2-8$ & 5.2 & $2-8$ & 5.4 & $2-8$ & 4.4 \\
\hline$\alpha$ & $15-50$ & 36.0 & $15-50$ & 35.6 & $15-50$ & 44.8 \\
\hline $\begin{array}{c}\text { Dependent } \\
\text { Variable }\end{array}$ & $\begin{array}{c}\text { Variable } \\
\text { Weight } \\
(5: 1) \\
\end{array}$ & & $\begin{array}{c}\text { Variable } \\
\text { Weight } \\
(5: 1) \\
\end{array}$ & & $\begin{array}{l}\text { Variable } \\
\text { Weight } \\
(100: 1) \\
\end{array}$ & \\
\hline ERE & 5 & 99.1 & 5 & 99.0 & 10 & 99.1 \\
\hline $\mathbf{Q}_{w}$ & 1 & 0.95 & 1 & 0.84 & 0.1 & 0.95 \\
\hline $\mathrm{H}_{\text {impines }}$ & 1 & 0.32 & 1 & 0.31 & 0.1 & 0.21 \\
\hline $\mathbf{W}_{\text {rel }}$ & 5 & 1.05 & 5 & 1.05 & 10 & 1.01 \\
\hline $\mathrm{C}_{\mathrm{rel}}$ & 1 & 0.95 & 1 & 1.00 & 0.1 & 1.07 \\
\hline
\end{tabular}

Table 19. Effect of each variable on the optimization of swirl co-axial injector element -optimal designs

\begin{tabular}{|c|c|c|c|c|c|c|}
\hline $\begin{array}{c}\text { Independent } \\
\text { Variable }\end{array}$ & Constraints & $\begin{array}{c}\text { Results } \\
\text { Case } 1 \\
\end{array}$ & $\begin{array}{c}\text { Results } \\
\text { Case } 2 \\
\end{array}$ & $\begin{array}{l}\text { Results } \\
\text { Case } 3 \\
\end{array}$ & $\begin{array}{c}\text { Results } \\
\text { Case } 4\end{array}$ & $\begin{array}{l}\text { Results } \\
\text { Case } 5 \\
\end{array}$ \\
\hline$\Delta \mathbf{P}_{0}$ & $100-200$ & 200 & 200 & 200 & 200 & 104 \\
\hline$\Delta \mathbf{P}_{\mathrm{f}}$ & $20-200$ & 41 & 41 & 42 & 47 & 20 \\
\hline $\mathbf{L}_{\text {romb }}$ & $2-8$ & 7.2 & 7.2 & 7.6 & 3.2 & 3.4 \\
\hline$\Theta$ & $30-90$ & 81 & 81 & 37 & 47 & 44 \\
\hline $\begin{array}{l}\text { Dependent } \\
\text { Variable }\end{array}$ & $\begin{array}{l}\text { Desirability } \\
\text { Limits }\end{array}$ & $\overline{\text { ERE }}$ & ERE \& $Q_{w}$ & $\begin{array}{c}\text { ERE, } Q_{w}, \\
Q_{\text {inj }}\end{array}$ & $\begin{array}{l}\text { ERE, } Q_{w}, \\
Q_{\text {inj }}, W_{\text {rel }}\end{array}$ & $\begin{array}{c}\text { ERE, } Q_{w}, \\
Q_{\text {inj }}, W_{\text {rel }}, \\
C_{\text {rel }}\end{array}$ \\
\hline ERE & $92.3-99.0$ & 98.5 & 98.5 & 97.2 & 96.0 & 95.7 \\
\hline$Q_{w}$ & $\begin{array}{l}0.596- \\
0.647\end{array}$ & 0.596 & 0.596 & 0.596 & 0.596 & 0.596 \\
\hline$Q_{\text {ini }}$ & $6.95-36.59$ & 26.8 & 26.8 & 9.1 & 12.0 & 10.5 \\
\hline $\mathbf{w}_{\mathrm{rel}}$ & $\begin{array}{l}0.900- \\
1.154 \\
\end{array}$ & 1.13 & 1.13 & 1.14 & 0.97 & 0.98 \\
\hline $\mathrm{C}_{\mathrm{rel}}$ & $0.73-1.42$ & 0.98 & 0.98 & 0.81 & 0.84 & 0.76 \\
\hline
\end{tabular}


Table 20. Effect of emphasizing life and performance issues on the optimization of swirl co-axial injector

\begin{tabular}{|c|c|c|c|c|c|c|}
\hline $\begin{array}{c}\text { Independent } \\
\text { Variable }\end{array}$ & Constraints & $\begin{array}{c}\text { Results } \\
\text { Baseline }\end{array}$ & Constraints & $\begin{array}{c}\text { Results } \\
\text { Case 1 }\end{array}$ & Constraints & $\begin{array}{c}\text { Results } \\
\text { Case 2 }\end{array}$ \\
\hline$\Delta \mathbf{P}_{\mathbf{q}}$ & $100-200$ & 104 & $100-200$ & 200 & $100-200$ & 200 \\
\hline$\Delta \mathbf{P}_{\mathbf{f}}$ & $20-200$ & 20 & $20-200$ & 32 & $20-200$ & 44 \\
\hline $\mathbf{L}_{\text {comb }}$ & $2-8$ & 3.4 & $2-8$ & 3.6 & $2-8$ & 2.9 \\
\hline $\boldsymbol{\theta}$ & $30-90$ & 44.0 & $30-90$ & 30.0 & $30-90$ & 72.0 \\
\hline $\begin{array}{c}\text { Dependent } \\
\text { Variable }\end{array}$ & $\begin{array}{c}\text { Baseline } \\
\text { Variable } \\
\text { Weight }\end{array}$ & 1 & $\begin{array}{c}\text { Life } \\
\text { Variable } \\
\text { Weight }\end{array}$ & & $\begin{array}{c}\text { Thrust/Weight } \\
\text { Variable } \\
\text { Weight }\end{array}$ & \\
\hline $\mathbf{E R E}$ & 1 & 95.7 & 1 & 95.3 & 10 & 96.7 \\
\hline $\mathbf{Q}_{\mathbf{w}}$ & 1 & 0.596 & 5 & 0.596 & 1 & 0.596 \\
\hline $\mathbf{Q}_{\mathbf{w}}$ & 1 & 10.5 & 10 & 6.9 & 1 & 22.6 \\
\hline $\mathbf{W}_{\text {rel }}$ & 1 & 0.98 & 1 & 0.99 & 2 & 0.96 \\
\hline $\mathbf{C}_{\mathrm{rel}}$ & 1 & 0.76 & 1 & 0.79 & 1 & 0.94 \\
\hline
\end{tabular}

Table 21. Optimization summary for 1,2 and 3-stage turbine with response surface in original design

\begin{tabular}{|c|c|c|c|c|c|c|c|}
\hline & 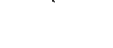 & \multicolumn{3}{|c|}{ Original Design Space } & \multicolumn{3}{|c|}{ Refined Design Space } \\
\hline & & $\eta_{\text {opt }}$ & $W_{o p t}$ & $\Delta$ pay $_{\text {opt }}$ & $\eta_{\text {opt }}$ & $W_{\text {opt }}$ & $\Delta$ pay $_{\text {opt }}$ \\
\hline \multirow{3}{*}{$\Delta$ pay } & 1-stage & 0.77 & 0.73 & -0.21 & 0.77 & 0.73 & -0.21 \\
\hline & 2-stage & 1.10 & 1.05 & 0.11 & 1.13 & 1.04 & 0.15 \\
\hline & 3-stage & 1.24 & 1.62 & 0.14 & 1.20 & 1.54 & 0.11 \\
\hline
\end{tabular}

Table 22. Values of $\sigma_{a}$ and $\sigma$ for different response surfaces of $\eta$ and $W$ for the supersonic turbine.

\begin{tabular}{|l|l|l|l|l|}
\hline Scheme & $\sigma_{\mathbf{a}}$ for $\eta(\boldsymbol{\%})$ & $\sigma$ for $\eta(\%)$ & $\sigma_{\mathrm{a}}$ for $W(\%)$ & $\sigma$ for $W(\%)$ \\
\hline Quadratic RS & 2.51 & 0.90 & 0.82 & 1.27 \\
\hline Reduced Cubic RS & 1.95 & 1.03 & 0.40 & 1.22 \\
\hline
\end{tabular}

Table 23. Neural Network architectures used to design the models for $\eta$ and $W$ of the supersonic turbine.

\begin{tabular}{|l|c|c|c|c|c|c|c|}
\hline Scheme & $\begin{array}{l}\text { \# of } \\
\text { Layers }\end{array}$ & $\begin{array}{l}\text { \# of neurons in the } \\
\text { hidden layer }\end{array}$ & $\begin{array}{l}\text { \# of neurons in the } \\
\text { output layer }\end{array}$ & \multicolumn{2}{c|}{$\begin{array}{l}\text { Error goal aimed for during } \\
\text { training }\end{array}$} \\
\hline & & $\eta$ & $W$ & $\eta$ & $W$ & $\eta$ & $W$ \\
\hline RBNN (Solverbe) & 2 & 76 & 76 & 1 & 1 & 0.0 & 0.0 \\
$\{s c=9.50\}$ & $\{s c=9.45\}$ \\
\hline RBNN (Solverb) & 2 & 37 & 75 & 1 & 1 & $\begin{array}{c}0.001 \\
\{s c=6.50\}\end{array}$ & $\begin{array}{c}0.001 \\
\{c=8.35\}\end{array}$ \\
\hline BPNN & 2 & 5 & 60 & 1 & 1 & 0.001 & 0.001 \\
\hline
\end{tabular}


Table 24. RMS error in predicting the values of $\eta$ and $W$ by various schemes for the supersonic turbine.

\begin{tabular}{|l|c|c|}
\hline Scheme & $\sigma$ for $\eta(\%)$ & $\sigma$ for $\boldsymbol{W}(\%)$ \\
\hline RBNN (Solverbe) & 1.25 & 1.10 \\
\hline RBNN (Solverb) & 0.29 & 1.10 \\
\hline BPNN & 0.78 & 2.56 \\
\hline Reduced Cubic RS & 1.03 & 1.22 \\
\hline
\end{tabular}

Table 25. Optimal solutions with constraints on $V_{\text {puch }}$ and $A N^{2}$ for a supersonic turbine. (error given in parenthesis for each prediction is in $\%)\left(V_{\text {pitch }}=1.148\right.$ and $A N^{2}=1.132$ in all the cases) (All the variables are normalized by their respective baseline values).

\begin{tabular}{|c|c|c|c|c|c|c|c|c|c|}
\hline Scheme & $D$, in. & $R P M$ & $A_{\mathrm{ann}}$, in $^{2}$ & $C_{v}$, in. & $C_{b}$, in. & $K_{r}, \%$ & $\eta$ & $W$, lbs & $\Delta p a y$, lbs \\
\hline $\begin{array}{l}\text { RBNN } \\
\text { (Solverbe) }\end{array}$ & 0.972 & 1.181 & 0.811 & 1.443 & 0.836 & 0.0 & $\begin{array}{l}0.810 \\
(5.80)\end{array}$ & $\begin{array}{l}0.636 \\
(0.74) \\
\end{array}$ & $\begin{array}{l}-0.139 \\
(29.80) \\
\end{array}$ \\
\hline Meanline & 0.972 & 1.181 & 0.811 & 1.443 & 0.836 & 0.0 & 0.766 & 0.641 & -0.197 \\
\hline $\begin{array}{l}\text { RBNN } \\
\text { (Solverb) }\end{array}$ & 0.999 & 1.149 & 0.857 & 1.483 & 0.792 & 0.0 & $\begin{array}{l}0.785 \\
(1.75) \\
\end{array}$ & $\begin{array}{l}0.653 \\
(0.17) \\
\end{array}$ & $\begin{array}{l}-0.177 \\
(9.16) \\
\end{array}$ \\
\hline Meanline & 0.999 & 1.149 & 0.857 & 1.483 & 0.792 & 0.0 & 0.772 & 0.654 & -0.194 \\
\hline BPNN & 1.024 & 1.121 & 0.901 & 1.168 & 1.143 & 0.0 & $\begin{array}{l}0.793 \\
(2.49) \\
\end{array}$ & $\begin{array}{l}0.608 \\
(8.63) \\
\end{array}$ & $\begin{array}{l}-0.153 \\
(21.49)\end{array}$ \\
\hline Meanline & 1.024 & 1.121 & 0.901 & 1.168 & 1.143 & 0.0 & 0.772 & 0.666 & -0.195 \\
\hline $\begin{array}{l}\text { Reduced } \\
\text { Cubic RS }\end{array}$ & 0.903 & 1.272 & 0.700 & 1.706 & 0.871 & 0.0 & $\begin{array}{l}0.758 \\
(1.50) \\
\end{array}$ & $\begin{array}{l}0.591 \\
(2.10) \\
\end{array}$ & $\begin{array}{l}-0.194 \\
(8.40) \\
\end{array}$ \\
\hline Meanline & 0.903 & 1.272 & 0.700 & 1.706 & 0.871 & 0.0 & 0.746 & 0.604 & -0.211 \\
\hline
\end{tabular}

Table 26. The quality of the second-order response surface obtained for $\eta, W$ and 4 pay of 2-stage turbine for 1990-data (face centered criterion) and 249-data (orthogonal arrays) (Mean values of $\eta, W$ and $4 p a y$ are normalized by the baseline values)

\begin{tabular}{llccc}
\hline & & $\eta$ & $W$ & $\Delta$ pay \\
\hline \multirow{5}{*}{1990 -data } & $\mathrm{R}^{2}$ & 0.995 & 0.996 & 0.995 \\
& $\mathrm{Ra}^{2}$ & 0.994 & 0.996 & 0.995 \\
& Rms-error & $1.31 \%$ & $2.56 \%$ & $9.58 \%$ \\
& Mean & 0.78 & 0.86 & -0.24 \\
\hline \multirow{5}{*}{249 -data } & $\mathrm{R}^{2}$ & 0.995 & 0.998 & 0.994 \\
& $\mathrm{Ra}^{2}$ & 0.992 & 0.997 & 0.992 \\
& Rms-error & $2.128 \%$ & $0.826 \%)$ & $20.68 \%$ \\
& Mean & 0.89 & 0.92 & -0.11 \\
\hline
\end{tabular}


Table 27. Testing of the second-order response surface obtained for $\eta$ and $W$ of 2 -stage turbine for 1990 data (FCCD criterion) and 249-data (OA) with 78- test data

\begin{tabular}{|c|c|c|c|}
\hline \# of design points & \# of test data & $\sigma$ for $\eta(\%)$ & $\sigma$ for $W(\%)$ \\
\hline 249 & 78 & 1.65 & 0.96 \\
\hline 1990 & 78 & 1.67 & 1.21 \\
\hline
\end{tabular}

Table 28. Testing the RBNN and second-order polynomial response surface obtained for $\eta$ and $W$ for preliminary design of 2-stage turbine (original design space)

\begin{tabular}{ccccccc}
\hline $\begin{array}{c}\text { Number of } \\
\text { training } \\
\text { data }\end{array}$ & $\begin{array}{c}\text { Number of } \\
\text { test data }\end{array}$ & sc & $\begin{array}{c}\sigma \text { for } \eta(\%) \\
\text { using RBNN } \\
\text { (Solverbe) }\end{array}$ & $\begin{array}{c}\sigma \text { for } \eta(\%) \text { using } \\
\text { Polynomial-based } \\
\text { RSM }\end{array}$ & $\begin{array}{c}\sigma \text { for } W(\%) \\
\text { using RBNN } \\
\text { (Solverbe) }\end{array}$ & $\begin{array}{c}\sigma \text { for } W(\%) \text { using } \\
\text { Polynomial-based } \\
\text { RSM }\end{array}$ \\
\hline 249 & 78 & 4.3 & 1.365 & 1.648 & 1.305 & 0.959 \\
1990 & 78 & 3.2 & 2.263 & 1.672 & 1.557 & 1.214 \\
\hline
\end{tabular}

Table 29. Backward elimination procedure for polynomial-based RSM in five variables.

\begin{tabular}{|c|c|c|c|c|c|}
\hline Terms & Min $\left|\mathrm{t}_{0}\right|$ & No. $\left|\mathrm{t}_{0}\right|<2.0$ & $R^{2}$ & $R_{u}{ }^{2}$ & Comments \\
\hline 21 & 0.05 & 15 & 0.922 & 0.811 & \\
\hline 20 & 0.23 & 14 & 0.922 & 0.823 & Removed $y_{3}{ }^{2}$ \\
\hline 19 & 0.45 & 12 & 0.922 & 0.834 & Removed $y_{4}{ }^{2}$ \\
\hline 18 & 0.53 & 9 & 0.921 & 0.841 & Removed $y_{l} y_{4}$ \\
\hline 17 & 0.97 & 8 & 0.919 & 0.848 & Removed $y_{2} y_{5}$ \\
\hline$* 16$ & 1.22 & 6 & 0.915 & 0.848 & Removed $y_{2}{ }^{2}$ \\
\hline 15 & 1.57 & 5 & 0.909 & 0.844 & Removed $y_{1} y_{3}$ \\
\hline
\end{tabular}

Table 30. Comparison between CFD-solutions and polynomial-based RSM-predictions.

\begin{tabular}{|c|c|c|c|c|c|c|c|}
\hline$Y_{1}$ & $Y_{2}$ & $Y_{3}$ & $Y_{4}$ & $Y_{5}$ & $F$ & $\widehat{F}$ (full) & $\widehat{F}$ (reduced) \\
\hline 0.5 & 0.5 & 0.5 & 0.5 & 0.5 & 0.7171 & 0.7148 & 0.7126 \\
\hline 1.0 & 0.5 & 0.0 & 0.5 & 1.0 & 0.7174 & 0.7210 & 0.7174 \\
\hline 0.25 & 0.75 & 0.25 & 0.75 & 0.25 & 0.7148 & 0.7185 & 0.7162 \\
\hline 0.0 & 0.5 & 1.0 & 0.5 & 0.0 & 0.6943 & 0.7333 & 0.7283 \\
\hline
\end{tabular}


Table 31. Predicted RMS error, $\sigma$, for different polynomial models for 3-D wing model: 9-simulation,

\begin{tabular}{|c|c|c|c|c|}
\hline $\begin{array}{l}\text { Model } \\
\text { No }\end{array}$ & MODEL & $\begin{array}{c}\sigma \text { for } \\
9 \text { data }\end{array}$ & $\begin{array}{c}\sigma \text { for } \\
15 \text { data } \\
\end{array}$ & $\begin{array}{c}\sigma \text { for } \\
25 \text { data }\end{array}$ \\
\hline 1 & $c_{1} \mathrm{AR}^{2}+c_{2} \mathrm{AR}+\mathrm{c}_{3} \mathrm{AR} \mathrm{y}_{\mathrm{C}}+\mathrm{c}_{4} \mathrm{y}_{\mathrm{C}}+\mathrm{c}_{5} \mathrm{y}_{\mathrm{C}}{ }^{2}+\mathrm{c}_{6}$ & 0.8047 & 0.5172 & 0.7800 \\
\hline 2 & $c_{1} A R^{2}+c_{2} A R+c_{3} A R y_{C}+c_{4} y_{C}+c_{5} y_{C}^{2}+c_{6}+c_{7} A R^{3}$ & 0.8047 & 0.5475 & 0.8007 \\
\hline 3 & $c_{1} A R^{2}+c_{2} A R+c_{3} A R y_{C}+c_{4} y_{C}+c_{5} y_{C}{ }^{2}+c_{6}+c_{7} y_{C}{ }^{3}$ & 0.8047 & 0.5172 & 0.5524 \\
\hline 4 & $c_{1} A R^{2}+c_{2} A R+c_{3} A R y_{C}+c_{4} y_{C}+c_{5} y_{C}^{2}+c_{6}+c_{7} A R y_{C}^{2}$ & 0.1162 & 0.0738 & 0.6590 \\
\hline 5 & $c_{1} A R^{2}+c_{2} A R+c_{3} A R y_{C}+c_{4} y_{C}+c_{5} y_{C}^{2}+c_{6}+c_{7} y_{C}^{3}+c_{8} A R y_{C}^{2}$ & - & - & 0.3207 \\
\hline 6 & $\begin{array}{l}c_{1} \mathrm{AR}^{2}+c_{2} \mathrm{AR}+c_{3} \mathrm{ARy}_{\mathrm{C}}+\mathrm{c}_{4} \mathrm{y}_{\mathrm{C}}+\mathrm{c}_{5} \mathrm{yC}_{\mathrm{C}}^{2}+\mathrm{c}_{6} \\
+c_{7} \mathrm{yC}_{\mathrm{C}}^{3}+\mathrm{c}_{8} \mathrm{ARy}_{\mathrm{C}}^{2}+\mathrm{c}_{9} \mathrm{y}_{\mathrm{C}} \mathrm{AR}^{2}\end{array}$ & - & - & 0.3262 \\
\hline 7 & $\begin{array}{l}c_{1} A R^{2}+c_{2} A R+c_{3} A R y_{C}+c_{4} y_{C}+c_{5} y_{C}{ }^{2}+c_{6} \\
+c_{7} y_{C}{ }^{3}+c_{8} A R y^{2}+c_{9} A R^{3}\end{array}$ & $\cdot$ & - & 0.6961 \\
\hline 8 & $\begin{array}{l}c_{1} A R^{2}+c_{2} A R+c_{3} A R y_{C}+c_{4} y_{C}+c_{5} y_{C}{ }^{2}+c_{6} \\
+c_{7} y_{C}{ }^{3}+c_{8} A R y_{C}{ }^{2}+c_{9} y_{C} A R^{2}+c_{10} A R^{3}\end{array}$ & - & - & 0.3350 \\
\hline 9 & $c_{1} A R+c_{2} A R y_{C}+c_{3} y_{C}+c_{4} y_{C}{ }^{2}+c_{5}+c_{6} y_{C}^{3}+c_{7} A R y_{C}{ }^{2}$ & - & - & 0.4248 \\
\hline 10 & $c_{1} A R+c_{2} A R y_{C}+c_{3} y_{C}+c_{4} y_{C}^{2}+c_{5}+c_{6} A R^{2} y_{C}$ & - & - & 0.8044 \\
\hline 11 & $\begin{array}{l}c_{1} A R^{2}+c_{2} A R+c_{3} A R y_{C}+c_{4} y_{C}+c_{9} y_{C}{ }^{2}+c_{6} \\
+c_{7} y_{C}{ }^{3}+c_{8} A R y_{C}{ }^{2}+c_{9} y_{C}{ }^{4}\end{array}$ & - & - & 0.2383 \\
\hline 12 & $\begin{array}{l}c_{1} A R^{2}+c_{2} A R+c_{3} A R y_{C}+c_{4} y_{C}+c_{5} y_{C}{ }^{2}+c_{6} \\
+c_{7} y_{C}{ }^{3}+c_{8} A R y_{C}{ }^{2}+c_{9} y_{C}{ }^{4}+c_{10} A R y_{C}{ }^{3}\end{array}$ & - & - & 0.1073 \\
\hline
\end{tabular}

Table 32. Training history of radial-basis networks with Solverb for 3-D wing madel

\begin{tabular}{|c|c|c|c|c|c|c|}
\hline $\begin{array}{c}\text { NN } \\
\text { No. }\end{array}$ & $\begin{array}{c}\text { \# of } \\
\text { Simulations }\end{array}$ & \# of Neurons & \# of Epochs & $\begin{array}{c}\text { Steady State } \\
\text { Error }\end{array}$ & $\begin{array}{c}\text { Spread } \\
\text { Constant }\end{array}$ & Error Goal \\
\hline 1 & 9 & 8 & 7 & $10^{-16}$ & 1.175 & $10^{-2}$ \\
\hline 2 & 15 & 12 & 11 & $10^{-1}$ & 1.175 & $10^{-2}$ \\
\hline 3 & 25 & 20 & 19 & $10^{-3}$ & 1.175 & $10^{-2}$ \\
\hline
\end{tabular}

Table 33. Training history of back-propagation networks with Trainlm for 3-D wing model

\begin{tabular}{|c|c|c|c|c|c|}
\hline $\begin{array}{c}\text { NN } \\
\text { No. }\end{array}$ & $\begin{array}{c}\text { \# of } \\
\text { Simulations }\end{array}$ & \# of Neurons & \# of Epochs & $\begin{array}{c}\text { Steady State } \\
\text { Error }\end{array}$ & Error Goal \\
\hline 1 & 9 & 4 & 23 & $4.5 \times 10^{-4}$ & $10^{-2}$ \\
\hline 2 & 15 & 4 & 12 & $8.5 \times 10^{-3}$ & $10^{-2}$ \\
\hline 3 & 25 & 4 & 105 & $9.96 \times 10^{-3}$ & $10^{-2}$ \\
\hline
\end{tabular}

